
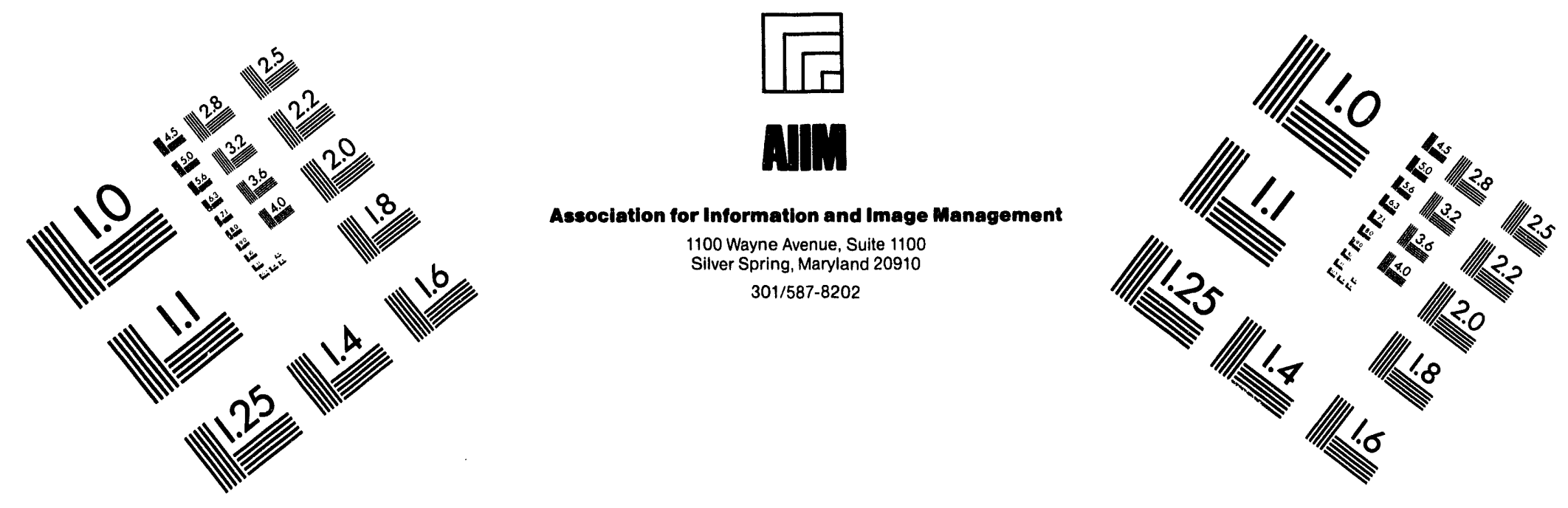

\title{
Centimeter
}

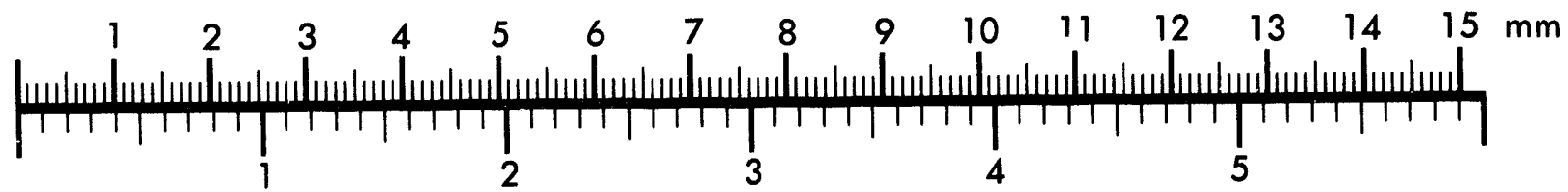

Inches
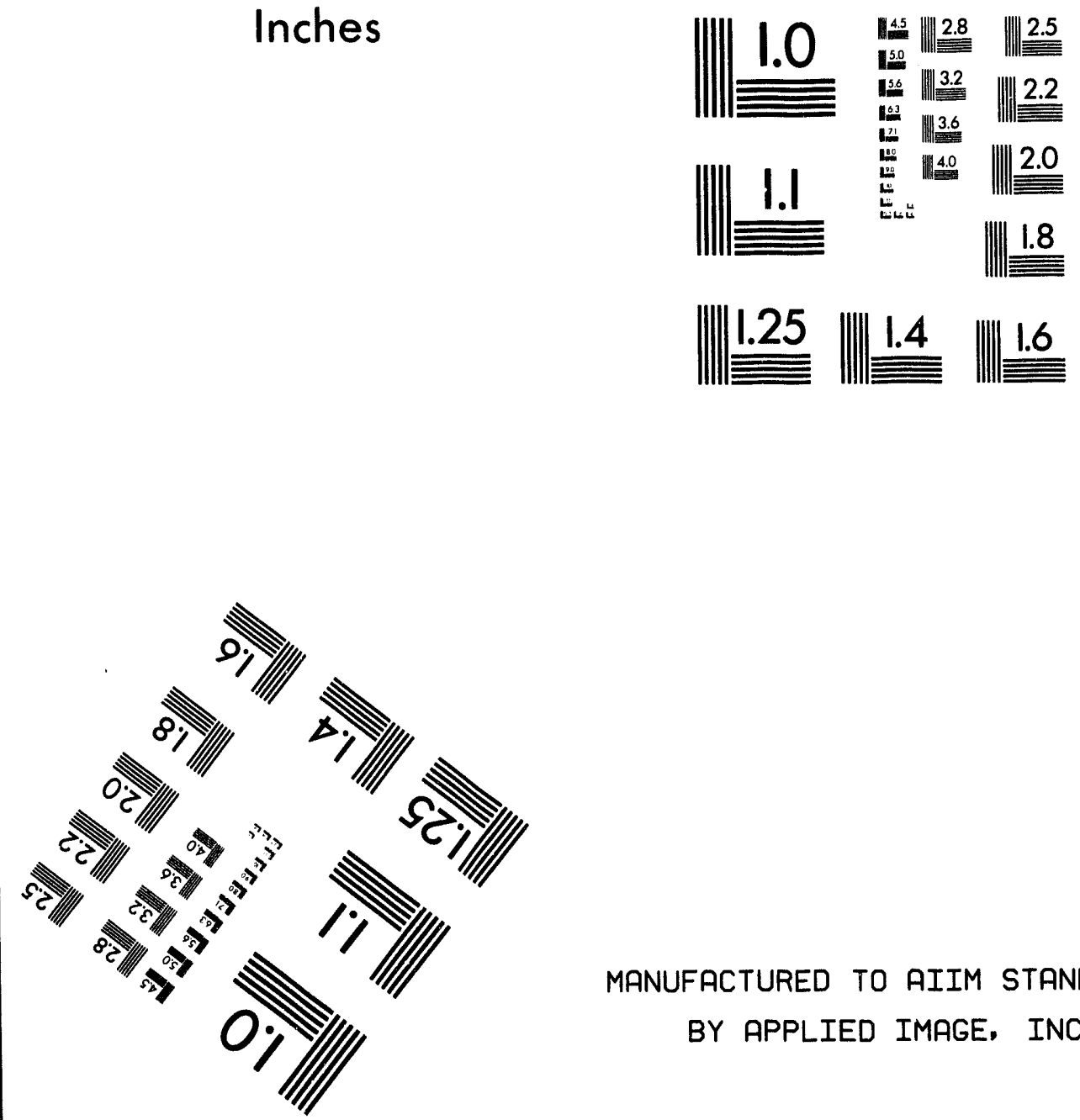

MANUFACTURED TO AIIM STANDARDS

BY APPLIED IMAGE, INC.

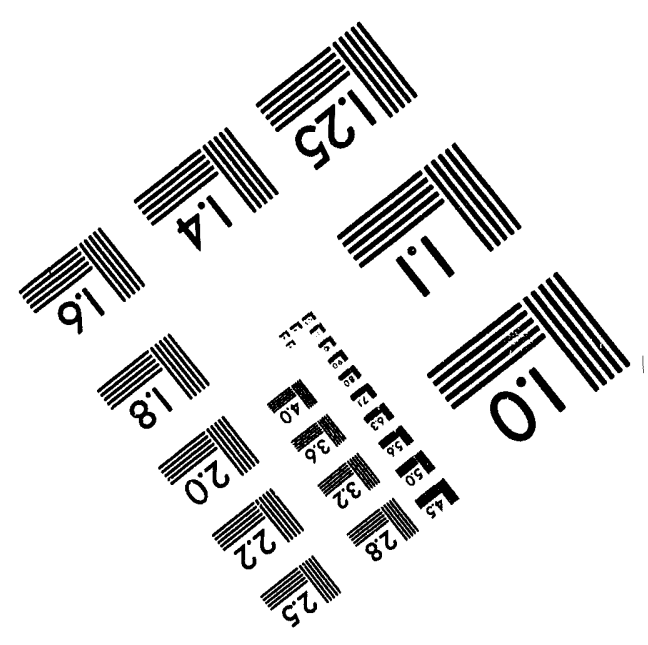



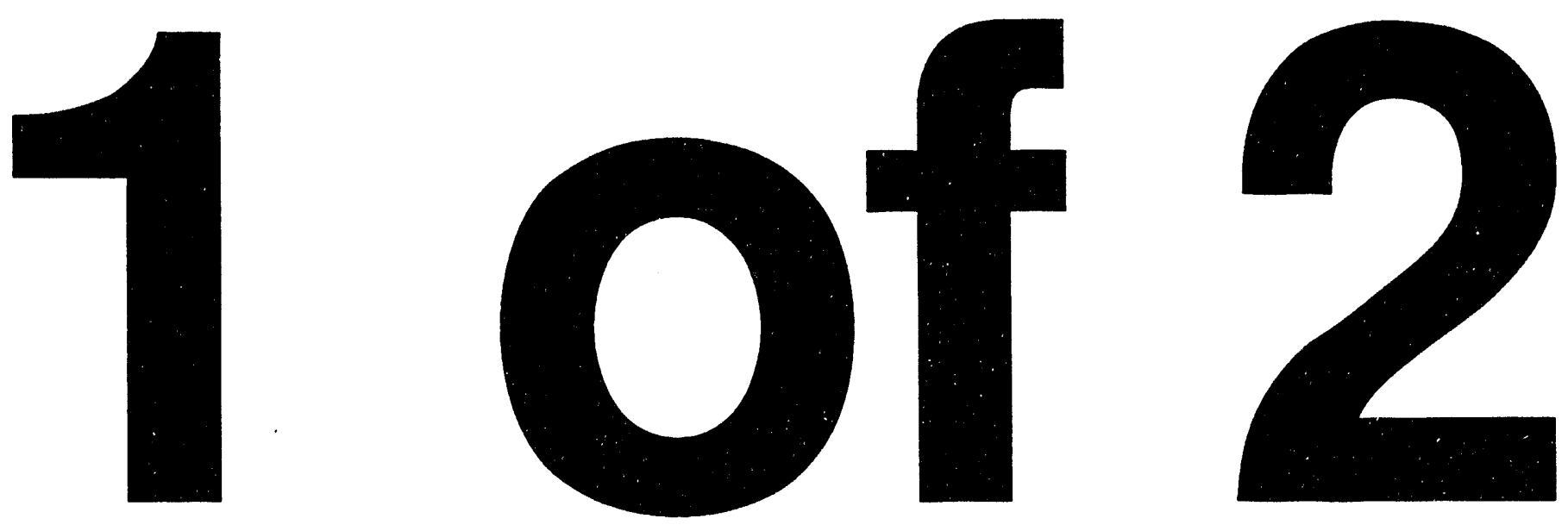


\section{An Empirical Investigation of Operator Performance in Cognitively Demanding Simulated Emergencies}

Manuscript Completed: April 1994

Date Published: July 1994

Prepared by

E. M. Roth, R. J. Mumaw, W STC

P. M. Lewis, USNRC

Westinghouse Science and Technology Center 1310 Beulah Road

Pittsburgh, PA 15235

Prepared for

Division of Systems Research

Office of Nuclear Regulatory Research

U.S. Nuclear Regulatory Commission

Washington, DC 20555-0001

NRC FIN L1505 


\begin{abstract}
This report documents the results of an empirical study of nuclear power plant operator performance in cognitively demanding simulated emergencies. During emergencies operators follow highly prescriptive written procedures. The objectives of the study were to understand and document what role higher-level cognitive activities such as diagnosis, or more generally 'situation assessment,' play in guiding operator performance, given that operators utilize procedures in responding to the events. The study examined crew performance in variants of two simulated emergencies: (1) an Interfacing System Loss of Coolant Accident and (2) a Loss of Heat Sink scenario. Data on operator performance were collected using training simulators at two plant sites. Up to 11 crews from each plant participated in each of two simulated emergencies for a total of 38 cases analyzed. Crew performance was videotaped and partial transcripts were produced and analyzed. The results revealed a number of instances where higher-level cognitive activities such as situation assessment and response planning enabled operators to handle aspects of the situation that were not fully addressed by the procedures. This report documents these cases and discusses their implications for the development and evaluation of training and control room aids, as well as for human reliability analyses.
\end{abstract}




\section{Contents}

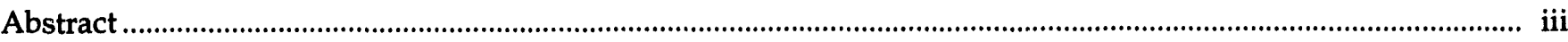

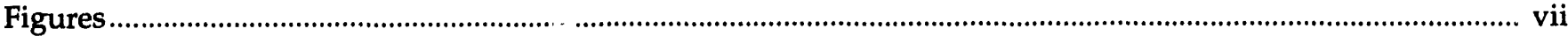

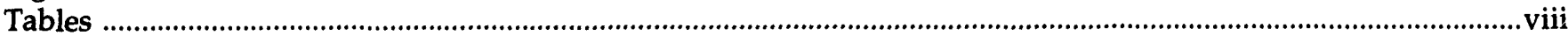

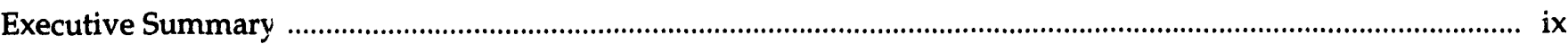

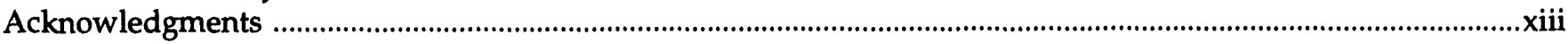

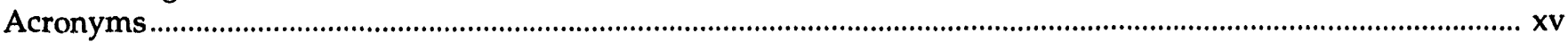

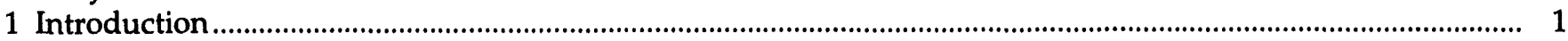

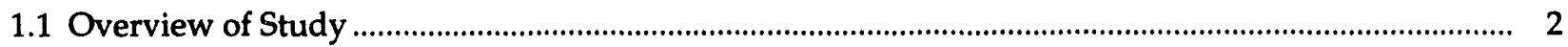

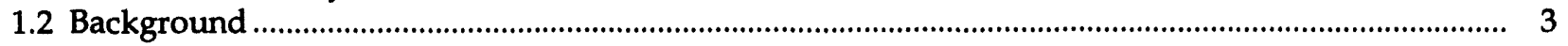

1.3 Examining Crew Interaction Skills in Cognitively Demanding Scenarios ........................................... 5

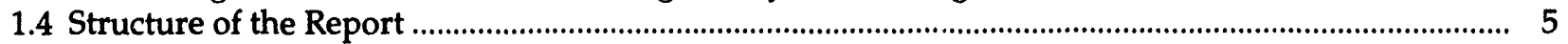

2 Study Methodology

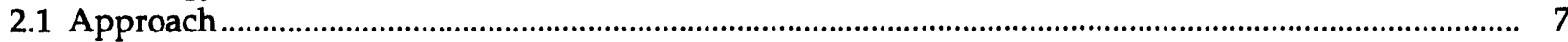

2.2 A Model of Cognitive Activities Involved in Operator Performance in Emergencies .......................... 7

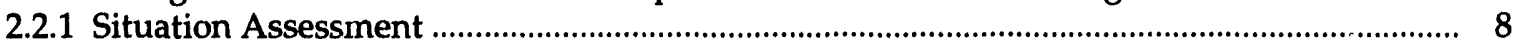

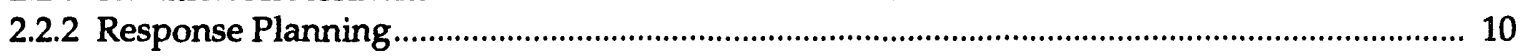

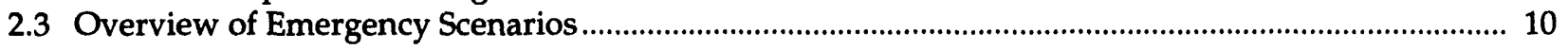

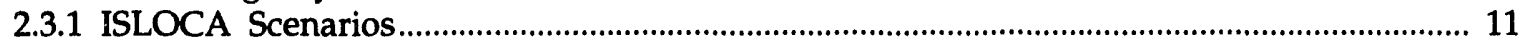

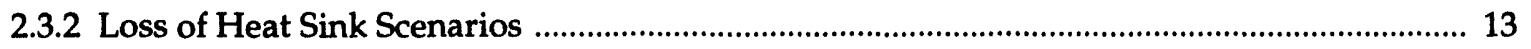

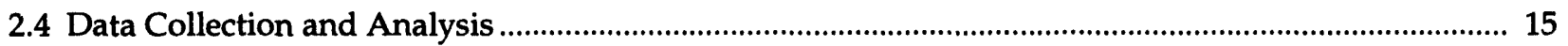

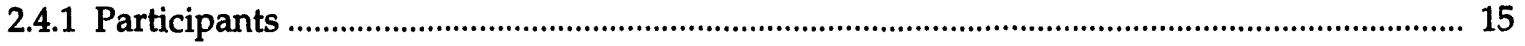

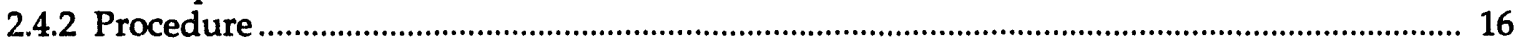

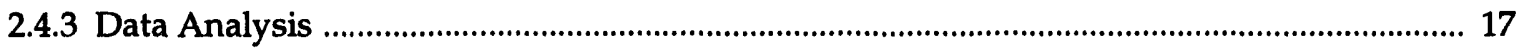

2.4.3.1 Information Recorded in the Protocol ............................................................... 17

2.4.3.2 Behaviorally Anchored Rating Scales (BARS) of Team Interaction Skills ............. 19

3 Cognitive Performance in the Simulated Emergencies ............................................................................................ 21

3.1 ISLOCA 1: ISLOCA into RHR Leading to Pipe Rupture in Auxiliary Building ................................... 21

3.1.1 Summary of Simulated Scenario ............................................................................................. 21

3.1.2 Characteristics of Participating Crews .......................................................................................... 21

3.1.3 A Case Where a Step in the EOP Explicitly Requests the Crews to Identify and Isolate a Leak ........................................................................................................................................ 22

3.1.4 A Case Where Operators Needed to Determine Whether Plant Behavior was the Result of Known Manual Actions or a Plant Fault ............................................................... 28

3.1.5 Illustrative Protocol of Crew Performance in ISLOCA 1 ........................................................... 30

3.1.6 Variability in Crew Performance .................................................................................................... 34

3.2 ISLOCA 2: ISLOCA Into RHR Leading to a Break in the RHR Heat Exchanger to the CCW .............. 35

3.2.1 Characteristics of Participating Crews ..................................................................................... 35

3.2.2 A Case Where the Procedure Containing Relevant Guidance Could not be Reached Within the EOP Transition Network ............................................................................................... 35

3.2.3 A Case Where Operators Needed to Determine Whether Plant Behavior was the Result of Known Manual Actions or a Plant Fault ................................................................ 41

3.2.4 Cases Where Operators Evaluated and Redirected the Procedure Path ................................ 41

3.2.5 Variability in Crew Performance .............................................................................................. 45

3.3 Loss of Heat Sink 1: Total Loss of Secondary Heat Sink (Feedwater Never Recovered) ...................... 46

3.3.1 Characteristics of Participating Crews ............................................................................................. 46

3.3.2 A Case Where Operators Needed to Determine Whether Plant Behavior was the

Result of Known Manual and/or Automatic Actions or the Result of a Plant Fault ............ 46 
Contents

3.3.3 A Case Where Operators Had to Decide Whether to Manually Initiate a Safety System Based on Consideration and Balancing of Multiple Goals

3.3.4 Cases Where Evaluating the Procedure Path Enabled Operators to Catch Their Own Errors

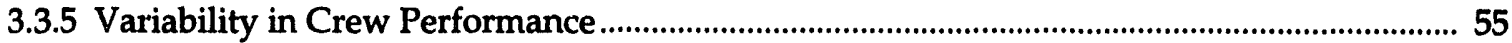

3.4 Loss of Heat Sink 2: Total Loss of Secondary Heat Sink (Feedwater Recovered) ................................ 56

3.4.1 Characteristics of Participating Crews ............................................................................................... 57

3.4.2 A Case Where Operators Needed to Determine Whether Plant Behavior was the

Result of Known Manual and/or Automatic Actions or the Result of a Plant Fault ............. 57

3.4.3 A Case Where Operators Were Kequired to Evaluate the Appropriateness of Procedure Steps Given the Specifics of the Situation ..............................................................6 60

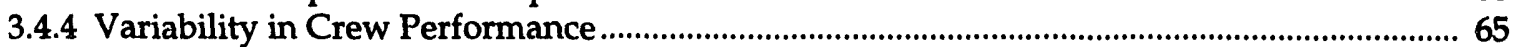

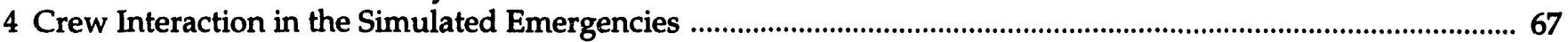

4.1 Cognitively Demanding Situations Where Good Crew Interaction was Important ............................... 67

4.1.1 Cases Where Crews Needed to Pursue Multiple Objectives........................................................ 67

4.1.2 Cases Where Situation Assessment Required Integration of Information Across Multiple Crew Members. 68

4.1.3 Cases Where Crews had to Evaluate Whether to Take Actions Outside the Procedures

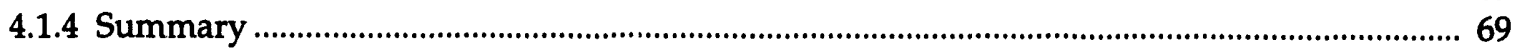

4.2 BARS Ratings of Crew Interaction Skills .................................................................................................... 70

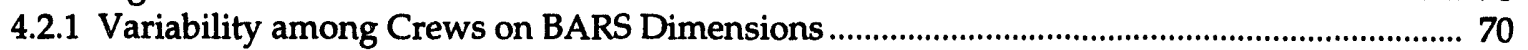

4.2.2 Evidence of a Link between Crew Interaction Skills and Technical Performance ................. 71

4.3 General Discussion of Team Interaction Skills Results ........................................................................ 72

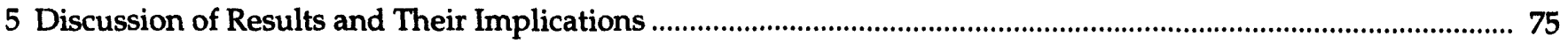

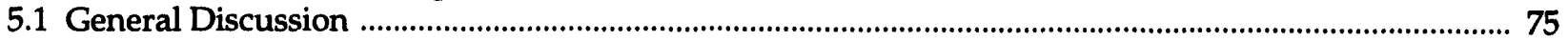

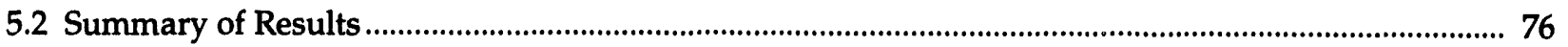

5.3 The Role of Situation Assessment and Response Planning in Cognitively Demanding

Emergencies

5.3.1 Situation Assessment

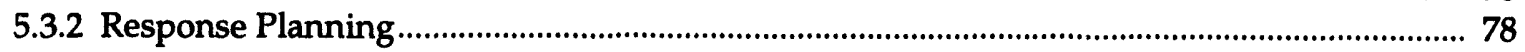

5.4 Alternative Views of the Role of Procedures and Implications of Results ............................................ 79

5.4.1 View 1: Procedures Should Provide Detailed Guidance for Every Contingency .................. 79

5.4.2 View 2: Procedures Are Not Intended to be Optimal.................................................................. 80

5.4.3 View 3: Situation Assessment and Response Planning Enable Operators to

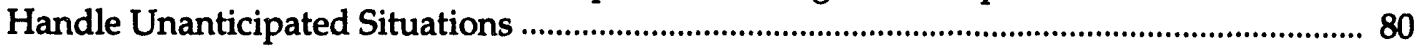

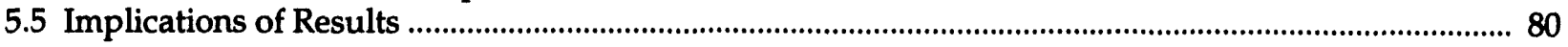

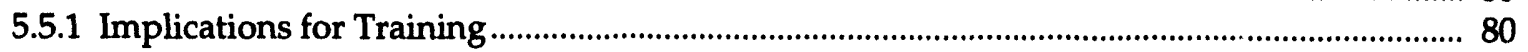

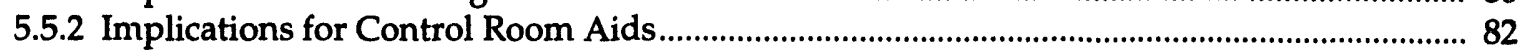

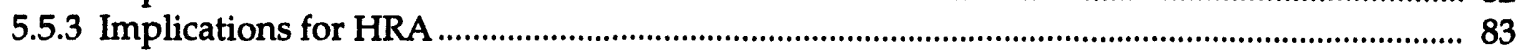

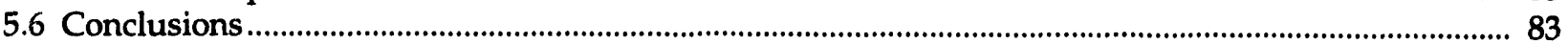

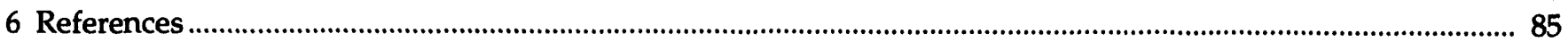

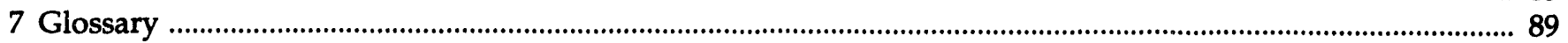

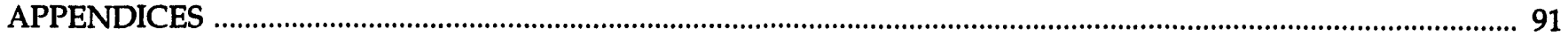

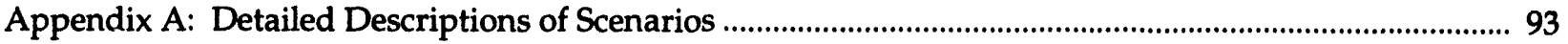

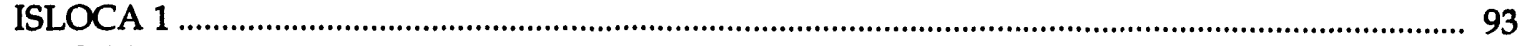

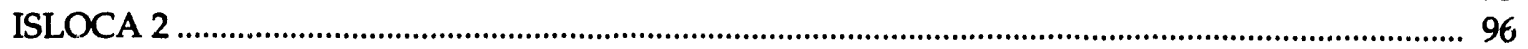

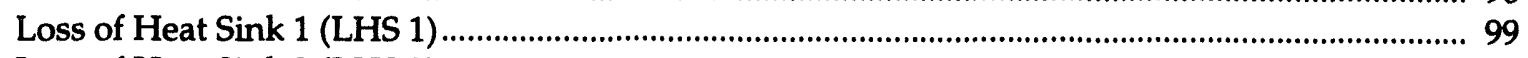

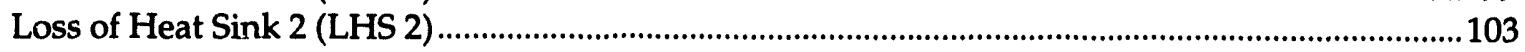

Appendix B: Instructions to Study Participants and Sample Summary Sheets...............................................107

Appendix C: Behaviorally Anchored Rating Scales (BARS) ...........................................................................113

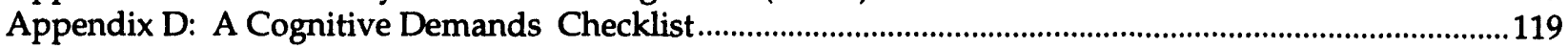




\section{Figures}

Figure 2.1 The cognitive activities encompassed under situation assessment and response planning.

Figure 2.2 Types of observable behaviors that result from situation assessment and response planning.

Figure 2.3 Simplified diagram of the systems relevant to the ISLOCA scenarios.

Figure 2.4 Simplified diagram of the systems relevant to the Loss of Heat Sink scenarios.

Figure 2.5 Illustration of the logic employed to infer situation assessment and response planning ....................... 18

Figure 3.1 EOP transitions between LOCA procedure (E-1) and SI Termination Procedure. ................................. 29

Figure 5.1 Operator knowledge required to support situation assessment and response planning. .................... 81

Figure A.1 More detailed diagram of the Residual Heat Removal System. ............................................................. 94

Figure A.2 EOP transitions relevant to ISLOCA event at Plant 1 ................................................................................ 95

Figure A.3 EOP transitions relevant to ISLOCA event at Plant 2 ...................................................................... 97

Figure A.4 Structure of Off Normal Procedure (OFN) for CCW System Malfunction............................................100

Figure A.5 EOP transitions for Loss of Heat Sink event. .....................................................................................105 


\section{Tables}

Table 1.1 A listing of crew behaviors that provided evidence of situation assessment and response planning... 3

Table 3.1 ISLOCA 1. Hypothesized explanations for plant symptoms. ............................................................... 25

Table 3.2 ISLOCA 1. Consideration of RHR train isolation. ................................................................................... 27

Table 3.3 ISLOCA 2. Crew recognition that event was not a simple LOCA........................................................ 37

Table 3.4 ISLOCA 2. Hypothesized explanation for RHR problem. .................................................................... 38

Table 3.5 ISLOCA 2. First hypothesis generated to explain the CCW problem................................................. 39

Table 3.6 LHS 1. Crew identification of a steam space leak. ..................................................................................4 48

Table 3.7 LHS 1. Decisions to close the PORV block valve. .................................................................................. 51

Table 3.8 LHS 1. Crews that considered initiating SIor going to a bleed and feed............................................... 52

Table 3.9 LHS 1. Crew decision regarding manual initiation of SI. ................................................................. 53

Table 3.10 LHS 2. Whether crews closed the pressurizer PORV block valve ............................................................ 58

Table 3.11 LHS 2. First point at which crews considered manual SI. .......................................................................6. 60

Table 3.12 LHS 2. Crew response to Reactor Trip procedure steps …........................................................................ 62

Table 3.13 LHS 2. Foldout page SI criteria met and crew response. ....................................................................... 62

Table 4.1 Mean ratings of crews on the BARS dimensions. ................................................................................... 70

Table 4.2 Mean BARS ratings for crews that differed in technical performance...................................................... 72 


\section{Executive Summary}

An empirical study was conducted to examine operator performance in cognitively demanding simulated emergencies. During emergencies operator crews are required to follow highly prescriptive Emergency Operating Procedures (EOPs). The objective of the study was to understand and document what role higher-level cognitive activities such as situation assessment and response planning play in guiding operator performance during complex emergencies, given that operators utilize EOPs in responding to the events. ${ }^{1}$

One view is that in emergencies the operators' primary role is to rotely follow the EOPs. According to this view all that is needed for successful performance is that operators be able to read and follow the individual steps in the EOPs.

Another view is that higher-level cognitive activities such as situation assessment and response planning continue to play an important role, even when EOPs are employed. According to this view the role of situation assessment and response planning is to enable crews to identify and deal with situations that are not fully addressed by the procedures.

These alternative views have very different implications for the kinds of training, procedures, displays, and decision-aids that need to be provided to control room operators. They also have different implications for the kinds of analyses that are required to assess human reliability.

The study we conducted was designed to shed light on the role of higher-level cognitive activities in guiding operator performance in cognitively demanding emergencies.

\footnotetext{
${ }^{1}$ Situation assessment is defined as constructing an explanation to account for observed plant behavior. It is similar to 'diagnosis' but broader in scope. Diagnosis typically refers to the process of searching for the cause(s) for abnormal plant behavior. Situation assessment encompasses explanations that are generated to account for plant behavior during all plant conditions (i.e., normal as weli as abnormal plant states). Response planning refers to deciding on a course of action given a particular situation assessment. These concepts are described more fully in Section 2.2 .
}

\section{Overview of Methodology}

The study examined crew performance in variants of two cognitively demanding simulated emergencies: (1) an Interfacing System Loss of Coolant Accident (ISLOCA) and (2) a Loss of Heat Sink (LHS) scenario complicated by a leaking pressurizer power operated relief valve (PORV).

Data on operator performance were collected using training simulators at two plant sites. Two utilities were asked if they would voluntarily participate in an empirical study of operator performance in cognitively complex simulated emergencies. Both agreed to run an ISLOCA and a Loss of Heat Sink event as part of the regularly scheduled requalification training exercises at one of their nuclear power plant sites. Up to 11 crews from each plant, including both actual operator crews currently on shift and staff crews, participated in each of two simulated emergencies for a total of 38 cases analyzed.

Crew performance was videotaped and partial transcripts of the crew performance were produced. These transcripts were then analyzed to:

- Identify situations where higher-level cognitive activities enabled operators to deal with aspects of the situation that were not fully handled by the procedure;

- Document behaviors the operators engaged in to handle these situations.

\section{Overview of Results}

The results of the study supported the view that crew situation assessment and response planning continue to play an important role, even when EOPs are employed. We found a number of situations where situation assessment and response planning enabled the crews to handle aspects of the situation that were 
not fully covered by the procedures. These included:

- An EOP step that explicitly requested that crews identify and isolate a leak on their own;

- A case where the procedure containing relevant guidance could not be reached within the EOP transition network;

- Cases where operators needed to determine whether plant behavior was the result of known manual and/or automatic actions (e.g., a controlled cooldown) or the result of a plant fault;

- A case where operators were required to evaluate the appropriateness of procedure steps given the specifics of the situation;

- Cases where operators had to evaluate the procedure path and take action to redirect the procedure path;

- A case where operators had to decide whether to manually initiate a safety system based on consideration and balancing of multiple goals related to safety.

In each of the simulated scenarios situations arose where operators needed to engage in situation assessment and response planning in order to handle aspects of the situation that were not fully covered by the EOPs.

In one variant of the ISLOCA scenario (ISLOCA 1) the crews were required to identify and isolate a leak into the Residual Heat Removal System (RHR) without explicit procedural guidance. In the second variant of the scenario (ISLOCA 2), while there was a procedure transition available to an ISLOCA procedure, it could not always be reached. Even in the cases where the ISLOCA rrocedure was reached, the procedure did not cover all aspects of the situation, i.e., a leak from the RHR into the Component Cooling Water System (CCW) in ISLOCA 2.

Most crews actively sought information to help identify the sources of leaks into the RHR and CCW, and identified and took actions in an attempt to isolate the leaks. They actively utilized resources beyond the EOPs, such as schematics and alarm printouts, to support their identification and isolation of the leaks. Without active situation assessment, and response planning, they would not have been able to identify and isolate the leaks.

At the same time most of the crews recognized the importance of continuing to proceed through the EOPs. They perceived getting to the Cooldown and Depressurization procedure as a high priority activity. Balancing the dual requirements to pursue the leak into the RHR with the need to proceed expeditiously through the EOPs provided one of the most challenging aspects of the ISLOCA scenarios.

The ISLOCA scenarios also provided evidence of crews actively engaging in reasoning about the procedure logic. Clear instances were found of crews reasoning at two levels. The crews were engaging in situation assessment and goal identification. At the same time they were reasoning about the strategies underlying the EOPs, and the EOP transition network logic in order to assess whether the procedure they were following would enable them to achieve plant goals in a timely manner.

We found instances where monitoring the appropriateness of the procedure path enabled crews to identify when they were in an unproductive loop, and to identify another procedure path that would allow them to take necessary actions more expeditiously.

The Loss of Heat Sink scenarios provided further evidence that complex multiple fault conditions can arise where operators need to actively engage in situation assessment and response planning. In the Loss of Heat Sink scenarios the procedures provided no guidance in identifying and responding to the leaking pressurizer PORV. The majority of crews were successfully able to detect the symptoms on the primary system and integrate them to identify the leak. This was a difficult cognitive task that required recognizing that the primary side behavior could not be entirely accounted for by the ongoing cooldown caused by efforts to recover the heat sink.

In one variant of the Loss of Heat Sink scenario (LHS 1), the crews were faced with a decision regarding manual initiation of a safety system. The only EOP guidance available to them was in a caution that indicated that they had discretion to turn on the safety system if conditions in the plant "degraded." The decision of whether to turn on the safety system 
required balancing multiple goals. Manual initiation of the safety system would respond effectively to the degrading conditions in the primary system caused by the leaking PORV, but could potentially delay recovery of heat sink. The crews had some difficulty with this aspect of the scenario. Most of the crews did not recognize that they had the discretion to decide whether to turn on the safety system. Further, few of the crews showed evidence of considering the tradeoffs involved. The majority of crews chose to let conditions continue to degrade until a criterion was reached for which more explicit procedural guidance was available.

The second variant of the Loss of Heat Sink scenario (LHS 2) provided additional opportunity to examine the role of situation assessment in guiding crew performance. In this scenario a case arose where operators had to decide the appropriateness of specific procedure steps based on their own situation assessment. In LHS 2 the crews recovered feedwater on the secondary side, thus restoring the heat sink. As required by the EOPs they then returned to the procedure that had been in effect prior to the loss of heat sink, which was the reactor trip procedure. This procedure contained some steps that required them to undo actions they had just taken to recover feedwater. If they followed those steps it would result in a loss of heat sink again. The EOP background document explicitly recognized that this type of situation could arise and indicated that in those cases operator judgment would be required in determining appropriate action.

Most of the crews correctly recognized that some of the steps in the reactor trip procedure were inappropriate to the situation and should not be followed. This included steps that called for ini'iation of a safety system. The decision that initiation of the safety system was not needed was based on situation assessment. The crews had to determine whi ther the conditions in the primary system were due to cooldown or a plant fault. This was not a simple determination, as attested by the fact that, in the case of two of the crews who faced that decision, there was a leak present (leaking pressurizer PORV), but the crews nevertheless initially attributed the primary side symptoms to cooldown, and decided against manual initiation of the safety system.

While most of the crews performed well, variability in performance was observed in all the scenarios.
Crews differed in the extent to which they detected plant symptoms, actively sought an explanation for unexpected findings, and attempted to come up with a coherent explanation that accounted for all the observed symptoms. In each scenario there was at least one crew that had difficulty identifying the source of the problem and taking appropriate action to mitigate it (i.e., approximately $10 \%$ of crews run in the event). The fact that not all crews in the scenarios formed the correct situation assessment suggests that there is room for improvement.

The results also clarified the role of group interaction in situation assessment and response evaluation, and provided suggestive evidence of the conditions under which crew interaction skills may be expected to affect technical performance of crews.

\section{Overview of Conclusions}

The results of this study provide support for the position that situation assessment and response planning continue to be important for successful operator performance, even when EOPs are employed.

In our scenarios a number of cognitively demanding situations arose where situation assessment enabled operators to handle aspects of the situation that were not covered by the procediures. While these cases were drawn from variants of two specific emergency scenarios, we believe that the types of situations we identified are generic classes that are likely to arise in other emergency scenarios. It is reasonable to assume that similar situations may arise in actual events. In such situations the ability of operators to form accurate situation assessments and to generate response plans to cover aspects of the situation that are not fully addressed by the procedures will be important.

The results of the study, taken in combination with evidence from actual incidents, and experiences in related domains support the position that situation assessment and response planning enable operators to handle unanticipated situations that are not fully addressed by procedures.

The conclusion that situations may arise where crews need to engage in situation assessment and response 
planning has implications for the development and evaluation of training and control room aids, as well as for human reliability analyses (HRA). With respect to training, the results suggest that explicit attention may need to be paid to the development of these cognitive skills. The results also have potential implications for the development and evaluation of control room aids. In particular, they suggest potential value for displays and decision-aids that are explicitly intended to support situation assessment and response planning.

The results also have potential implications for HRA. They suggest that human reliability assessments are likely to be more accurate if the dynamics of the event, and the factors that are likely to complicate situation assessment and response planning, are explicitly considered in performing the analyses. A Cognitive Demands Checklist that can be used to support HRA is included in Appendix D.

A final conclusion of the study regards the value of empirical studies of operator performance in simulated emergencies for addressing human performance issues of concern to the NRC. Well designed empirical studies can provide specific, clear conclusions for practical decision making. The present study illustrates how empirical studies of operator performance in simulated emergencies can be used to investigate a human performance issue -in this case the role of higher-level cognitive activity in operator response to cognitively demanding emergencies. The study provided (1) evidence that situations can arise where higher-level cognitive activity on the part of operators is needed and (2) objective data on how different operator crews responded to these situations. 


\section{Acknowledgments}

We wish to gratefully acknowledge the enthusiastic support provided by the management and staff at the two utilities that participated in the simulator study. Without their voluntary participation and support, this type of study would not have been possible. The utilities generously provided access to their training simulator and operating crews, and allowed us to run and videotape specially tailored scenarios as part of their requalification training.

Everyone at the two utilities was exceedingly supportive and worked hard to accommodate the needs of the study. We particularly wish to acknowledge the training simulator personnel who modified the simulator models to accommodate the requirements of our scenarios.

We wish to especially thank the training instructors at the two utilities who contributed substantively to the design of the simulator scenarios and were exceedingly conscientious in ensuring that the scenarios were run in a controlled manner, and that the videotapes, data tapes, and summary data sheets were properly recorded. In a very real sense they deserve credit as co-investigators in this study.

We also wish to acknowledge the contributions of a number of people at Westinghouse. We wish to thank Robert G. Orendi, of the Plant Operations and Evaluations department at the Westinghouse Nuclear Technology Division (NTD), for his role in providing experi consultation on Nuclear Power Plant operations and emergency operating procedures. We also wish to triank Robert. J. Lutz and Debra Ohkawa of the Engineering Technology Department at NTD for generously giving of their time and their patience in answering questions about plant behavior and operating practice. We would also like to acknowledge Roger A. Mundy, of the Nuclear and Technology Department at NTD for processing the plant parameter data tapes recorded from the high fidelity training simulators. We are also grateful to Donna L. Rutt, of the Human Sciences group at the Westinghouse Science \& Technology Center (STC), for her support in transcribing the videotapes of crew performance during the simulated emergencies and in preparation of the final report. Finally, we are most grateful to Doris A. Pollitt, of the Business Operations Department at STC, who served as technical editor, for her patience and excellent advice.

We also wish to acknowledge the role of Dr. Harry E. Pople, Jr. of Seer Systems. Dr. Pople actively contributed to the design of the empirical studies and participated in the plant data collection visits. His insights regarding the factors that influence operator cognitive performance and ideas about cognitive modeling were extremely stimulating and influenced many of the ideas presented here.

Finally, we wish to thank the operator and staff crews that participated as subjects in the study for allowing us to observe and report on their performance. 


\section{Acronyms}

$\begin{array}{ll}\text { AI } & \text { Artificial Intelligence } \\ \text { BARS } & \text { Behaviorally Anchored Rating Scale } \\ \text { BIT } & \text { Boron Injection Tank } \\ \text { BOP } & \text { Balance of Plant Operator } \\ \text { CT } & \text { Cannot Tell } \\ \text { CES } & \text { Cognitive Environment Simulation } \\ \text { CCP } & \text { Centrifugal Charging Pump } \\ \text { CCW } & \text { Component Cooling Water } \\ \text { CREATE } & \text { Cognitive Reliability Assessment Technique } \\ \text { EDO } & \text { Emergency Duty Officer } \\ \text { EOP } & \text { Emergency Operating Procedures } \\ \text { ERG } & \text { Emergency Response Guidelines } \\ \text { FRG } & \text { Functional Restoration Guidelines } \\ \text { HRA } & \text { Human Reliability Analyses } \\ \text { ISLOCA } & \text { Interfacing System Loss of Coolant Accident } \\ \text { LHS } & \text { Loss of Heat Sink } \\ \text { LOCA } & \text { Loss of Coolant Accident } \\ \text { N/A } & \text { not applicable } \\ \text { LUPD } & \text { Level Trending Up with Pressure Trending Down } \\ \text { NATD } & \text { Nuclear and Advanced Technology Division } \\ \text { NPP } & \text { Nuclear Power Plant } \\ \text { NRC } & \text { U. S. Nuclear Regulatory Commission } \\ \text { OFN } & \text { Off Normal Procedure } \\ \text { PORV } & \text { Power Operated Relief Valve } \\ \text { PRA } & \text { Probabilistic Risk Assessment } \\ \text { PRT } & \text { Pressurizer Relief Tank } \\ \text { PRZR } & \text { Pressurizer } \\ \text { PWR } & \text { Pressurized Water Reactor } \\ \text { RCS } & \text { Reactor Coolant System } \\ \text { RHR } & \text { Residual Heat Removal } \\ \text { RNO } & \text { Response Not Obtained } \\ \text { RO } & \text { Reactor Operator } \\ \text { RVLIS } & \text { Reactor Vessel Level Indication System } \\ \text { RWST } & \text { Refueling Water Storage Tank } \\ \text { SG } & \text { Steam Generator } \\ \text { SI } & \text { Safety Injection } \\ \text { SO } & \text { Supervising Operator } \\ \text { SRO } & \text { Senior Reactor Operator } \\ \text { SS } & \text { Shift Supervisor } \\ \text { STA } & \text { Shift Technical Advisor } \\ \text { STC } & \text { Science \& Technology Center } \\ & \end{array}$




\section{Introduction}

Human performance is a significant contributor to nuclear power plant (NPP) safety (e.g., Trager, 1985; Kauffman, Lanik, Trager, and Spence, 1992). During emergency situations operator action can have a substantial impact on the ability to return the plant to safe operation. Operators may take recovery actions that mitigate the emergency situation. Alternatively, errors in performance can delay or hinder plant recovery.

Examination of actual incidents both inside and outside the NPP industry indicates that incidents often involve complicating factors (e.g., failed sensors; multiple faults) that impose difficult cognitive demands on operators (Perrow, 1984; Wagenaar and Groeneweg, 1987; Reason, 1990; Woods, Johanriesen, Cook, and Sarter, 1993). Complications include sensor failures that make situation assessment difficult, cases where available procedures do not map well to the specifics of the situation, and situations where balancing of multiple goals related to safety is required (e.g., NRC, NUREG-1154; NRC, NUREG-1455; Kauffman et al., 1992).

As part of a U.S. Nuclear Regulatory Commission (NRC) project to model the cognitive activities that underlie NPP operator performance in emergencies, an empirical study was conducted to examine operator performance in cognitively demanding simulated emergencies. This report presents the results of the empirical study.

During emergencies operator crews are required to follow highly prescriptive Emergency Operating Procedures (EOPs). The objective of the study was to understand and document what role higher-level cognitive activities such as situation assessment and response planning play in guiding operator performance during complex emergencies, given that operators utilize EOPs in responding to the events.

In an emergency the role of the operator is to ensure plant safety. The operator monitors automatic plant safeguard systems, initiates recovery actions to minimize radiation release and equipment damage and return the plant to a stable condition, and ensures that critical safety functions are maintained.
EOPs provide predefined strategies for accomplishing these functions. When an emergency arises that causes the reactor to trip, the operators are required to take out the EOPs and follow the procedures step by step. The EOPs provide detailed guidance on what plant parameters to check, how to interpret the symptoms observed, and what control actions to take.

Given that operators utilize highly prescriptive procedures in responding to emergencies, a question arises regarding the nature and extent of cognitive activity required of operators to adequately handle emergencies.

One view is that all that is needed of operators is that they understand and follow the steps in the EOP. Under this view what is needed for successful performance is that operators be able to read and understand the individual steps in the procedure, that they be able to locate and read the plant parameter values specified in the procedure steps, and that they be able to locate the controls and take the actions indicated in the procedure steps.

Another view is that higher-level cognitive activities such as situation assessment and response planning continue to be important for successful operator performance, even when EOPs are employed. Under this view the role of situation assessment and response planning is to enable crews to identify and deal with situations that are not fully addressed by the procedures.

These alternative views have very different implications for the kinds of training, procedures, displays and decision-aids that need to be provided to operators. They also have very different implications for the kinds of analyses that are required to assess human reliability.

The study we conducted was designed to shed light on the role of higher-level cognitive activities in guiding operator performance in emergencies. The design and analysis of the study was guided by a model of higher-level cognitive activities involved in operator performance in emergencies. The model described how situation assessment and response 
planning was expected to affect operator performance in emergencies. The model helped to specify cognitively demanding scenarios where the influence of situation assessment and response planning on operator behavior would be most readily observed, and specified the kinds of operator behaviors to look for as evidence of these higher-level cognitive activities.

\subsection{Overview of Study}

The study examined crew performance in two cognitively demanding simulated emergencies: (1) an Interfacing System Loss of Coolant Accident (ISLOCA) and (2) a Loss of Heat Sink (LHS) scenario complicated by a leaking pressurizer power operated relief valve (PORV).

Data on operator performance were collected using training simulators at two plant sites. Two utilities were asked if they would voluntarily participate in an empirical study of operator performance in cognitively complex simulated emergencies. Both agreed to run an ISLOCA and a Loss of Heat Sink event as part of the regularly scheduled requalification training exercises at one of their nuclear power plant sites. In this report the plants are referred to as Plant 1 and Plant 2. A different variant of the events was run at each plant.

Up to 11 crews from each plant, including both actual operator crews currently on shift and staff crews, participated in each of two simulated emergencies for a total of 38 cases analyzed.

Crew performance was videotaped and partial transcripts of the crew performance were produced. These transcripts were then analyzed to:

- Identify situations that arose where operators needed to engage in higher-level cognitive activities in order to deal with the situation;

- Document behaviors the operators engaged in to handle those situations that were not explicitly directed by a specific EOP step (henceforth referred to as extra-procedural activities).

The extra-procedural activities provided evidence of situation assessment and response planning.
The analysis identified six kinds of situations where operators needed to engage in situation assessment and response planning in order to adequately deal with the situation. These situations are listed below with the simulated scenarios in which they arose presented in parentheses.

- An EOP step that explicitly requested that crews identify and isolate a leak (ISLOCA 1);

- A case where the procedure containing relevant guidance could not be reached within the EOP transition network (ISLOCA 2);

- Cases where operators needed to determine whether plant behavior was the result of known manual and/or automatic actions (e.g., a controlled cooldown) or the result of a plant fault (all four simulated events);

- A case where operators were required to evaluate the appropriateness of procedure steps given the specifics of the situation (LHS 2);

- Cases where operators had to evaluate the appropriateness of a procedure path and take action to redirect the procedure path (ISLOCA 2; LHS 1; LHS 2);

- A case where operators had to decide whether to manually initiate a safety system based on consideration and balancing of multiple goals related to safety (LHS 1).

Examination of crew performance in these situations revealed clear instances of crews:

- Actively engaging in situation assessment and goal identification;

- Actively monitoring the appropriateness of steps in the EOPs for achieving progress toward these identified goals; and,

- Adapting the procedures to the situation.

Table 1.1 presents the types of extra-procedural behaviors that were observed in the study and the scenarios in which the clearest examples of each type 
Table 1.1 A listing of crew behaviors that provided evidence of situation assessment and response planning, and the scenarios in which clear examples of those behaviors were observed.

\section{Situation Assessment:}

Checking for evidence to confirm hypothesis

Explaining observed symptoms

Identifying unexpected plant behaviors

Detecting abnormal plant behaviors

Identifying problems (e.g., plant malfunctions)

Detecting alarms/symptoms that had been missed

\begin{tabular}{|c|c|c|c|}
\hline- & - & - & $\sqrt{ }$ \\
\hline$\sqrt{ }$ & $\sqrt{ }$ & $\sqrt{ }$ & - \\
\hline- & $\sqrt{ }$ & - & $\sqrt{ }$ \\
\hline$\sqrt{ }$ & - & $\sqrt{ }$ & $\sqrt{ }$ \\
\hline$\sqrt{ }$ & $\sqrt{ }$ & $\sqrt{ }$ & $\sqrt{ }$ \\
\hline$\sqrt{ }$ & - & - & - \\
\hline
\end{tabular}

Response Planning:

Identifying goals

Evaluating appropriateness of EOP procedure path

Evaluating consequences of actions

Adapting procedures to the situation

Catching errors

of behavior were found. These specific cases demonstrated the role that situation assessment and response planning played in enabling the crews to handle these cognitively demanding scenarios.

While the evidence for the role of higher-level cognitive activities in directing operator performance is drawn from particular situations that arose in the two specific emergency scenarios that we ran, the types of situations we identified represent generic classes that are likely to arise in other emergency scenarios that involve different EOP procedures. The conclusions regarding the role that higher-level cognitive activities play in guiding operator performance in emergencies can reasonably be generalized beyond the particular scenarios and crews that we observed.

A main conclusion of the study is that, while EOPs have greatly reduced the need for operators to develop diagnostic and response strategies on their own in real time, they have not eliminated the need for operators to form their own situation assessment and evaluate the effectiveness of the response strategies embodied in the procedures in dealing with the specifics of the situation. These conclusions have potential implications for advanced man-machine interfaces, computerized procedures, operator training, and human reliability analyses (HRA).

\subsection{Background}

The empirical study reported here was part of a larger project that was initiated by the U.S. NRC to study and model the cognitive activities that underlie operator performance during NPP emergencies. The project included two inter-related activities: (1) analyses of human crew performance during simulated emergencies and (2) development of an artificial intelligence (AI) computer simulation, called Cognitive Environment Simulation (CES), that simulates some of the cognitive activities involved in responding to a NPP emergency situation (NUREG/CR-4862; NUREG/CR-5213; Roth, Woods, and Pople, 1992). 
As part of the CES development process, small-scale studies of human performance in simulated emergencies were conducted using crews composed of training instructors. Results of these small-scale studies suggested that higher-level cognitive activities play a substantial role in guiding operator performance in complex emergencies, even when EOPs are used. A primary motivation for conducting the larger-scale study of operator performance in simulated emergencies described in this report was to establish the generality of these preliminary results and provide a clearer characterization of the role that cognitive activities play in guiding operator response in cognitively demanding eriergencies.

In one case CES was exercised on two variants of an ISLOCA into the residual heat removal system (Roth, Woods and Pople, 1992). Protocols recording the responses of CES to the ISLOCAs were produced and compared to the performance of human crews on the same events. Both parallels and differences in the performance of CES and the human crews were found. CES and human crews both attended to the same evidence, accessed the same types of NPP knowledge, and followed the same line of reasoning in identifying the ISLOCA. However, CES was able to detect disturbances sooner, and follow implications of disturbances more thoroughly than the human crews did.

One of the striking differences in the performance of CES and the human crews was that human crew activity was strongly governed by the EOPs whereas the diagnostic performance of CES was not restricted in the same way. While human crews displayed active situation assessment and response planning, these activities occurred primarily during periods where the demands of following the procedures were low.

The results of the ISLOCA study suggested that higher-level cognitive activities were needed to handle complex scenarios such as the ISLOCA events -- even when EOPs are used. However, the extent to which the results could be generalized was not clear because of several iimitations of the data on human performance. In particular: (1) only one type of event, an ISLOCA, was used; (2) the crews were composed of training instructors rather than actual operators; (3) only one crew was run on each event; (4) the crews were made up of two individuals rather than the standard three- to five-person crew. As a result, it was decided to perform a more extensive empirical study of operator performance in cognitively complex emergencies in order to confirm and expand the results obtained in the ISLOCA exercises. 2

The empirical study reported here was designed to address some of the limitations of the earlier smallscale ISLOCA study. The current study includes a larger set of scenarios. The two ISLOCA variants that were used in the CES exercises are replicated. In addition, two new scenarios -- two variants of a Loss of Heat Sink event - are used. The study also uses a larger, and more representative, set of operator crews as participants. Up to eleven crews were run in each event. These crews included all operator crews currently on shift and all staff crews undergoing requalification training at each of two plants. The crews were composed of four to five operators, including a shift technical advisor and a shift supervisor, which is more representative of the crew size and structure that would normally participate in an emergency event.

The fact that the current study includes a larger set of emergency scenarios, and a larger, more representative, sample of operator crews, makes it possible to generalize to other crews and types of emergency scenarios with more confidence. Because the study participants included all the operator crews currently on shift as well as staff crews undergoing retraining at each of two plants, the range of crew performance observed on the events should be reasonably representative of the range of performance of operator crews as a whole.

\footnotetext{
2 A second activity initiated under the NRC project was an attempt to enhance the capabilities of CES to model operators' use of EOPs in handling emergencies. This activity depended on upgrades to the EAGOL software (on top of which the CES model was built). While some progress was made in incorporating knowledge of procedures in EAGOL, complete CES runs demonstrating enhanced procedure-following capabilities could not be produced within the time frame of this project. Upon the recommendation of NRC senior managers, emphasis of the project was shifted from further development of CES to the empirical study.
} 


\subsection{Examining Crew Interaction Skills in Cogı.itively Demanding Scenarios}

In addition to examining the role of higher-level cognitive activity in guiding operator performance, we also examined the role of crew interaction in handling the cognitively demanding scenarios. Under a separate program sponsored by the U.S. NRC, Montgomery et al. (1992) identified six dimensions of team interaction skill, and developed Behaviorally Anchored Rating Scales (BARS) for measuring crew performance on those dimensions. In this study we examined crew performance in the scenarios to identify cognitively demanding situations that arose where good crew interaction skills appeared to be important for successful performance from a technical perspective (i.e., for correctly identifying plant malfunctions and taking appropriate action). We identified particular crew behaviors that characterized good performance on BARS dimensions, and appeared to be important for successful technical performance on the scenarios.

The analysis particularly focused on how crews organized themselves to manage the dual requirements of (1) following through the steps in the EOPs and (2) engaging in extra-procedural activities in order to handle aspects of the situation that were not covered by the EOPs. We focused on examining how different crews divided up these dual responsibilities, and whether differences in technical performance resulted.

A second aspect of the analysis focused on the usefulness of the BARS rating scales per se in evaluating team interactions skills in these scenarios. We examined crew ratings on the BARS scales to assess (1) whether there was variability in crew scores on the BARS dimensions, and (2) whether there was a relationship between BARS ratings of team skill and crew performance on the scenarios from a technical perspective.

\subsection{Structure of the Report}

This report documents the empirical study that was conducted and the conclusions drawn. Tne report is divided into five sections.

Section 1 provides an overview of the study and describes the general background and motivation for conducting the study.

Section 2 describes the methodology that was used in conducting and analyzing the study. Included is a discussion of a simplified model of higher-level cognitive activities involved in operator performance in emergencies that provided the conceptual framework for the analysis of crew performance in the simulated events.

Section 3 presents the main results on operator cognitive performance in the study. The results are organized around the six types of situations that were identified where higher-level cognitive activity was needed to handle the situation.

Section 4 presents results on team performance. It includes a discussion of how multiple crew members contributed to situation assessment and response identification and evaluation activities. It also discusses how crews organized themselves to deal with the dual needs to (1) engage in extra-procedural activities in order handle aspects of the situation that are not covered by the EOPs and (2) proceed through the EOP steps in order to ensure that all major safety functions are maintained, and that actions required to return the plant to a safe state are performed in a timely manner. Section 4 includes presentation and discussion of ratings of team interaction skills that were made using the BARS scales.

Section 5 summarizes the results of the study and discusses conclusions and implications.

Appendices $A$ through $C$ provide more details on the study methodology. Appendix A provides detailed descriptions of the scenarios. Appendix B presents the instructions and sample summary sheets that were used in conducting the study. Appendix C presents the BARS team interaction skills rating scales. 
Introduction

Appendix D presents a Cognitive Demands Checklist that provides a structured list of factors (e.g.,

characteristics of the event, the procedures, the manmachine interface) that can result in cognitive errors. The Cognitive Demands Checklist was developed to capture some of the findings of the project in a form that can be used directly by the NRC staff to assess characteristics of an accident sequence or situation that make errors of intention more likely. 


\section{Study Methodology}

\subsection{Approach}

The design and analysis of the study was guided by a model of higher-level cognitive activities involved in operator performance in NPP emergencies. The model guided the design of cognitively demanding emergency scenarios that challenged operator situation assessment and response planning. It also specified the kinds of operator behaviors to look for as evidence of situation assessment and response planning. This model is presented in Section 2.2.

Two types of simulated emergencies were included in the study: two variants of the ISLOCA into the Residual Heat Removal system (RHR) scenario that was run as part of the cognitive environment simulation (CES) exercises, and two variants of a loss of heat sink (LHS) event complicated by a stuck open pressurizer power operated relief valve (PORV). The ISLOCA scenarios were designed to be challenging from the point of view of situation assessment. The loss of heat sink scenarios were designed to be challenging both with respect to situation assessment and response planning. An overview of these scenarios is provided in Section 2.3. More detailed descriptions are provided in Appendix A.

Two utilities were asked if they would voluntarily participate in an empirical study of operator performance in cognitively complex simulated emergencies. Both agreed to run an ISLOCA and a Loss of Heat Sink event as part of the regularly scheduled requalification training exercises at one of their nuclear power plant sites. In this report the plants are referred to as Plant 1 and Plant 2. A different variant of the events was run at each plant.

The events were run on a high fidelity training simulator at the plant site. Crew performance in the simulated emergencies was videotaped. Partial transcripts tracing crew performance in each event were generated from the videotapes.
The protocols were then analyzed to:

- Identify situations that arose where operators needed to engage in higher-level cognitive activities in order to deal with the case;

- Document extra-procedural activities that operators engaged in that provided evidence of these higher-level cognitive activities.

The model of cognitive activity provided the framework for linking the specific extra-procedural activities observed to the higher-level cognitive activities.

Descriptions of the data collection and analysis methods are provided in Section 2.4 .

\subsection{A Model of Cognitive Activities Involved in Operator Performance in Emergencies}

The design and analysis of the study was guided by a model of higher-level cognitive activities involved in operator performance in NPP emergencies. The model we used draws on concepts that underlie the CES model. These concepts are consistent with the core body of knowledge and theory in cognitive psychology and are supported by a large empirical base on human decision-making in real world settings. 3

The model includes two components: situation assessment and response planning. Describing cognitive processes in terms of these two higher-level cognitive activities is consistent with standard ways of modeling decision-making (e.g., Orasanu, Dismukes and Fischer, 1993). Figure 2.1 provides a

\footnotetext{
${ }^{3}$ More detailed reviews of concepts from cognitive psychology and their application to NPP crew performance can be found in Mumaw, Swatzler, Roth and Thomas, 1994 and Woods and Roth, 1986.
} 


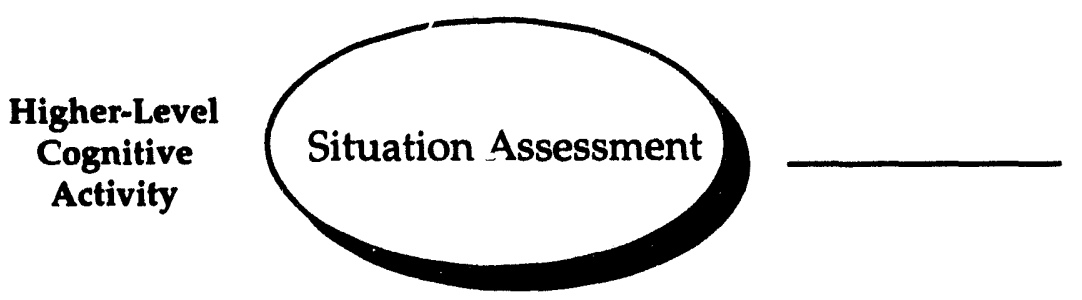

Constructing an explanation to account for observed plant behavior: a mental representation of factors known or hypothesized to be affecting plant state.

This mental representation generates:

- expectations about other plant param ters

- expectations about future consequences

- explanation for observations

- identification of unexpected plant behavior and search for explanation

- anticipation of potential future problems

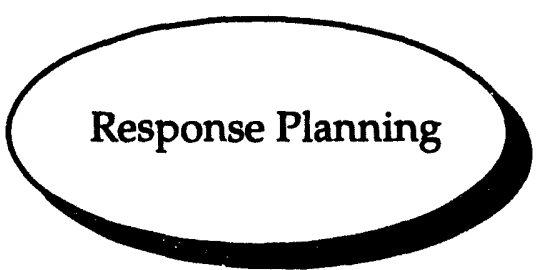

Deciding on a course of action given a particular situation assessment.

Involves:

- establishing goals

- identifying/generating a response plan

- evaluating/monitoring effectiveness of response plan

- filling in gaps in response plan

- adapting response plan

Figure 2.1 The cognitive activities encompassed under situation assessment and response planning.

summary of the cognitive activities encompassed under these two labels. Figure 2.2 shows the types of observable behaviors that result from these cognitive activities.

Below, we describe in more detail the processes involved in situation assessment and response planning and the resulting observable behaviors. Section 3 contains specific examples of cases where these types of behaviors were observed in the simulated emergency scenarios.

\subsubsection{Situation Assessment}

A fundamental finding in the field of cognitive psychology is that people actively try to construct a coherent explanation to account for their observations (Bartlett, 1932; Bransford, 1979). The process of constructing an explanation to account for observations is referred to as situation assessment. In the context of NPP operations, situation assessment involves developing and updating a mental representation of the factors known or hypothesized to be affecting plant state at a given point in time. 4 Situation assessment refers to both the process of building the mental representation and the resulting mental representation.

Situation assessment is similar in meaning to 'diagnosis' but is broader in scope. Diagnosis typically refers to searching for the cause(s) of abnormal symptoms (e.g., a disease, a malfunctioning piece of equipment). Situation assessment encompasses explanations that are generated to account for normal as well as abnormal conditions. It is similar to the concept of 'situation awareness' used in the aviation literature (Endsley, 1993; Sarter and Woods, 1991).

\footnotetext{
4 A situation assessment need not be complete or fully accurate.
} 


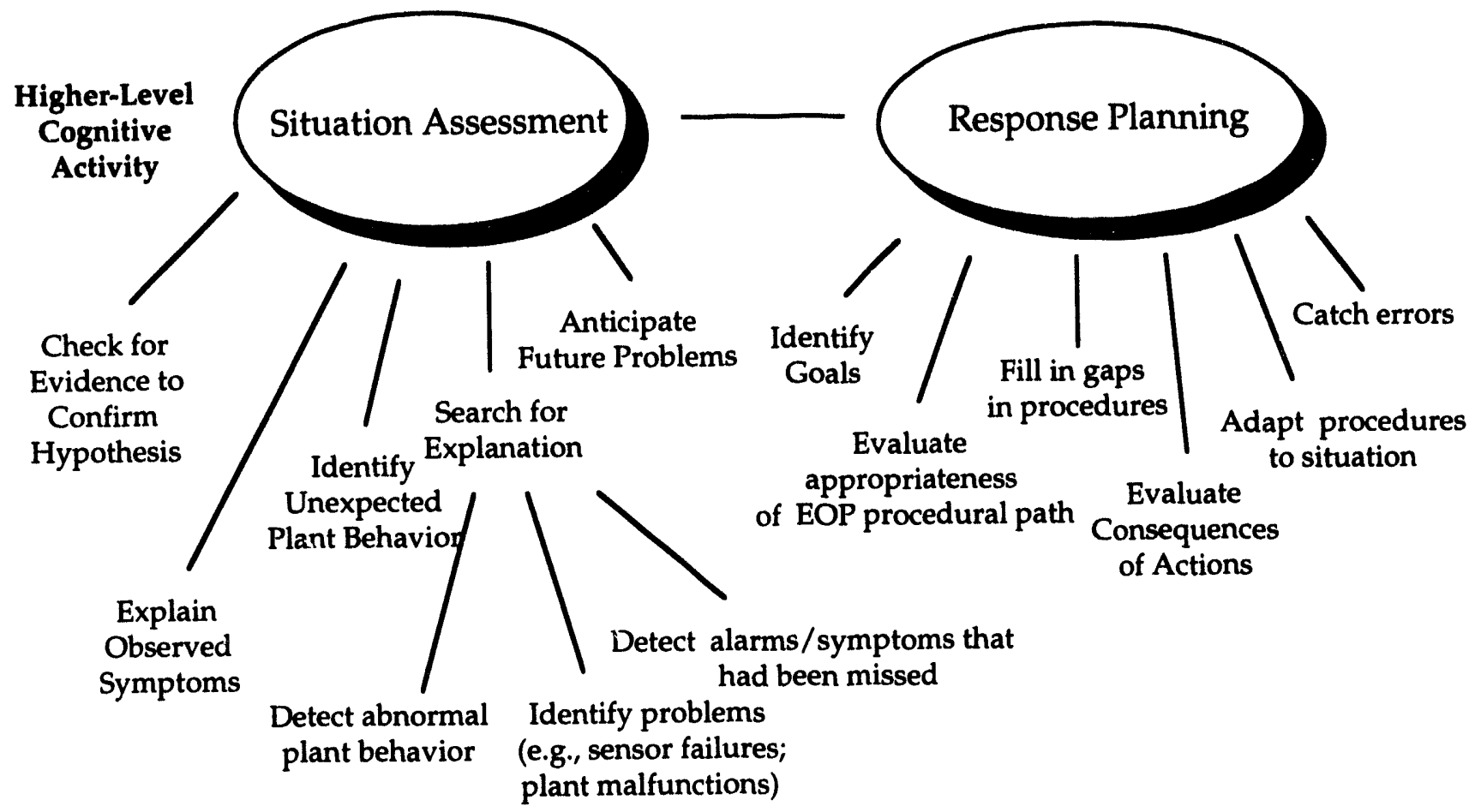

Figure 2.2 Types of observable behaviors that result from situation assessment and response planning. Evidence of these operator behaviors can be obtained through observation of operator actions and utterances

Situation assessment is a complex activity that may entail using mental models of physical systems and how they work. Examples from a nuclear power plant application include considering the physical interconnections among systems (e.g., considering piping and valve interconnections to figure out how water from one system could get into another), and considering the effects of mass and energy changes in one system on the behavior of a second system (e.g., the effect of a cooldown in the primary system on secondary side steam generator level behavior).

People form expectations based on their current situation assessment. These expectations include the events that should be happening at the same time, how events should evolve over time, and effects that may occur in the future (i.e., expectations about future consequences).

They use these expectations in several ways. They use them to search for evidence to confirm their current situation assessment. This search is a form of knowledge-driven monitoring. 5

They also use expectations they have generated to explain observed symptoms. If a new symptom is observed that is consistent with their expectations, they have a ready explanation for the finding. This gives them greater confidence in their situation assessment.

When a new symptom is inconsistent with their expectation, it is recognized as an unexpected plant behavior that suggests a need to revise the situation assessment. This symptom triggers a search for a better explanation of the situation, which may

\footnotetext{
${ }^{5}$ Knowledge-driven monitoring refers to monitoring that is driven by an internally generated perceived need for a piece of information. This is contrasted with data-driven monitoring that is triggered by salient external stimuli such as alarms, and procedure-driven monitoring that is determined by procedures that include explicit directives to monitor a parameter.
} 
involve developing a hypothesis for what might be occurring, and then searching for evidence in the environment to confirm that hypothesis (i.e., knowledge-driven monitoring). It can result in detecting abnormal plant behavior that might not otherwise have been observed, detecting plant symptoms and alarms that may have otherwise been missed, and identifying problems such as sensor failures or plant malfunctions.

People also use these expectations to project into the future and anticipate potential problems. These projections are used in generating and evaluating response plans (Klein and Calderwood, 1991).

Situation assessment allows operators to detect abnormal plant behavior early and anticipate potential future problems.

\subsubsection{Response Planning}

Response planning refers to deciding on a course of action, given a particular situation assessment. In general, response planning involves identifying goals, generating one or more alternative response plans, evaluating the response plans, and selecting the response plan that best meets the goals identified.

While these are the classic activities associated with response planning, one or more of these steps may be skipped or modified under certain circumstances. In the case of NPP emergencies where there are EOPs that provide predefined response plans, the need to generate a response plan in real-time is largely eliminated. However, as we will show through illustration, some elements of response planning remain to be accomplished. Operators still need to identify appropriate goals based on their own situation assessment, evaluate whether the actions they are taking based on the procedures they are following are sufficient to achieve those goals, and adapt the procedure to the situation if they decide it is necessary.

One cognitive activity included under response planning is monitoring the effectiveness of the response plan embodied in the EOPs. Response plan monitoring includes evaluating the consequences of particular actions specified in the procedure steps, and evaluating the appropriateness of the EOP path for achieving identified goals. As we will show, response plan monitoring enables operators tn catch errors, including errors made by the operators themselves and errors that may be present in the procedures.

Another cognitive activity included under response planning is response plan adaptation. This includes filling in gaps in a procedure, adapting a procedure to the specific situation, and redirecting the procedure path. Adapting procedures includes taking actions that are not stated in the procedure, not taking actions that are stated in the procedure, and taking actions specified in the procedure but changing them in some way (e.g., changing a plant parameter value mentioned in the procedure). In the analysis that follows we will provide several examples of operators adapting procedures to handle cases that could not be fully addressed by following the procedures verbatim.

\subsection{Overview of Emergency Scenarios}

The model described above guided design of emergency scenarios that were expected to be cognitively demanding. Variants of two base scenarios were run: an ISLOCA into the RHR system and a Loss of Heat Sink (LHS) event complicated by a leaking pressurizer PORV. These emergency scenarios were designed to create situations where active situation assessment and response plan evaluation and adaptation were needed on the part of the operating crew to handle the events.

The core characteristics of the scenarios were defined by the project team based on pilot studies previously conducted on the Westinghouse training simulator using training instructors as crew members. Using these base scenarios as a starting point, the project team worked with training instructors at each of the two plants to tailor the events to the individual plants. Differences existed between the plants in procedures, simulator characteristics, and operating and training philosophy. Thus the events run at the two plants, while similar in many respects, differed in several important ways.

Sections 2.3.1 and 2.3.2 provide a brief description of the main features of each scenario. More detailed 
descriptions of the scenarios are provided in Appendix A. 6

\subsubsection{ISLOCA Scenarios}

The ISLOCA scenarios involved a leak from the high pressure Reactor Coolant System (RCS) to the low pressure Residual Heat Removal (RHR) System. In one variant of the event (ISLOCA 1) the RCS leak into the RHR eventually led to an RHR pipe rupture in the Auxiliary Building causing reactor coolant fluid to spill onto the floor of the Auxiliary Building. In the second variant (ISLOCA 2) the event started in the same way; however, the buildup of pressure in the RHR led to a break in the heat exchanger between the RHR system and the Component Cooling Water (CCW) system causing RCS fluid to get into the CCW system. (See Figure 2.3 for a simplified diagram of the systems involved in the ISLOCA scenarios. ${ }^{7}$ )

The ISLOCA scenarios were designed to be difficult from the point of view of situation assessment. The objective was to create a situation where the crews had to identify and isolate the leak into the RHR without explicit procedural guidance.

While the EOPs contain procedures for identifying and isolating an ISLOCA, it was possible to create a situation where the crews could not reach the ISLOCA procedure within the EOP network. This is because the plant symptoms generated early in the event are similar to the pattern of symptoms that would be produced by a Loss of Coolant Accident (LOCA) inside containment. By timing the dynamics of the event carefully we were able to create a situation where the EOPs directed the operators to a procedure for a LOCA inside containment.

At Plant 1 in one variant of the event, ISLOCA 1, once in the LOCA procedure there was no explicit transition to the ISLOCA procedure. The crews

${ }^{6}$ The overview of the scenarios presented in Sections 2.3.1 and 2.3.2 is simplified. Because the simplifications are necessarily abstract, readers who are familiar with NPP design and terminology might prefer to read Appendix $A$ at this time.

${ }^{7}$ Figure 2.3 is a highly simplified diagram. A more complete and accurate diagram of the RHR system is provided in Figure A.1 in Appendix A. eventually reached a step in the LOCA procedure that asked them to "try and identify and isolate the leakage." Thus we were able to observe crew performance in a situation where the EOP explicitly required the crews to identify and isolate the leak without more detailed procedural guidance.

At Plant 2 in the second variant of the event, ISLOCA 2 , while there was an explicit transition to the ISLOCA procedure from the LOCA procedure, either the transition step could not be reached, or the criteria for transitioning to the ISLOCA procedure were not met when the transition step was reached. Thus we were able to observe crew performance in a situation where the procedure containing relevant guidance could not be reached within the EOP transition network.

In both variants of the scenario the crews had to identify the ISLOCA into the RHR in attempting to isolate the leak. This situation assessment was cognitively demanding because initial symptoms were typical of a LOCA inside containment. Correct situation assessment required integrating multiple symptoms across different systems. The first alarms indicate pressure and level decreases in the pressurizer. These are soon followed by alarms indicating radiation inside containment. Radiation in containment strongly points to an RCS leak directly into containment (i.e., a LOCA). In fact, the radiation in containment is caused by the leak into the RHR. A relief valve in the RHR system vents to the Pressurizer Relief Tank (PRT) inside containment. The PRT eventually ruptures, resulting in radiation in containment. The crews needed to recognize these physical system interconnections in order to link the symptoms in containment with a potential problem in the RHR.

In ISLOCA 1 a correct situation assessment required the crews to connect the symptoms in containment with the symptoms in the Auxiliary Building. ISLOCA 2 was cognitively more demanding because it required the crews to integrate evidence across more systems and postulate a more complex causal chain of events to account for all the symptoms observed. In particular, the crews needed to recognize that the radiation in the $\mathrm{CCW}$ was due to RCS fluid that leaked into the RHR and entered the $C C W$ via a heat exchanger between the RHR and the CCW. 


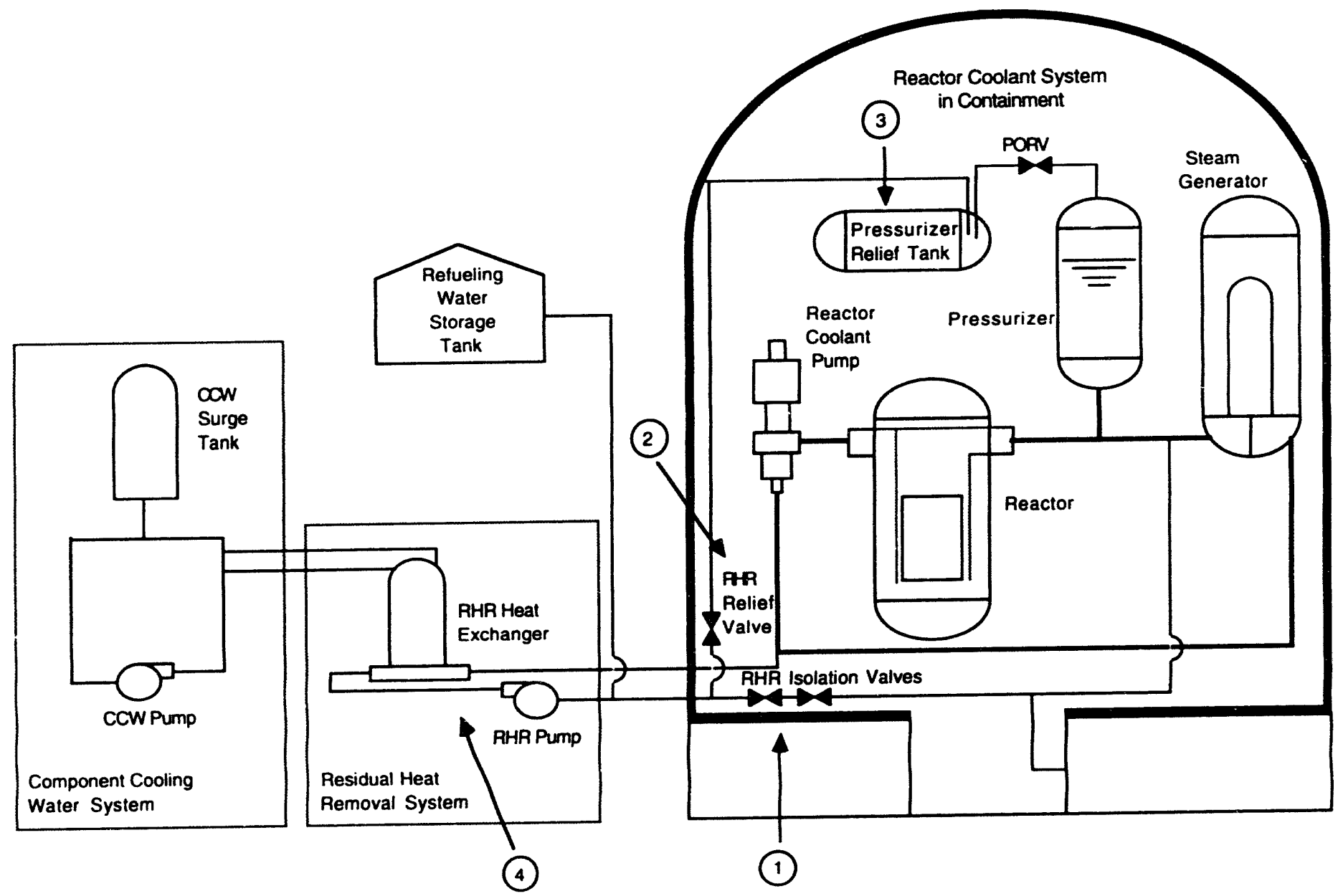

Simplified diagram of the systems relevant to the ISLOCA scenarios. (1) Two RHR isolation valves began to leak. (2) The RHR relief valve opened, directing radioactive fluid to the Pressurizer Relief Tank. (3) The Pressurizer Relief Tunk broke, resulting in radiation symptoms inside containment. (4) Eventually a break occurred in the RHR system. In ISLOCA 1 the break was in an RHR pipe in the Auxiliary Building. In ISLOCA 2 the break was in the RHR heat exchanger with the CCW system. 
Once the operators identified a leak into the RHR they needed to take action to attempt to isolate the leak. The appropriate action to take depended on the postulated source of the leak. In the event we ran there were two hypotheses for the source of the leak that were equally plausible in that they could fully explain the available evidence. One was a failure of the two isolation valves between the hot leg loop of the RCS system and the RHR on the suction side of the RHR pump. This is the event that we postulated. Given this hypothesis the actions required to isolate the leak are to call the Auxiliary Building to request that the valves be re-energized, to verify that they are closed, and to close them if they are not. The alternative hypothesis was that there was a leak back from the RCS through a series of failed check valves. Given this hypothesis, the leak could be isolated by closing an isolation valve on the discharge side of the RHR pump that is normally kept open. 8

In ISLOCA 2 the crews also needed to take action to isolate the leak from the RHR into the CCW. This step required that they identify the RHR heat exchanger as the source of the leak and take action to isolate it.

\subsubsection{Loss of Heat Sink Scenarios}

The Loss of Heat Sink event involved a total loss of feedwater flow complicated by a leaking pressurizer power operated relief valve (PORV). The objective was to create a situation where the EOPs focused operator attention on one high priority problem -- a loss of heat sink -- and then examine how the crews discovered and dealt with a second potentially serious fault that arose: a leaking pressurizer PORV.

The Loss of Heat Sink event was designed to be cognitively demanding from the perspective of both situation assessment and response planning. In this scenario feedwater to the steam generators is lost and the EOPs direct the operators to a Loss of Heat Sink procedure that specifies actions the operators should take in attempting to recover feedwater. While following the Loss of Heat Sink procedure, the operators are directed to open and then close the pressurizer PORV in order to reduce pressurizer

\footnotetext{
${ }^{8}$ The location of the RHR discharge-side check valves and isolation valves are shown in Figure A.1 in Appendix A.
}

pressure. In the event we ran the pressurizer PORV never fully closes (although it read closed), resulting in a leak on the primary side. The analysis focused on how the operators discovered and dealt with the leaking PORV, given that the EOPs provided no explicit guidance. (See Figure 2.4 for a simplified diagram of the systems involved in the Loss of Heat Sink scenarios.)

The scenario was demanding from the perspective of situation assessment because it created a situation where operator judgment was needed to discriminate plant behavior that was the result of known factors (i.e., an operator induced cooldown) from plant behavior that signaled an additional plant fault. Many of the early symptoms of the leaking pressurizer PORV (i.e., decreasing pressurizer level and pressure) could be attributed to a cooldown caused by the control actions that the operators were taking to recover the secondary side heat sink. As the event progressed the symptoms on the primary side became more severe (i.e., reactor vessel level decreased; a bubble formed in the vessel; the pressurizer became solid). Those symptoms could not be explained by a cooldown caused by activities on the secondary side.

The Loss of Heat Sink scenario was also designed to be challenging from the perspective of response planning. In one variant of the scenario, LHS 1 , at Plant 1, secondary side feedwater is never recovered. As a result the crews remain in the Loss of Heat Sink procedure. This variant was designed to place crews in a situation where they had to decide whether to manually initiate a safety system under conditions where procedural guidance was minimal, and multiple goals needed to be considered and balanced.

Specifically, the crews had to decide whether to manually initiate safety injection (SI). There was a step early in the Loss of Heat Sink procedure that had the crews block SI. ${ }^{9}$ This action has potentially serious safety consequences because it means that a major automatic safety actuation system is no longer in operation and must be manually initiated if needed. The only procedural guidance available to the operators regarding manual initiation of SI was in a

\footnotetext{
${ }^{9} \mathrm{SI}$ is blocked to avoid spurious activation of safety injection when the steam generators are depressurized below an SI actuation set point later in the procedure.
} 


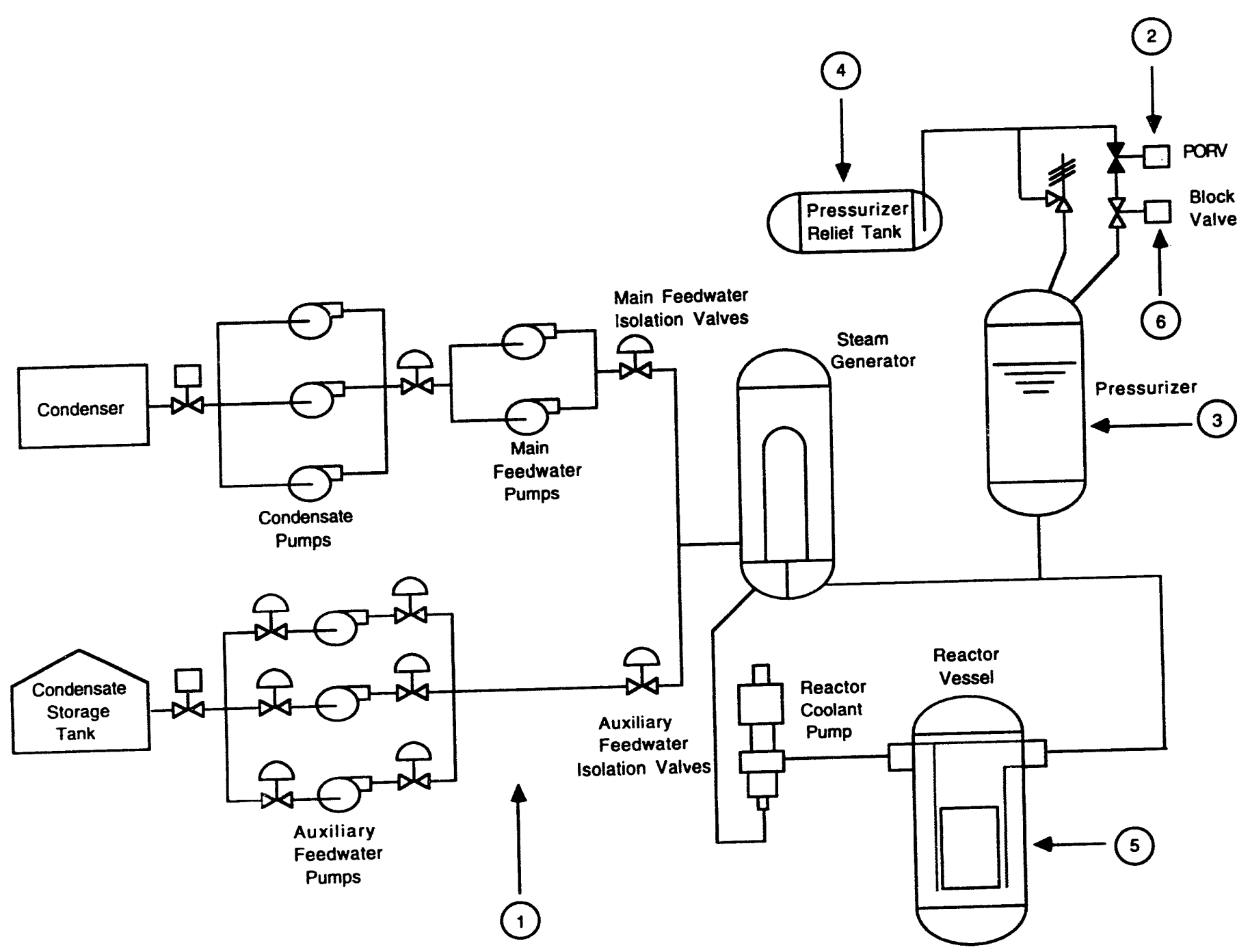

(1) In the LHS event there was a loss of Simplified diagram of the systems relevant the the closed but both main and auxiliary feedwater. (2) As part of a step in the EOP (4) The Pressurizer Relief Tank begins to fill and begins to leak. (3) Pressure and level in the pressurizer begin to fall. (4) The The leak through the PORV can be terminated by eventually breaks. (5) Even closing the block valve. 
Study Methodology

caution that stated: "Following block of automatic SI actuation, manual SI actuation may be required if conditions degrade."

The LHS scenario was designed to place the crews in a situation where they had to decide whether to initiate SI under conditions where there were multiple goals that needed to be considered. The leaking pressurizer PORV created a situation where RCS conditions became progressively more abnormal. Eventually, RCS pressure decreased to the point where a bubble formed in the reactor vessel. Level in the reactor vessel continued downward, while level in the pressurizer started to go up. In some cases the pressurizer became full. The degrading RCS conditions could be mitigated by manually initiating SI; however, the decision of whether to manually initiate SI is made complex because it affects heat sink recovery efforts. Initiating SI would impede efforts to recover feedwater flow on the secondary side, and increase the probability that the crews would have to resort to a less desirable means of achieving a heat sink (i.e., bleed and feed). The objective of this aspect of the scenario was to examine how crews responded to the degrading conditions in the RCS, given that the only relevant procedural guidance available to them was in a caution. Specifically, the analysis focused on whether the crews chose to initiate SI and the rationale for their decision.

The second variant of the Loss of Heat Sink event, LHS 2, at Plant 2, was also demanding from the perspective of response planning. In this scenario the crews eventually got feedwater back. As a result the Loss of Heat Sink procedure transitioned them back to the procedure they had been in when feedwater was lost, which was the Reactor Trip Response procedure. This transition introduced new cognitive challenges because some of the steps in the Reactor Trip Response procedure were no longer appropriate. The crews were now feeding through the condensate system which involves a different plant configuration than is assumed by the Reactor Trip Response procedure. Some of the steps in the Reactor Trip Response procedure, if followed verbatim, would undo actions that had been performed to recover feedwater, causing a lose of heat sink. This variant of the Loss of Heat Sink scenario provided the opportunity to observe how operators respond in cases where actions specified in procedure steps are not perceived to be appropriate to the specific situation. ${ }^{10}$

\subsection{Data Collection and Analysis}

\subsubsection{Participants}

Crews from two NPP sites participated in the study. The two plants were pressurized water reactor (PWR) plants. These will be referred to as Plant 1 and Plant 2. The ISLOCA and Loss of Heat Sink scenarios were run as part of the regularly scheduled requalification training at these plants. As a result all the crews undergoing requalification training at the two plants during that period participated in the study. This included both actual operator crews currently on shift as well as crews composed of administrative staff undergoing requalification training to maintain their Reactor Operator (RO) or Senior Reactor Operator (SRO) licenses.

A total of 11 crews from Plant 1 (five shift crews and six staff crews ${ }^{11}$ ) and 11 crews from Plant 2 (six shift crews and five staff crews) were included in the study.

A full complement crew included five members:

\footnotetext{
10 The developers of the EOPs recognized that the type of situation created in LHS 2, where crews return to a procedure that includes steps that are not appropriate to the situation, could arise. The Users Guide for the Westinghouse Owners Group Emergency Response Guidelines and Background Document explicitly addresses the type of situation created in LHS 2. It states "After restoration of any Critical Safety Function from a RED or ORANGE condition, recovery actions may continue when the FRG is complete... Upon continuation of recovery actions, some judgment is required by the operator to avoid inadvertent reinstatement of a RED or ORANGE condition by undoing some critical step in a Function Restoration Guideline." (Westinghouse Owners Group Emergency Response Guidelines, Users Guide for Emergency Response Guidelines and Background Documents, September 1, 1983, pg. 17.) The use of the phrase "some judgment is required by the operator" suggests that the developers of the EOPs recognize that in these circumstances operators need to evaluate the appropriateness of certain procedure steps based on their own situation assessment.
}

11 Videotapes were collected for two additional crews at Plant 1; however there were technical problems with the quality of the videotapes which prevented the performance of these crews to be included in the study. 
- The Supervising Operator (SO) responsible for reading the procedures and directing and coordinating operator activity;

- The Reactor Operator (RO) responsible for monitoring and control actions on the reactor side;

- The Balance of Plant Operator (BOP) responsible for monitoring and control actions on the balance of plant side;

- The Shift Supervisor (SS), the most senior management level staff person on the scene, responsible for declaring site emergencies, and approving actions;

- The Shift Technical Advisor (STA) responsible for monitoring the status trees and providing technical advice.

In several cases four-member crews were run with one person simultaneously taking on the SS and STA roles.

In order to preserve the anonymity of the crews, each crew was assigned an arbitrary letter (at Plant 1) or number (at Plant 2) to serve as identifier. These were used as crew identifiers in the transcribed protocols, and in the presentation of results in Section 3.

\subsubsection{Procedure}

Two scenarios (one variant of an ISLOCA and one variant of a Loss of Heat Sink) were run by the training instructors at each plant as part of the regularly scheduled requalification training conducted on a high fidelity training simulator. Crew members did not have prior knowledge of the event to be run.

Instructions were prepared to be read by the training instructor to the crews at the beginning of the session. Appendix B contains a copy of these instructions.

Briefly, the instructions informed the crews that the two events they would be participating in were part of a research project being conducted by the Westinghouse Science \& Technology Center for the NRC's Research Office. They were told that the purpose of the research is to understand the decisionmaking process involved in diagnosing and responding to challenging emergency scenarios. They were asked to "Handle these events as you would if they were actually happening in the plant. Use all of the resources available to you -- anything you would use in a real situation to mitigate the event." The crews were informed that the session would be videotaped to facili.ate analysis but they would only be viewed by the research team. It was emphasized that this was strictly a research project, that they were not being evaluated, and that every reasonable effort would be made to preserve the anonymity of the crew participants in reporting the results.

Occasionally, one or more of the crew members had prior knowledge of the event to be run. This primarily happened in cases where the training instructor staffs were used to fill out a crew. In those cases the individual was directed to play a passive role, providing information and taking control actions as requested, but not volunteering interpretation or advice, or actively participating in situation assessment discussions. The crews where one or more members were cognizant of the event are explicitly noted in the analysis.

After each event was run a debriefing was conducted. The instructor began by asking whether any crew member had prior knowledge of the event. If so, that was noted. He then reminded them that the event they had participated in was part of a research project and requested that they do not discuss the event with anyone who has not yet participated in the events. The instructor then led a relatively unstructured debriefing similar in format to the type of debriefing conducted during regular simulator training exercises. The instructor had the crew summarize the plant faults they thought were present, actions they decided to take, and the reason for their actions.

The simulator sessions, including the debriefing, were videotaped. The videotapes included a date and time stamp that enabled identification of the time at which key activities took place.

At the end of the session the instructors filled out summary data sheets for each crew on each event run. The summary sheets included background information on the crew members (whether it was a crew on shift or a staff crew, the licenses each crew member held, and their education); indication as to 
which crew members, if any, had prior knowledge of the event; yes/no questions regarding whether key situation assessments and actions were made; and an area where the instructor could write in brief remarks. Examples of the summary sheets are included in Appendix B.

In addition, data tapes were made recording key plant parameters and alarms off of the high fidelity training simulator while the event was running. These data tapes were used as a backup resource to check on plant parameter behavior in cases where questions arose.

\subsubsection{Data Analysis}

The crew performance videotapes constituted the primary source of data in the study. Protocols of the perform ince of each crew on each event run were produced from the videotapes. The protucols consisted of partial transcripts of crew dialogue that documented crew observations, hypotheses, discussions, and actions related to the key faults in the events.

The analysis primarily focused on providing evidence of the role of higher-level cognitive activity in guiding operator performance in the scenarios. The logic employed in the analysis is described below, and captured schematically in Figure 2.5.

The protocols document observable behavior -- either actions or utterances. Some examples are provided in Figure 2.5. These include monitoring plant parameters, interpreting plant state, taking a control action, reviewing steps in the EOPs, and checking schematics. Two main types of activities were observed:

(1) Behaviors that were directly the result of following steps in the EOPs. These are referred to as procedure-driven activities, and were not analyzed further.

(2) Other behaviors not directed by the specific step in the EOP that the crew was following at that point in time. These behaviors are referred to as extra-procedural activities..
In Figure 2.5 procedure-driven activities appear without a border and extra-procedural activities appear inside a rectangular border.

Extra-procedural activities served as the primary focus of analysis. Situations where extra-procedural activities arose were examined to:

(1) Identify the kinds of situations that arose in the scenarios where extra-procedural activities were required to deal with the situation; and

(2) Obtain evidence that the crews were engaging in situation assessment and response planning in these situations.

The model of cognitive activities presented in Section 2.2 provided the framework for inferring higher level cognitive activities from specific extra-procedural activities. The model specified types of observable behavior that result from situation assessment and response planning (see Figure 2.2). The specific extra-procedural activities documented in the protocols were examined to determine if they represented instances of these types of behavior. Examples include cases where operators monitored a plant parameter to obtain evidence to confirm a hypothesis, or to search for an explanation for an unaccounted plant behavior. These documented cases were then used as evidence of the higher-level cognitive activity. Figure 2.5 illustrates the link between specific observed instances of extraprocedural activities, the type of behavior they exemplified, and the higher-level cognitive activity for which they provided evidence. For example, cases where operators monitored a plant parameter to confirm a hypothesis were used as evidence of situation assessment.

\subsubsection{Information Recorded in the Protocol}

The objective of the protocol was to document the observations made, hypotheses considered, situation assessments made, and actions taken related to identifying and responding to the faults in the scenario. Particular attention was paid to documenting instances where operators summarized their situation assessment; engaged in extraprocedural activity; reflected on the goals or objectives of the procedures; made judge.ent calls; or modified a procedure step. 


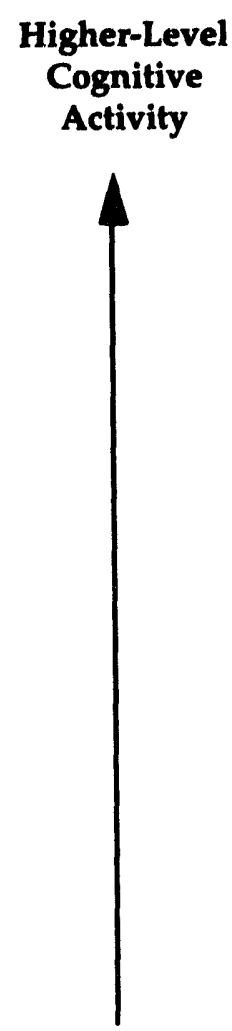

Observable
Behavior:

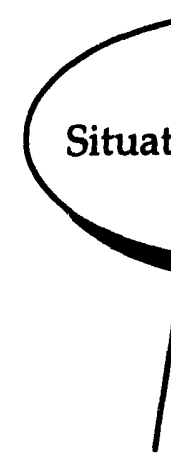

Check for

Evidence to Confirm Hypothesis

Identify Problems (e.g., sensor failures; plant malfunctions)

Explanation

Search for
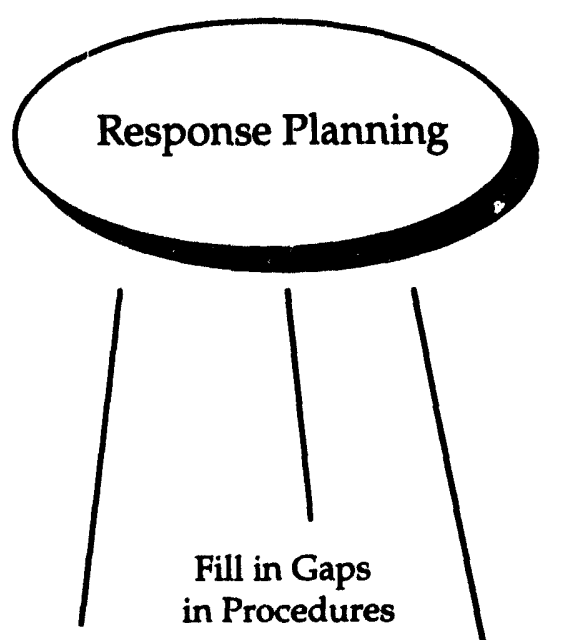

Evaluate Appropriateness of EOP Procedural Path
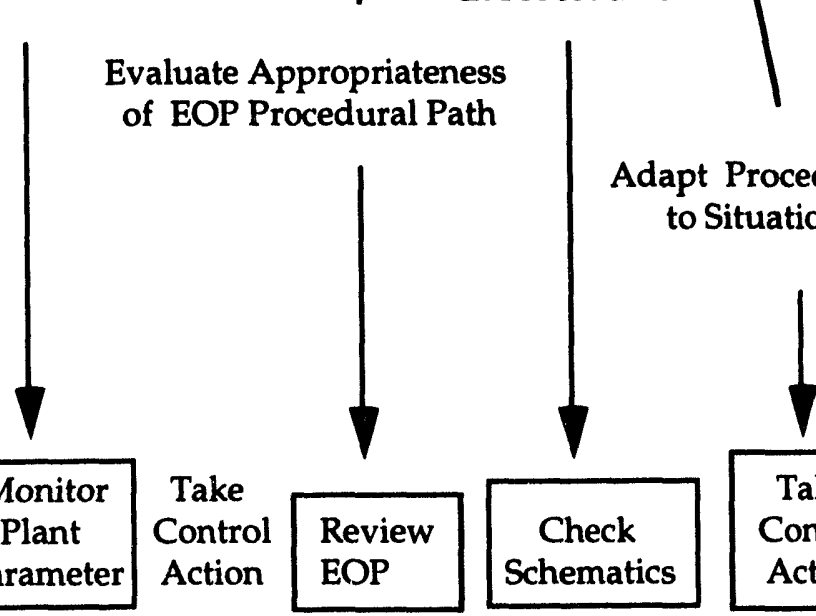

Adapt Procedures to Situation

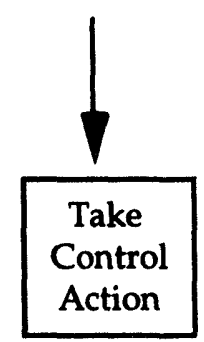

Figure 2.5 Illustration of the logic employed to infer situation assessment and response planning from the observable behaviors documented in the protocols. Specific observable behaviors - either actions or utterances - were classified as procedure-driven (no border) or extra-procedural (a rectangular border). The extra-procedural activities were examined to determine if they represented instances of observable behaviors that result from situation assessment and response planning (see Figure 2.2). In this way a link was established between specific observed instances of extra-procedural activities, the type of behavior they exemplified, and the higher-level cognitive activity for which they provided evidence.

Activities recorded in the protocol included:

- Observation of alarms, plant parameter values, and automatic system actuation (e.g., reactor trip, SI; pressurizer pressure and level; PRT symptoms; RHR symptoms; containment symptoms);
- Key procedure steps (e.g., steps that resulted in procedure transitions; steps that resulted in loop back to earlier steps; steps that required judgment calls; steps that required operators to engage in diagnosis and response planning; key cautions); 
- Hypotheses considered;

- Actions taken or considered related to dealing with the faults (e.g., closing isolation valves; closing block valves; manual SI);

- Information that is volunteered or requested that is not strictly called for by the procedure;

- Cases where operators are performing activities not called out in procedures (e.g., reviewing schematics);

- Discussions among staff regarding the interpretation of the situation or appropriate actions to be taken;

- Instances where someone "recapitulates" the situation as they understand it;

- Instances where someone requests opinions or consensus about the situation assessment or actions to be taken;

- Discussions regarding the EOPs and their appropriateness to the situation (e.g., whether the procedures will eventually get them to where they want to be; whether other procedures should be consulted).

For each of these cases the protocol documented the time it occurred, who was involved (SO, RO, BOP, STA or SS), and a verbatim transcription or very close paraphrase of what was said. Statements that are not verbatim transcriptions are presented in parentheses or between stars (e.g., ${ }^{* * *}$ The SO asked the STA to check the status trees ${ }^{* * *}$ ). To preserve the anonymity of the crew members, in cases where crew members were referred to by name, their crew position was substituted for the name. In cases where the individual making the statement could not be identified, question marks were used in place of crew position.

Note that all conclusions about plant symptoms observed, conclusions drawn, and actions taken are derived from analysis of crew dialogue during the videotaped scenarios and the debriefing. If a crew member noticed a parameter, or took an action, but did not mention it either during the event or as part of the debriefing, then we had no evidence from the dialogue that the behavior occurred, and assumed that the behavior did not occur. In this sense, the protocols and analysis are based on the externally observable distributed group cognition (Hutchins, 1990; 1991).

\subsubsection{Behaviorally Anchored Rating Scales (BARS) of Team Interaction Skills}

Based on the videotapes of crew performance, ratings were made of team interaction skills using the BARS scales developed by Moritgomery et al. (1992). This rating scale was developed under the sponsorship of the U.S. Nuclear Regulatory Commission specifically to support evaluation of NPP crew team interaction skills.

In the BARS methodology crew interaction skills are evaluated on each of six dimensions:

- Communications

- Openness

- Task coordination

- Team spirit

- Maintaining task focus in transitions

- Adaptability

Montgomery et al. (1992) define each of these dimensions as follows:

- The communications dimension consists of the transmission of factual information in a clear and concise manner. In terms of crew behaviors this includes listening skills, nonverbal behavior, and articulation of plant status or instructions for future activities. Communication does not include emotional aspects of information transmission.

- Openness consists of crew members' tendency to ask for, give, and receive suggestions. It includes questioning decisions and discussing alternatives to arrive at the best possible decision. Openness also involves the reactions of crew members to feedback, which should focus on the task rather than on the person when reviewing actions.

- Task coordination refers to the crew members' ability to match the available resources, such as people and procedures, to the task in order to achieve the optimal workload distribution. 
- Team spirit consists of mutual support, cohesiveness, group identity, and the extra effort that crew members exhibit to accomplish a common goal.

- Maintaining task focus in transitions deals with crew members' responses to changes from normal to non-normal plant conditions (e.g., loss of pressure in feedwater pump). These responses include focusing on the task, avoiding emotional overreaction or panic, and maintaining poise.

- Adaptability reflects crew members' ability to adjust or modify their behavior based on the situation, to be flexible in responding to the environment, and to recognize the need for change.

Performance on each of these dimensions is rated on a seven point scale where $\mathrm{a}$ is low (poor) and 7 is high (good). Copies of the BARS rating scales are presented in Appendix C.

The ratings were performed by a single individual (the first author) who also prepared the protocols from the videotapes. The ratings for each crew on each scenario are presented in Section 4 on team performance. 


\section{Cognitive Performance in the Simulated Emergencies}

This section presents the main results for operator cognitive performance in the four simulated emergency scenarios. The results for each scenario are organized around cases where extra-procedural activity was needed to handle the situation. In each case we examined the extra-procedural activities observed for supporting evidence of situation assessment and response planning as described.

The data presented include tables that summarize performance across crews on particular aspects of the scenario. These tables indicate the consistency and variability in performance among crews.

In addition, protocol segments for particular crews are presented that cover a more extensive portion of the scenario. These protocol segments illustrate the complexity of the situations the crews confronted and the role that situation assessment and response planning played in guiding crew performance.

\subsection{ISLOCA 1: ISLOCA into RHR Leading to Pipe Rupture in Auxiliary Building}

\subsubsection{Summary of Simulated Scenario}

Analysis of crew performance in ISLOCA 1 identified two cases where operators had to engage in situation assessment and response planning to deal with the situation. The first case is the situation around which the ISLOCA 1 scenario was designed: a case where a step in the EOP explicitly requests the crews to identify and isolate a leak, without providing more detailed procedural guidance. Analysis of the extraprocedural activities the crews engaged in to identify and isolate the ISLOCA into the RHR. provided specific, concrete examples of the types of situation assessment and response planning behaviors which are listed in Figure 2.2.

The second case is a specific example of a situation where operators need to engage in situation assessment in order to determine how to respond to a procedure step. The specific case arose in a step in the
LOCA procedure that they were following. ${ }^{12}$ The step asks whether pressure in all steam generators is stable or increasing. The purpose of this step is to check for the possibility of a faulted steam generator (SG), which would cause SG pressures to decrease. ${ }^{13}$ In fact, the SG was not faulted, but there was a cooldown in progress, which also results in steam generator pressures decreasing. Based on training and EOP background documents, crews are instructed that if they are taking actions that are producing a cooldown they should consider a decrease in SG pressure to be "stable or increasing." Thus, this is a case where response to a procedure step is based on situation assessment rather than literal interpretation of the procedure step. In deciding how to respond to the procedure step they need to assess whether the plant behavior they are observing is the result of known manual and/or automatic actions that are producing a cooldown, or the result of a plant fault. The analysis focused on how crews responded in cases where SG pressures were decreasing when they got to this step.

\subsubsection{Characteristics of Participating Crews}

Eleven crews from Plant 1 participated in the event. Of these, five were crews currently on shift and six were staff crews made up of administration personnel. Crew size ranged from four to five people. In four cases one or more of the crew members had prior knowledge of the event. Those individuals did not actively participate in situation assessment and response planning. ${ }^{14}$

\footnotetext{
12 The actual title of the LOCA procedure is "The Loss of Reactor or Secondary Coolant Procedure," which is also referred to as E-1. In this report, LOCA procedure, Loss of Reactor or Secondary Coolant procedure, and E-1 are used synonomously.

${ }^{13} \mathrm{~A}$ faulted steam generator is defined as having a discontinuity in the pressure boundary allowing either steam or feedwater to leak out. Examples are steam line breaks and feed line breaks.

14 The crews that included individuals with prior knowledge of the event were: Crew B (the STA), Crew D (the SS), Crew L (the SS and STA), and Crew G (the STA).
} 


\subsubsection{A Case Where a Step in the EOP Explicitly Requests the Crews to Identify and Isolate a Leak}

The ISLOCA scenario was timed so that containment symptoms occurred early in the event. 15 Given containment symptoms, the EOP directed the operators to the LOCA procedure. Once in the LOCA procedure there was no explicit procedure transition to the ISLOCA procedure. ${ }^{16} \mathrm{~A}$ step in the LOCA procedure checked for radiation in the Auxiliary Building. If there was radiation, which is an indicator of an ISLOCA, the step said to: "Identify and isolate the leakage."

By the time the operators reached that step in the LOCA procedure, the RHR piping had ruptured, resulting in radiation in the Auxiliary Building. This enabled us to observe crew performance in a situation where the EOP explicitly required the crews to identify and isolate a leak without providing more detailed procedural guidance.

The data analysis focused on the activities the crews engaged in to identify and isolate the ISLOCA into the RHR. Specifically:

- Whether crews detected symptoms of a problem in the RHR system;

- Whether crews identified a problem in the RHR system, and if so on what basis;

- How crews explained plant symptoms they observed;

- Whether crews recognized that the symptoms inside and outside containment all resulted from

\footnotetext{
15 There were symptoms inside containment because the RHR system includes a relief valve that vents to the PRT, which is inside containment. When pressure in the RHR increased, the relief valve opened and radioactive fluid was directed to the PRT. The PRT ruptured causing radiation alarms within containment.

${ }^{16}$ It should be noted that, while at the particular time that we ran this event, there was no explicit transition from the LOCA procedure to the ISLOCA procedure in the EOPs used at that plant, the latest version of the Emergency Response Guidelines (ERGs) does include an explicit transition from the LOCA procedure to the ISLOCA procedure.
}

the same underlying fault, and if so on what basis;

- Actions that the crews took to identify and isolate the ISLOCA, given that they had no explicit EOP guidance in performing this task.

This section describes the extra-procedural activities the crews engaged in to identify and isolate the ISLOCA into the RHR. These activities are specific, concreie examples of the types of situation assessment and response planning behaviors which are listed in Figure 2.2.

\section{Situation Assessment: Detecting abnormal RHR symptoms}

The analysis examined whether crews detected symptoms of a problem in the RHR system. Since the EOPs did not explicitly direct the crews to check for RHR symptoms, identification of RHR symptoms was based on situation assessment activities.

The first symptom of ar RHR problem was an RHR discharge pressure high alarm that came in prior to the reactor trip. Meters were also available on the control board that, if the crews checked, provided symptoms of a problem in the RHR system (i.e., high RHR discharge pressure and high RHR discharge temperature).

Not all the crews detected the RHR high discharge pressure alarm when it came in. ${ }^{17}$ Six of the 11 crews were not aware that an RHR alarm came on. ${ }^{18}$ The fact that six of the crews did not know about the RHR alarm provided the opportunity to look at the

${ }^{17}$ Among reasons for the difference in detection is that, because of simulator characteristics, the event did not always run in exactly the same way. Sometimes the RHR alarm sounded up to 30 seconds prior to the reactor trip, whereas other times it came within 10 seconds of the reactor trip.

${ }^{18}$ There are two points to note with respect to detection of the RHR alarm. First, that alarms will not necessarily be noticed if they are embedded in a large set of alarms as occurred here. Five of the 11 crews did not mention outloud that there was an RHR alarm at the time it came on. A related point is that, even if an alarm is called out, it may be forgotten during the event. In one case (Crew G), although someone on the crew called out the alarm when it came on, the cause of the alarm was not pursued. In the debriefing everyone on the crew claimed to have been completely unaware of the alarm. 
difference in performance between crews that noticed the RHR alarm early, and crews that did not. As will be seen the performance of these two groups varied in significant ways.

One of the first differences to be noted is whether the crews checked for RHR symptoms on the control board (i.e., RHR discharge pressure and RHR discharge temperature). All five crews who detected the RHR alarm checked RHR control board parameters and noticed abnormal RHR parameter behavior. In contrast, five of the six crews who did not know about the RHR alarm, failed to detect the RHR symptoms. The search for RHR symptoms provides a clear instance of knowledge-driven monitoring that leads to the identification of abnormal plant behavior. In this case, the crews had no procedural guidance to check RHR symptoms. They checked them because the RHR alarm led them to suspect an RHR problem.

\section{Situation Assessment: Delayed detection of alarms/symptoms}

One crew (Crew E) provided a concrete example of how a situation assessment can aid in detecting alarms and symptoms that were missed earlier. This crew had not noticed the RHR symptoms early in the event. The crew decided to check for RHR symptoms late in the event, after they were informed by a call from the Auxiliary Building operator that there was a problem in the RHR. By that point the primary indicator in the control room of an RHR problem, RHR discharge pressure high, was no longer present because RHR discharge pressure decreased when the RHR pipe in the Auxiliary Building broke. The crew decided to examine the alarm printout. By reviewing the alarm printout they were able to identify that an RHR discharge pressure high alarm had occurred prior to the reactor trip. This case provides a concrete example of how searching for an explanation for unexplained plant behavior can result in the detection of alarms that were previously missed.

\section{Situation Assessment: Identifying a problem in the RHR}

The analysis examined whether crews identified a problem in the RHR system, and if so on what basis. Crews that had detected the RHR alarm identified the problem in the RHR earlier than crews that had not.
The contrast in performance between these two groups provided a concrete example of the value of knowledge-driven monitoring and situation assessment in identifying problems early.

A difference existed between the crews that had initially observed the RHR alarm and those that had not. Four of the five crews that had initially observed the RHR alarm identified a problem in the RHR early based on the RHR alarm or the RHR discharge pressure control board readings. In contrast, of the six crews that were not aware of the RHR alarm, four did not identify the RHR problem until they received a call from an instructor, playing the role of an Auxiliary Building operator, telling them that there was an RHR problem (in the case of Crew E), or providing a strong clue to that effect (i.e., telling them that there was radioactive fluid outside the RHR pump room).

The remaining two crews that had not noticed the RHR alarm (Crews J and L) were never told about the radioactive fluid outside the RHR pump room. For these crews the only evidence pointing to an RHR problem was the increase in PRT level and subsequent break in the PRT. Of the two crews in that situation, one crew (Crew $L$ ) never localized the RHR problem. The other crew (Crew J) localized the problem by pursuing sources of input into the PRT, and then checking the alarm printout. This latter crew provided an excellent example of how a crew utilizes knowledge-driven monitoring and external resources, in this case the alarm print out, to form a situation assessment, localize a fault, and determine a course of action. The performance of this crew will be examined in more detail below.

The contrast in performance between the crews that detected the RHR alarm early and those that did not illustrates the value of knowledge-driven monitoring and situation assessment. The crews that detected the RHR alarm identified a problem in the RHR earlier in the cvent than the crews that required symptoms of a burst RHR pipe in the Auxiliary Building before identifying the RHR problem. In an actual ISLOCA incident, early detection of a problem in the RHR would be important, because it would provide the potential for isolating the leak into the RHR before the RHR piping burst. Once the RHR pipe burst the ISLOCA became unisolatable. 


\section{Situation Assessment: Explaining radiation in containment}

The analysis examined how crews explained observed symptoms, and how situation assessment affected explanation of symptoms.

Early plant symptoms included high temperature and pressure in the PRT, the PRT rupturing, and radiation in containment. The containment radiation was caused by the rupture of the PRT, which resulted in release of radioactive fluid into containment. We examined whether crews observed the PRT and containment symptoms, and whether they linked the containment symptoms to the rupturing of the PRT.

Ten of the 11 crews noticed the high temperature and pressure PRT alarms prior to the reactor trip. Later in the event the PRT ruptured. Of the ten crews that noticed the PRT alarms, nine mentioned the PRT rupturing at some point in the event. Only one crew (Crew M) showed no indication of being aware of the PRT symptoms and rupture.

When containment symptoms arose, six of the 11 crews attributed the containment symptoms to the fact that the PRT had ruptured. ${ }^{19}$ The remaining crews gave no verbal indication of how they explained the containment symptoms. Some of these may have also attributed the containment symptoms to the PRT rupture without verbalizing it.

The fact that the majority of crews recognized the source of radiation in containment illustrates the role of situation assessment in explaining observed symptoms.

\section{Situation Assessment: Requesting a search and explanation for symptoms in the Auxiliary Building}

Containment radiation provided a concrete example of a symptom that could be explained by the operators' situation assessment. In contrast, radiation in the Auxiliary Building provided an example of where an unexplained symptom triggered extraprocedural activity in search of an explanation.

19 One of the crews (Crew B) correctly attributed the containment symptoms to the rupturing of the PRT, but attributed the PRT symptoms to a PRZR steam space leak.
Ten of the 11 crews identified the Auxiliary Building problem based on the Auxiliary Building alarms which came in early in the event, before they reached the LOCA procedure. 20 of those ten crews, five called the Auxiliary Building operator to ask them to search for possible sources of RCS leak into the Auxiliary Building. This action was not directed by the procedure the operators were following. The call to the Auxiliary Building operator illustrates extraprocedural activity in search of an explanation for an unexplained plant symptom. In this case they were trying to obtain information to aid them in localizing the source of the leak into the Auxiliary Building. There is an EOP procedure step that explicitly asks crews to check for Auxiliary Building symptoms, but that step occurs later in the EOPs. Calling the Auxiliary Building when the alarm is received, while not based on an explicit procedure step, is considered good practice based on training and standard practice. This extra-procedural action provides another illustration of the role of situation assessment activities in enabling crews to detect and pursue plant symptoms earlier in the event than would otherwise be possible.

Situation Assessment: Searching for an explanation of symptoms both inside and outside containment

To isolate the leak into the RHR, the crews had to identify the source of the leak. This required active situation assessment. We examined how crews explained the symptoms in the RHR and whether they recognized that the symptoms inside and outside containment all resulted from the same underlying fault. The case provided a concrete example of an active search for an explanation that linked multiple symptoms from diverse systems.

Table 3.1 presents the hypotheses that were considered by the crews to explain the symptoms observed inside and outside containment. In these tables 'LOCA' refers to a loss of coolant accident inside containment; 'check valve' refers to the hypothesis that the RHR symptoms were caused by a leak back through a series of check valves.

\footnotetext{
20 The Auxiliary Building alarms came in while the crews were in the entry EOP procedure, which is the Reactor Trip or Safety Injection procedure, also called E-0.
} 
Table 3.1 ISLOCA 1. Hypothesized explanations for plant symptoms.

\begin{tabular}{cccc}
\hline Crew No. & First Hypothesis & Revised Hypothesis & Final Hypothesis \\
\hline A & check valves & no; leak terminated & check valves \\
B & steam space leak and SI ISLOCA & RHR ISLOCA & check valves \\
C & RHR ISLOCA & check valves & check valves \\
D & RHR ISLOCA & isol. or check valves & isol. or check valves \\
E & LOCA and letdown line break & check valves & check valves \\
F & RHR ISLOCA & check valves & isol. or check valves \\
G & RWST to RHR to aux. floor & no & RHR problem \\
H & RHR ISLOCA & check valves & check valves \\
J & RCP seal leak offs & check valves & isol. or check valves \\
L & LOCA and ISLOCA & no & two leaks - LOCA and ISLOCA \\
M & RHR check valves & Unisol. check valve & two leaks - check valve and small LOCA \\
\hline
\end{tabular}

Initial hypotheses were varied and included several cases where crews postulated two separate hypotheses (Crews E, L and B) to explain the symptoms inside and outside containment. By the end of the event most crews correctly identified the RHR problem, and either attributed it to back flow through check valves (five crews) or specifically indicated that it could be either back flow through the check valves or leaking isolation valves between the RCS and the RHR (three crews). These eight crews correctly determined that the RHR problem explained the symptoms in containment as well. The final situation assessment reached by these eight crews can be considered to be as complete, specific, and accurate as would be possible to reach given the evidence presented. 21

\footnotetext{
21 While the check valve hypothesis was not the fault we originally postulated for this scenario, the training instructors felt it was at least as plausible an explanation for the observed symptoms as two leaking isolation valves between the RCS and the RHR systems. In several cases when the crews took action to isolate the check valves (i.e., they isolated the RHR train), the instructors terminated the leak into the RHR; thus in those cases the scenario was run as if the check valves were the source of the leak. Because the scenario was sometimes run in this way, and because in cases where the crews did ask the Auxiliary Building operator to check the two isolation valves between the RCS system and the RHR, they were told the valves read closed, it was decided to consider both hypotheses to be equally valid. A more strict criterion would require crews to have explicitly considered both hypotheses.
}

Of the remaining three crews, one crew, who had originally considered the hypothesis that the radioactive water was coming from the RWST into the RHR, had an unspecific situation assessment of some problem in the RHR by the end of the scenario. The remaining two crews believed that there were two leaks: a LOCA inside containment and an ISLOCA by the end of the scenario.

Several important points should be noted from these results. First, the majority of crews (eight of 11 ), ended with a highly plausible and specific situation assessment to account for the observed symptoms in the Auxiliary Building, in the PRT, and in containment. Second, not all crews were able to recognize that there was a single explanation that could connect all the symptoms. Two of the crews postulated two independent faults, a LOCA inside containment and an ISLOCA, as their final hypotheses. This explanation is less plausible because it assumes two independent faults; further, it does not link the symptoms in the PRT with the symptoms in containment. 22

Another point to be noted regards the process of hypothesis revision and the role that multiple crew members play in revising hypotheses. Five of the 11 crews (Crews E, J, L, G, and B) began with hypotheses

\footnotetext{
${ }^{22}$ The crews that postulated two independent faults did not mention that the PRT had ruptured at any point in the event, suggesting that they may have failed to detoct that the PRT had nuptured.
} 
that were relatively implausible. Of these, three (Crews E, J, and B) eventually revised their hypotheses to a more plausible explanation. In all three cases the crew member who suggested the more plausible hypothesis was different from the crew member who suggested the original hypothesis. This result provides suggestive evidence of the positive contribution of multiple crew members in situation assessment and problem-solving. It is consistent with the argument that has been made by several researchers (e.g., Reason, 1990) that an incorrect situation assessment, is more likely to be corrected by someone new on the scene than by the person who generated the incorrect hypothesis in the first place.

\section{Response Planning: Accessing additional resources}

Since the LOCA procedure provided little direct guidance in localizing and isolating the ISLOCA leak, this case provided a concrete example of a situation where response planning on the part of crew members was required to localize and isolate the leak. We examined what actions crews decided to take based on their situation assessment, and what resources they drew on to support identification and evaluation of candidate actions.

One potential resource was the ISLOCA procedure, which provided step-by-step instruction for opening and closing valves to locate and isolate the ISLOCA. Four of the 11 crews decided to consult the ISLOCA procedure for guidance. Note that these crews had to actively decide to consult the ISLOCA procedure. There was no explicit EOP transition to the ISLOCA procedure.

Other resources accessed included schematic prints and alarm printouts. All 11 crews consulted schematic prints to identify potential flow paths from the RCS system into the Auxiliary Building and the RHR system. In addition three of the 11 crews reviewed alarm printouts.

Response Planning: Identifying and evaluating extraprocedural response actions

Since the LOCA procedure provided no specific guidance on the actions that should be taken, this case provided a concrete example of identifying and evaluating potential mitigating actions based on situation assessment.

Two plausible hypotheses could explain the flow of RCS water into the RHR system. ${ }^{23}$ One hypothesis is that the two isolation valves between the RCS hot leg loop and the suction side of the RHR system are open. Given this hypothesis, the leak into the RHR could be terminated by closing these valves. Seven of the 11 crews mentioned the possibility of these valves being open, but only two crews (Crews $D$ and $H$ ) checked that the valves were open. One of these crews (Crew $\mathrm{H})$ checked the valves as part of following the ISLOCA procedure. In most cases, while the crews considered the possibility that RCS fluid could be coming in through these valves, they rejected it as implausible because the valves were supposed to be closed and de-energized.

Given the hypothesis of back leakage via a series of check valves, a second plausible action for terminating the ISLOCA is to isolate the RHR train from the cold leg side of the RCS by closing a valve on the discharge side of the RHR pump that isolates the RHR system from the cold leg loops. ${ }^{24}$ This valve is normally open. One of the actions called out in the ISLOCA procedure is to close this valve and check if that terminates the leak. Table 3.2a presents data on whether crews considered closing this valve ('consider Isolate RHR train'), which crew member suggested it, and the basis for the suggestion. As can be seen ten of the 11 crews considered closing this valve. A point to note is that in all cases the suggestion to close the valve was based on a situation assessment rather than a particular procedure step.

\footnotetext{
${ }^{23}$ The location of the isolation valves between the RCS hot leg loop and the suction side of the RHR system is shown in Figure A- 1 , in Appendix $A$.

24 The location of the valve on the discharge side of the RHR pump is shown in Figure A-1, in Appendix A.
} 
Table 3.2a ISLOCA 1. Consideration of RHR train isolation. 25,26

\begin{tabular}{cccc}
\hline Crew No. & Consider Isolating RHR Train & Crew Member & Reason Given \\
\hline A & yes & BOP & check valve hypothesis \\
B & yes & SO & RHR problem \\
C & yes & BOP & check valve hypothesis \\
D & yes & BOP & RHR problem \\
E & yes & RO & check valve hypothesis \\
F & yes & RO & RHR problem \\
G & yes & SO, SS & RHR problem \\
H & yes & STA, SS & check valve hypothesis \\
J & yes & RO & check valve hypothesis \\
L & no & n/a & n/a \\
M & yes & SS & RHR problem \\
\hline
\end{tabular}

Table 3.2b ISLOCA 1. Decision to isolate RHR train.

\begin{tabular}{ccc}
\hline Crew No. & Decision & Reason Given \\
\hline A & delay & no procedural guidance \\
B & yes & $\mathrm{n} / \mathrm{a}$ \\
C & yes & $\mathrm{n} / \mathrm{a}$ \\
D & delay & no procedural guidance \\
E & yes & /a \\
F & delay & check train B operable \\
G & delay & use ISLOCA procedure. as guidance \\
H & yes & SO says OK since RHR pumps off \\
J & yes & SO checks with SS and Emergency Duty \\
& & Officer \\
L & n/a & n/a \\
M & yes & can reopen valve if needed \\
\hline
\end{tabular}

${ }^{25}$ SO= Supervising Operator; RO= Reactor Operator; BOP= Balance of Plant Operator; SS= Shift Supervisor; STA= Senior Technical Advisor; $n / a=$ not applicable.

26"RHR problem" indicates that the crews recognized that there was a leak into the RHR but were not more specific with respect to the source of the leak; $n / a=$ not applicable. 
Response Planning: Evaluating consequences of extraprocedural actions

Table $3.2 \mathrm{~b}$ shows whether the crews decided to close the valve that isolates the RHR train, given that it was suggested, and the basis for the decision. As can be seen, six crews decider to close the valve right away, while four crews decided to wait. The basis for the decisions are illuminating in that they reveal the cautiousness of the operators in taking an action not explicitly called out in the EOPs. Of the six crews who decided to close the valve, three articulated the basis of their decision. Of those, the reasons provided by two showed that they checked for potential negative consequences before taking the action. One crew said that the RHR pumps were off, so closing the valves would have no effect. The other crew said that they would be able to reopen the valve if needed. In the case of the third crew, they checked with the SS and the Emergency Duty Officer (EDO) ${ }^{27}$ for approval before taking the action. Similarly, of the four crews who delayed taking the action, in three cases, the reason given was that they had no explicit procedural guidance to take the action. Of those one crew waited until they got to the appropriate step in the ISLOCA procedure before taking the action. The other two eventually decided to close the valve. The fourth crew (Crew F) decided to wait until they checked that RHR train B was operable before they isolated train $\mathrm{A}$.

The decision faced by the crews with respect to whether to isolate the RHR train, provides a concrete example of crews identifying and evaluating a response action. These results show that the majority of the crews were cautious in taking actions that are not explicitly called out in the procedures. While many of the crews did decide to take the action if they felt it could mitigate the problem, they considered several factors before taking the action:

a) Whether the functions performed by the system were currently needed or a need could be foreseen;

b) The reversibility of the action; c) Whether alternative ways to achieve the functions the system is intended to support are available;

d) Whether there are procedures available to support taking the action they believe is appropriate - if so they may choose to use these as guidance.

\subsubsection{A Case Where Operators Needed to Determine Whether Plant Behavior was the Result of Known Manual Actions or a Plant Fault}

In ISLOCA 1 a case arose that provided a specific example of a situation where operators need to engage in situation assessment in order to determine how to respond to a particular procedure step. The case occurred at a step in the LOCA procedure that asks whether pressure in all steam generators is "stable or increasing." If SG pressures are not all stable or increasing, the step directs the crews to return to Step 1 of the procedure (see Figure 3.1).

The purpose of this step is to check for the possibility of a faulted SG, which would cause SG pressures to decrease. In fact, there was no faulted SG, but there was a cooldown in progress, which also results in steam generator pressures decreasing.

Based on training and EOP background documents, crews are instructed that if they are taking actions that are producing a cooldown they should consider a decrease in SG pressure to be "stable or increasing." Thus, this is a case where response to a procedure step is based on situation assessment rather than literal interpretation of the procedure step. In determining how to respond to this procedure step the operators need to understand the intent behind the procedure step (i.e., its purpose is to check for the possibility of a faulted steam generator), determine whether the plant behavior they are observing can be explained by known influences on the plant (i.e., their own actions or those of automatic systems) or whether there nuay be a faulted SG, and decide how to respond to the procedure step based on that situation assessment.

27 The EDO or Emergency Duty Officer is a manager at the plant who wears a beeper while on call. He is notified and consulted in cases of abnormal plant conditions. 


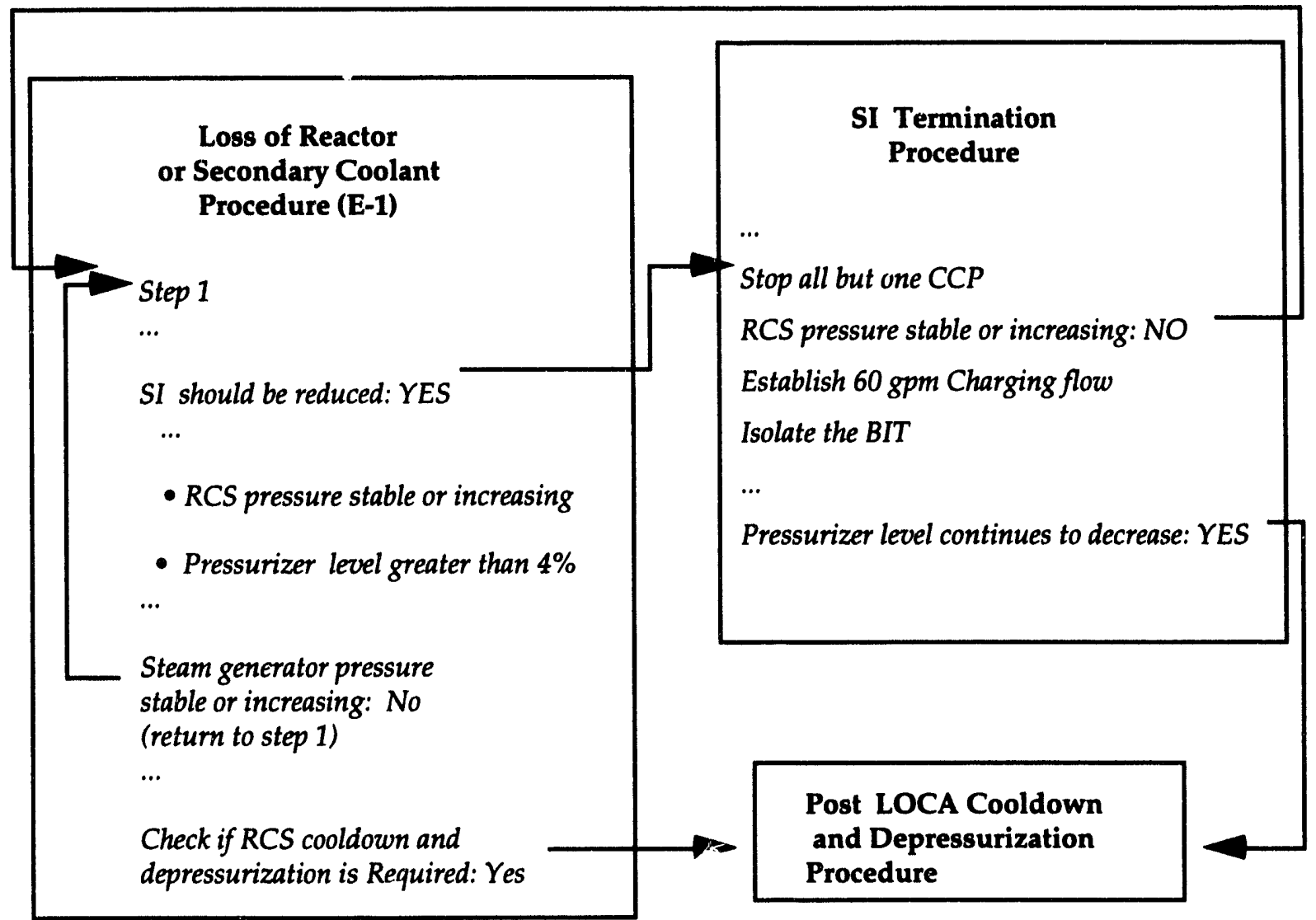

Figure 3.1 EOP transitions between LOCA procedure (E-1) and SI Termination Procedure.

Response Planning: Identifying goals and intent behind procedure steps

Six of the 11 crews were in the situation where steam generator pressures were decreasing when they got to this step. Five of the six crews decided to consider the steam generator pressure behavior "stable or increasing" and go forward in the procedure rather than return to step 1 as would be required from a literal reading of the procedure step.

The interaction of one of the crews (Crew G) when they came to the steam generator pressure "stable or increasing" step provides a clear illustration of the reasoning involved in determining how to respond to this procedure step. Below we provide an excerpt from their protocol. 


\section{Example of Crew Protocol Showing Role of Situation Assessment}

\section{Crew G}

\section{7:48:20 step 10}

Pressure in all SG -- BOP: Right now on a decreasing trend all of them.

7:49:38 SO (to SS): OK, We've got a decision to make here on this step here -- pressure in all generators stable or increasing, if it is no, we are going to be in this do loop; if we can say yes to this we can go on, stay in E-1 and we'll cooldown and depressurize, and that's what I think we need to be doing.

7:49:55 BOP: Right now they are decreasing.

SS: The definition of stable, are we controlling the decrease, you are feeding three of them at maximum rate.

7:50:09 SO: Not only that but RCS temperature is also decreasing from the SI flow.

SO: So I think the decrease in the steam generator pressure at this point is due to our feeding the generators, and the cooldown of the RCS.

7:50:32 SS: It's pretty much controlled, so it's stable.

SO: We have no indication of a faulted steam generator, that is the point.

7:50:45 SS: I would call that stable based on the parameters, since you are injecting 200,000 into it.

7:50:56 SO: How about RO and BOP, do you guys agree with that?

Both say OK.

This protocol excerpt illustrates several points. First, to determine how to respond to this step, the operators must actively consider the various known factors that are influencing steam generator pressure

NUREG/CR-6208 (e.g., RCS temperature due to SI; the fact that the steam generators are being fed at the maximum rate) and determine whether these are sufficient to account for the observed steam generator pressure behavior. In effect the step requires that they discriminate between a faulted steam generator and a decrease in steam generator pressure due to a cooldown.

A second point illustrated by this protocol is that the operators need to understand the intent of the procedure step to know what evidence to seek and consider in deciding how to respond. The fact that the crew understood the intent of the procedure step is illustrated by the statement made by the SO that "we have no indication of a faulted steam generator at this point." This suggests that they knew the intent of the procedure step, and evaluated the possibility of a faulted SG before responding. 28

Finally, the crew dialogue provides an example of a crew identifying a response goal based on the crew's situation assessment, and evaluating the procedure path with respect to achievement of this goal. This is illustrated by the early statement made by the $\mathrm{SO}$ to the SS: "If it is no we are going to be in this do loop; if we can say yes to this we can go on, stay in E-1 and we'll cooldown and depressurize, and that's what I think we need to be doing." By this statement the SO reveals that he thinks they need to be moving toward a cooldown and depressurization, and that answering "yes" to the steam generator pressure "stable or increasing" question will allow them to get to that point in the procedure more directly.

\subsubsection{Illustrative Protocol of Crew Performance in ISLOCA 1}

While the analysis above provides an overview of the performance of all the crews, and quantitative evidence of crews engaging in situation assessment and response planning, the analysis does not fully capture the extent of cognitive activity and crew dynamics observed in the scenarios. In this section we trace the performance of a crew that exhibited good situation assessment and response planning, to illustrate the types of situations that can arise in emergency events that require cognitive activity, and

\footnotetext{
$28 \mathrm{~A}$ faulted steam generator is equivalent to a steam line break.
} 
provide an example of good crew responses to those situations.

Below we present select portions of the protocol for Crew J. This crew did not see the RHR discharge pressure high alarm at the beginning of the event. Further, they were not provided the clue of water spilling outside the RHR pump room. As a consequence, they had to identify the RHR problem with only the PRT symptoms as a clue. This crew illustrates the use of schematic prints and alarm printouts to identify the leak into the RHR and attempt to isolate it. It also demonstrates the role of multiple crew members in generating the situation assessment. All crew members participated in the situation assessment and response planning. The $\mathrm{SO}$ explicitly solicits crew opinion and seeks consensus before taking actions not explicitly called out in the procedures.

Text in italics shows our annotations of the protocol that provide interpretation and comments on crew performance. The symbol '...' indicates that large segments of the crew dialogue have been omitted.

\section{Illustration of Crew Performance in ISLOCA 1 Crew J}

*** In this case the crew missed the RHR discharge pressure high alarm initially so they have no direct evidence that the leak from the RCS is going into the RHR. The instructor never provided them the clue that the leak in the Auxiliary Building was outside the RHR pump room. As a result the only clue they had of a problem in the RHR was the problem in the PRT. ${ }^{* * *}$ $\ldots$ 11:12 Reactor Trip.

...

*** While still in the entry procedure $(E-0)$, the crew showed evidence of beginning to think about possible sources of the leak into the PRT, although they took no action.***

11:15:30 BOP: It looks to me like our source of leakage is not the safeties or PORVs; however, it does look like it is going to the PRT, cause the PRT is over 200 degrees, so I think it is one of the other sources.

\section{1:15:50 Some other relief valve?}

11:15:55 BOP: Yea, I'm thinking it might be this relief valve right here on the seal water return

11:16:00 SO: OK, let me go through the immediate action steps, we'll let SS make his announcement and then we will pursue the leakage.

*** When they get radiation symptoms in containment and in the Auxiliary Building the SO shows evidence of considering the possibility of a link between the two symptoms . ***

11:20:55 SO: STA where do we have area rad high alarm? Can you determine that? Now we have a high high alarm.

11:21:00 STA: Sure, we have them all over containment, we have them all over the Auxiliary Building -- so it's in both places.

11:21:14 SO: Two pieces of information(announces to group) . We've got area rad hi and area rad high high, and we've got high area rad monitors all over containment, and all over the Auxiliary Building, so when we go looking for this leak that is something to keep in mind.

...

11:28:21 step 24 containment radiation is abnormal -so transitioning to E-1 loss of reactor and secondary coolant.

*** They had to actively attempt to identify the source of the leak into the Auxiliary Building when they got to step 12 in E-1 - at that point they show evidence of trying to pursue the source of the leak into the PRT. ${ }^{* * *}$

11:40:41 step 12 B -- try to identify and isolate the leakage.

$\cdots$

11:41:12 SO: Let's have a conference here for just a minute. Look at where we are at and where we are going. We are down to the point where we need to evaluate plant status. ... We've got abnormal containment and Auxiliary Building radiation, which tells us that we need to verify and isolate the leakage until we isolate the leakage. We need to try to determine where we need to go to isolate the leakage.

11:41:55 SO: The one thing, let me finish two points, the one thing that came in, that is weighing heavy on my mind, is that the first alarm that came in is PRT 
temp. high, level high, something like that, and then we started losing RCS levels, so what I'm thinking, like RO pointed out initially, whatever this leak is it is going into the PRT. We need to identify that source of leakage. Comments?

11:43:16 *** At this point the crew brings out prints and everyone is looking at them and participating in the discussion. They consider all the possible sources of leak into the Auxiliary Building. At first they dismiss the RHR relief valve possibility as implausible and consider the possibility of a reactor coolant pump seal leak off. When the leak continues after they isolate the reactor coolant pump seal leak off, they go to the alarm prints for a possible clue. It is at that point that they discover the RHR discharge pressure high alarm, which leads them to identify the problem in the RHR system. ***

BOP: We know we're pumping RCS through the Auxiliary Building right?

11:43:18 BOP: The only thing that we haven't isolated that could be coming from the RCS, we didn't get a temperature rise on the PORVs and all that stuff initially. At the same time we got the temp. rise here, I think, and I've thought all along, is that we've got leakoff here from reactor coolant pump seal leakoffs. OK, I don't have a good indication of that back there that I can tell, but if we isolated reactor coolant pump seal leakoffs we would know in a matter of a few seconds.

Why? Do you think that RCS pressure would start turning? Yea.

Letdown is isolated, RHR pump suctions those were de-energized and shut so it shouldn't be that.

Excess letdown was isolated when we started. We had no indication that it opened.

The only thing left is the pump seal leakoffs.

*** The portion below illustrates taking an action specifically intended to test a hypothesis as to the source of the ISLOCA ***

This one is not isolated still. Why don't we go ahead and do that?

SO: SS Do you agree?
SS: Where does that come in on the print?

Comes right in through those four valves here.

SS: I think that's a good idea. It's a suspected source; just go ahead and isolate it and see what happens.

*** The next portion illustrates that the crew carefully considers the potential for negative consequences before taking a control action that is not explicitly called for in the procedure. It also illustrates the practice of soliciting opinion and attempting to get consensus before taking an action that is not explicitly called for in the procedure. ***

11:46:25 SO: One question, what's the consequence of closing it?

BOP: We don't have reactor coolant pumps running.

So then where is the seal injection going?

It will go directly into the RCS.

It should not hurt anything.

SO: Yes, close the leak off isolation valves.

*** When the leak continues after they have isolated the pump seal leakoffs, they reconsider the possibility of a leak into the RHR .**

11:47:42 SO: Why did we rule out the RHR suction reliefs?

BOP: I'm not saying that should be ruled out. I'm just saying its not as likely as something that is energized. ...

11:48:11 BOP: What is the (RCS) pressure right now?

805. Still decreasing.

11:48:24 So that wasn't it apparently.

*** When their first hypothesis proves incorrect they then search for another possible explanation. This leads them to consider bringing out the alarm printout .***

$R O$ and BOP are in back discussing the source of the leak; SS and SO look at the prints. .*** ...

11:49:59 BOP: There are other inputs to the PRT. Is there anything else we can isolate? 
${ }^{* * *}$ All go to the prints - SS, RO, BOP, STA, SO ***

11:50:32 STA: So you are in the PRT prints, looking for the inputs.

11:50:35 SS: Basically, we have hit everything that makes sense; it doesn't mean that there is not something else here, but excess letdown has been isolated; RHR suctions de-energized, there is nothing we can do with those; this was just shut; normal letdown is isolated ...

11:51:05 SO: STA, what's the alarm printer show that came in first, before the trip?

STA: The alarm printer shows, PRT temp., PRT pressure, then it had pressurizer heaters on.

11:51:35 SO: There were two enunciators that came in at the same time and then there was a third one that came in that was over here somewhere.

STA: The one that came off of the printer, RHR alpha discharge pressure high.

11:52:16 SO: Gentlemen, let's re-group here for just a minute. I've got some information off the Alarm printer. The alarm printer says that the first alarm that came in prior to the event was RHR alpha discharge pressure high. That tells me, that would explain why we had the rad problems in the Auxiliary Building, and the problems in containment. It is obviously because we blew the rupture disk. I'm not sure I understand how we did that, how we ended up with PRT problems.

*** At this point the BOP suggests the hypothesis of backflow through leaking check valves. ${ }^{* * *}$

11:52:50 BOP: Leaking back through the RHR pump and then blew down to the PRT through the suction relief.

*** They consult prints.***

${ }^{* * *}$ Here again is an illustration of the SO attempting to keep the crew aware of his thinking and to seek consensus for proposed actions. This segment also illustrates again the fact that this crew makes sure there are no negative consequences of contemplated actions before the actions are taken. ${ }^{* * *}$
11:53:40 SO: I have some information for you. The relief valve is right here (to $R O$ and $B O P$ who are at the board).

11:53:45 RO: Here is a postulated solution. Here, the check valves are leaking; RCS leaks back through the RHR system pressurizes it, lifts the relief right here. The only way you are going to stop that is to take the RHR system out of service by isolating the discharge.

That's not something you normally do. You are disabling an ECCS system with a LOCA here. ...

We don't need the RHR pump right now because we are above the shutoff head.

Now is the time to do it.

SO: Let me get concurrence.

11:54:29 SO (to SS): Alright we have one option available to us that we need to evaluate.

SS: This is the relief that is lifting on us? These are normally closed down. What kind of pressure do you have in the RHR system?

$\cdots$

11:55:50 SO (to SS): We can isolate this pump, turn it off, shut these other -8809 alpha, we are on the alpha pump, isolate these check valves, and try to see if that is our source of leakage coming back this way and going out that relief valve.

11:56:14 BOP: As far as the suction valves, if the suction valves are leaking by, we are just screwed.

STA: So you are going to shut 8809 alpha off the alpha RHR pump and shut the 8701 alpha going to the loop hot leg, that's what we are trying to do.

It should be shut.

STA: Yea it should be. ...

RO: I think now is the time to do it before we are below the shutoff head of the RHR pump. Because we don't need it right now, we can shut it and see what happens. 
11:56:53 SS: Let me get a hold of the EDO, tell him what we propose to do.

...

11:59:32 **** Terminate the event .***

The protocol illustrates several important points about crew performance on the ISLOCA. First, it provides a clear case where identifying and isolating the ISLOCA required active situation assessment and response planning on the part of the crew. Initially, the only clue pointing to the RHR available to the crew was the PRT behavior. To correctly identify the source of the ISLOCA the crew had to actively consider the sources of input into the PRT. This led them to consider the possibility of an RHR problem. Similarly, active cognitive activity on the part of the crew was required to identify actions that could potentially isolate the leak.

Second, the protocol provides clear evidence of the use of additional resources to support situation assessment and response planning. The crew used the schematic prints to identify inputs into the PRT, and to identify which valves to close in an attempt to isolate the leak. In addition, the protocol illustrates the use of the alarm printout to identify symptoms that were previously missed. By the time the crew began to suspect an RHR problem, RHR discharge pressure was no longer high. The alarm printout provided the only remaining record of symptoms in the RHR.

Third, the protocol illustrates that the crew carefully checked for potential negative consequences before taking an action that was not explicitly called for in the EOP.

Fourth, the protocol illustrates the contribution of multiple crew members to situation assessment and response planning. The $\mathrm{SO}$ in this crew provided a particularly good example of "openness" in crew interaction. He solicited opinion, and sought consensus before taking actions not explicitly called for in the EOP.

\subsubsection{Variability in Crew Performance}

While the majority of the crews successfully identified the leak into the RHR and took action in an attempt to isolate the leak, variability in performance was observed. Variability was observed in identification of plant symptoms (e.g., the RHR alarm, the PRT rupture); identification of a problem in the RHR; and decisions with regard to what actions to take in an attempt to isolate the leak.

The crews varied in the extent to which they pursued symptoms and attempted to formulate a coherent explanation to account for all the symptoms observed. The contrast in performance of Crews $J$ and $L$ provide a case in point. For both crews the only symptoms they had pointing to the RHR problem were the abnormal symptoms in the PRT. One of the crews (Crew J) was able to identify the problem in the RHR by pursuing sources of input into the PRT, and then checking the alarm printout. The other crew never identified the problem in the RHR. As a result, they failed to take action that might have terminated the leak.

The ability to identify the RHR problem was important because it led the crews to identify actions that could potentially terminate the leak. The majority of crews correctly recognized that the leak into the RHR could be through the RHR isolation valves or through the check valves. The crews varied with respect to the extent to which they pursued those possibilities. While seven of the 11 crews considered the possibility of a leak through the RHR isolation valves only two crews called to have the valves reenergized. In the simulated event we ran, reenergizing the valves made no difference because the valves were closed. Had one of the valves been open, re-energizing them would have enabled the crew to detect the open valve and terminate the leak by closing it. 


\subsection{ISLOCA 2: ISLOCA Into RHR Leading to a Break in the RHR Heat Exchanger to the CCW}

In ISLOCA 2 we identified three cases where operators had to engage in situation assessment and response planning in order to deal with the situation.

The first case is the situation around which the ISLOCA 2 scenario was designed: a case where the procedure containing relevant guidance could not be reached within the EOP transition network. The analysis focused on the extra-procedural activities the crews engaged in in order to identify and isolate the ISLOCA into the RHR and the leak into the CCW.

The second case where a need for situation assessment was identified was a case that also arose in ISLOCA 1. There was a step in the LOCA procedure that asked whether pressure in all the steam generators was stable or increasing. Operators are instructed that if they are in a controlled cooldown they should consider a decrease in steam generator pressure to be "stable or increasing." This is a case where operators need to determine whether the plant behavior is the result of known manual and/or automatic actions or the result of a plant fault. As in ISLOCA 1 the analysis focused on how crews responded in cases where steam generator pressures were decreasing when they got to this step.

The third case was a situation where operators had to evaluate the appropriateness of a procedure path and take action to redirect the procedure path. In the case of two of the crews that ran in ISLOCA 2, a situation arose where the EOPs had crews repeatedly loop between the LOCA procedure and the SI termination procedure. The crews recognized that they needed to get out of this loop and get on to the Post-LOCA Cooldown procedure, but there was no proceduredriven way to do so. The analysis focused on how the crews evaluated the appropriateness of the procedure path, and what actions they took to redirect the procedure path.

\subsubsection{Characteristics of Participating Crews}

Eleven Crews from Plant 2 participated in ISLOCA 2. Two crews were eliminated from the data analysis because three or more crew members were aware of the event. Of the remaining nine crews, six were currently on shift, and three were composed of administrative staff. Crew size ranged from four to five people. In one case (Crew 1), two of the crew members (SS and BOP) had prior knowledge of the event. Those individuals did not actively participate in situation assessment and response planning.

\subsubsection{A Case Where the Procedure Containing Relevant Guidance Could not be Reached Within the EOP Transition Network}

In ISLOCA 2 there were two leaks that the operators needed to identify and attempt to isolate: the leak into the RHR from RCS, and the leak from the RHR heat exchanger into the CCW system.

In contrast to the EOPs of Plant 1, the EOPs of Plant 2 did contain a transition from the LOCA procedure to the ISLOCA procedure. A transition can be made based on radiation in the Auxiliary Building. So the scenario was timed so that radiation in the Auxiliary Building would not appear until after the crew had passed the transition step. Therefore, the crews in Plant 2 also had no direct access to procedural guidance for identifying and isolating the ISLOCA into the RHR and had to resort to extra-procedural activities to identify and isolate the leak.

Because of the dynamics of the event, only one of the nine crews observed in this event (Crew 4) met the procedural criteria to transition to the ISLOCA procedure from the LOCA procedure. The other eight crews had no procedural means of reaching the ISLOCA procedure.

In the case of six of the crews the criteria to transition to the ISLOCA were not met when they reached the transition step in the LOCA procedure. Specifically, in the case of these six crews, the CCW surge tank had not yet overfilled by the time the crews got to the step in the LOCA procedure asking about radiation in 
the Auxiliary Building. As a result there was no radiation in the Auxiliary Building when the crews got to that step; so the literal criteria for transitioning to the ISLOCA procedure were not met. ${ }^{29}$ Later, the CCW Surge Tank did overfill, spilling radioactive fluid onto the floor of the Auxiliary Building. At that point the criteria for transitioning to the ISLOCA procedure were met but by then the crews had passed the relevant step in the LOCA procedure. The EOP rules of usage provide no basis for returning to that step.

In the case of the remaining two crews they had no procedurally directed means of reaching the ISLOCA procedure because they never reached th $\div$ transition step. These crews transferred to the SI Termination procedure from the LOCA procedure before they got to the step that asked about radiation in the Auxiliary Building. From the SI Termination procedure they transferred directly to the Post-LOCA Cooldown and Depressurization procedure. As a result these two crews never reached a step that enabled a transition to the ISLOCA procedure. (See Figure 3.1 for an overview of the procedure transitions.)

The performance of these eight crews provided the opportunity to examine the role of situation assessment and response planning in guiding operator performance in a case where the procedure containing relevant guidance could not be reached within the EOP transition network.

While the ISLOCA procedure contained guidance on isolating the leak into the RHR, there was no procedure in the EOPs that explicitly addressed the leak from the RHR into the CCW. In order to identify and isolate the leak into the $\mathrm{CCW}$ the operators had to either diagnose the source of the leak on their own, or access the Off-Normal Procedure (OFN) for CCW System Malfunction as guidance. This provided an additional opportunity to examine how operators identify and isolate a leak in a case where no explicit procedural guidance was available.

The analysis focused on the extra-procedural activities the crews engaged in to identify and isolate the leak

${ }^{29}$ The simulated scenario was intended to be timed so that Auxiliary Building radiation symptoms did not appear until after the crews passed the step in E-1 that checks for Auxiliary Building symptoms. This was the case for all but one of the crews. into the RHR and the leak into the CCW. Specifically, we examined:

- Whether crews identified a problem in the RHR system, and if so on what basis;

- Whether crews recognized that the symptoms across systems (i.e., symptoms in containment, symptoms in the RHR, and symptoms in the $\mathrm{CCW}$ ) all resulted from the same underlying fault, and if so on what basis;

- The actions that crews took to identify and isolate the ISLOCA, given that they could not reach the relevant procedure within the EOP transition network;

- The actions that crews took to identify and isolate the source of the leak into the $\mathrm{CCW}$, given that the only procedure advice available was in an OFN.

Situation Assessment: Recognizing that it was not a simple LOCA by the absence of expected symptoms or presence of unexpected symptoms.

In the case of ISLOCA 2 the RHR discharge pressure high alarm was suppressed. As a result the primary indicator of a problem in the RHR was missing. The remaining indicators of a problem in the RHR were indirect.

The first alarms that came in, low pressurizer pressure and level, suggested a LOCA inside containment. The primary indicators that this was not a simple LOCA inside containment were (1) abnormal activity in the PRT and its eventual rupture and (2) the fact that containment symptoms that would be expected in the case of a LOCA inside containment (i.e., increases in humidity and radiation inside containment) were not present. We examined whether the crews noticed these unexpected plant behaviors and whether that led them to identify a problem in the RHR.

Seven of the nine crews noticed the PRT rupture early in the event. One crew (Crew 7) did not mention noticing the PRT rupture until 40 minutes after the reactor trip. One crew (Crew 3 ) never verbally communicated the PRT rupture. In the case of this latter crew, the SS indicated that he had noticed the 
PRT rupturing but he never mentioned it to the rest of the crew. As a result at no point in the event did the SO realize that the PRT ruptured.

Five of the seven crews who noticed the PRT rupture early checked for possible sources of input into the PRT. This is an example of knowledge-driven monitoring in search of an explanation for an unexpected plant behavior. As will be shown, the search for sources of input into the PRT led these crews to identify RHR symptoms that they otherwise might not have noticed.

Table 3.3 presents the point at which crews recognized that the event was not a simple LOCA inside containment, who mentioned it, the reason given for that conclusion, and the action taken as a result. Five of the crews recognized early that the event was not a simple LOCA because of the absence of symptoms they expected based on their situation assessment. These crews indicated that given the rate of level and pressure drop in the pressurizer, they expected to observe more symptoms in containment (increasing pressure and humidity) than they saw. All five of these crews concluded that the event was not a simple LOCA before they got any positive evidence of a problem outside containment.
The remaining four crews recognized that the event was not a simple LOCA based on observation of plant symptoms that were unexpected given a LOCA hypothesis. In three cases this was based on observation of symptoms in the PRT. In the last case (Crew 10) it was based on observation of RHR symptoms, which in turn were found in the process of searching for an explanation of the symptoms in the PRT.

Identification of unexpected plant behavior led the crews to search for an explanation. One crew searched for a potential steam generator problem. One crew called the Auxiliary Building operator to search for a potential leak. One crew specifically suspected a leak from the RCS to the RHR via the isolation valves on the suction side of the RHR and called to have them re-energized. These actions anticipated later EOP steps.

These results provide specific instances where expectations guided operator performance. Recognizing that the event was not a simple LOCA allowed the operators to realize that there was an additional problem that needed to be identified and solved.

Table 3.3 ISLOCA 2. Crew recognition that event was not a simple LOCA. ${ }^{30}$

\begin{tabular}{cccc}
\hline Crew No. & Crew Member & Reason Given & Action \\
\hline 1 & ct & not enough containment symptoms & none \\
3 & RO & not enough containment symptoms & none \\
4 & SS & not enough containment symptoms & none \\
6 & SO & not enough containment symptoms & watch for SG problem \\
7 & SO & not enough containment symptoms & calls to search Aux. Building \\
8 & SS & PRT symptoms & check sources \\
9 & SS & PRT symptoms & re-energize isolation valves \\
10 & BOP & RHR symptoms & none \\
11 & ct & PRT symptoms & check sources \\
\hline
\end{tabular}

30 'ct' ="cannot tell.." It means that the information could not be determined from the videotape. 


\section{Situation Assessment: Hypothesizing the cause of the RHR symptoms}

Since the RHR discharge pressure high alarm was suppressed there were no salient cues of a problem in the RHR. There were symptoms of an RHR problem available in the control room; specifically, there were RHR discharge pressure and temperature meters that read abnormally high and vacillated in value as the RHR relief valve opened and closed. However, the operators had no alarms or procedure steps to direct them to check those meters. Nevertheless, all of the crews eventually detected the RHR symptoms, and so identified a problem in the RHR.

One of the most striking characteristics of performance in ISLOCA 2 is the degree to which the crews pursued potential sources of leaks into the PRT. This search allowed them to identify abnormal RHR symptoms that they would otherwise not have observed. Seven of the crews detected the RHR symptoms early, as a result of checking for potential sources of input into the PRT.

One of the crews during the debriefing provided an articulate description of the situation assessment and knowledge-driven monitoring activities they engaged in during the scenario. The crew indicated that they were aware of all the sources of input to the PRT and considered each in turn. One crew member said "Given the amount we were losing it just appeared to be the RHR, it's the largest relief valve. We didn't have monitoring for it, we had monitoring for the others and they were fine." When they got the CCW alarm they first thought to check the RCP thermal barriers. They thought of the RHR in the context of what could be getting into the PRT. That led them to detect that RHR discharge pressure was high and temperature was very high. This led them to search for ways that fluid in the RHR could get to the CCW. They identified the RHR heat exchanger as a possibility because it is the biggest interface between the two systems. During this period they referred to schematic prints for inputs to the PRT and interfaces between the RHR and the CCW systems.

Not all crews noticed the RHR symptoms in the search of an explanation for the symptoms in the PRT. Crew 3, which had not detected the PRT symptoms, noticed the RHR symptoms incidentally as they were performing a later step in the EOP. One of the steps in the Post-LOCA Cooldown and Depressurization procedure had the crew stop the Bravo SI pump. In taking this action the BOP noticed the Bravo RHR discharge pressure was not behaving the way he expected. He expected to see Bravo RHR pumv discharge pressure to go down. Instead it stayed at around $400 \mathrm{lb}$. This violated expectation led him to suspect a problem in the RHR. This aspect provides another illustration of the power of failed expectations in guiding situation assessment.

Table 3.4 shows what explanations the crews generated for the RHR symptoms. As can be seen, the failed isolation valves between the RCS hot leg and the suction side of the RHR system were considered at least as often as the failed check valve hypothesis.

Table 3.4 ISLOCA 2. Hypothesized explanation for RHR problem.

\begin{tabular}{cccc}
\hline Crew No. & First Hypothesis & Crew Member & Revised Hypothesis \\
& & & $\mathrm{ct}$ \\
\hline 1 & isolation valves & $\mathrm{ct}$ & $\mathrm{n} / \mathrm{a}$ \\
3 & check valves & $\mathrm{SO}$ & isolation valves \\
4 & check valves & $\mathrm{SS}$ & isolation or check valves \\
6 & isolation valves & BOP & isolation valves \\
7 & check valves & SS & ct \\
8 & ct & $\mathrm{n} / \mathrm{a}$ & isolation or check valves \\
9 & isolation valves & SS & isolation or check valves \\
10 & check valves & BOP & isolation or check valves \\
11 & isolation valves & $\mathrm{ct}$ &
\end{tabular}


Situation Assessment: Recognizing that symptoms in the $C C W, P R T$ and Containment had a common source

One of the questions we examined was whether crews recognized that the symptoms across systems all resulted from the same underlying fault.

One aspect of this question is whether the crews recognized that the containment symptoms were caused by the rupture of the PRT. Five of the seven crews who noticed the PRT rupture early explicitly connected the containment symptoms to the rupture of the PRT, thus illustrating the role of situation assessment in explaining observed symptoms.

Another aspect is whether crews recognized that the PRT symptoms were caused by the problem in the RHR. All eight crews who had observed the PRT rupture correctly attributed the PRT symptoms to the release of fluid from the RHR via the relief valve to the PRT.

A third aspect is whether crews recognized that the symptoms in the CCW were caused by the problem in the RHR. All the crews identified a problem in the $\mathrm{CCW}$ when alarms indicated high radiation in the $\mathrm{CCW}$. Of the nine crews, seven eventually recognized that the problem in the $\mathrm{CCW}$ was due to a leak from the RHR system.
Situation Assessment: Explaining symptoms in the CCW system

Table 3.5a presents the first hypothesis the crews generated to explain the symptoms in the CCW system, who generated it, and the reason given for that explanation. The first column indicates whether the crews identified a problem in the RHR system before the CCW alarm came in. As can be seen there was a difference in the hypotheses generated between the crews who had detected a problem in the RHR before the CCW alarm came in, and those who detected the CCW alarm first. The three crews who were not aware of a problem in the RHR first suspected a leak into the $\mathrm{CCW}$ from the service loop. In contrast, three of the six crews who already knew there was a problem in the RHR, suspected a leak into the $\mathrm{CCW}$ from the RHR.

Table 3.5b shows whether that initial hypothesis was revised later, and if so, what the revised hypothesis was. Of the three crews who detected the CCW alarm first, two later revised their explanation of the cause of the problem in the CCW when they identified a problem in the RHR system. By the end of the event five of the nine crews correctly identified that the leak into the CCW came from the RHR heat exchanger.

Table 3.5a ISLOCA 2. First hypothesis generated to explain the CCW problem. ${ }^{31}$

\begin{tabular}{ccccc}
\hline Crew No. & $\begin{array}{c}\text { Noticed RHR Problem } \\
\text { Before CCW Problem }\end{array}$ & First Hypothesis & Crew Member & Reason Given \\
\hline 1 & yes & service loop & ct & ct \\
3 & no & RCP thermal barriers & SO & CCW alarm \\
4 & yes & service loop & SO & symptom after swap CCW train \\
6 & no & service loop & SO & CCW alarm \\
7 & no & service loop & SS & symptom after swap CCW train \\
8 & yes & ct & n/a & n/a \\
9 & yes & RHR pump seal cooler & STA & RHR problem \\
10 & yes & RHR pump seal cooler & ct & RHR problem \\
11 & yes & RHR pump seal cooler & STA & RHR problem \\
\hline
\end{tabular}

\footnotetext{
${ }^{31}$ The OFN procedure has the crews transfer the service loop to the other CCW train. When the crews swap the CCW train it causes radiation alarms in the second train. This led some crews to incorrectly conclude that the leak was from the service loop.
} 
Table 3.5b ISLOCA 2. Revised hypothesis to explain the CCW problem.

\begin{tabular}{cccc}
\hline Crew No. & Revised Hypothesis & Crew Member & Reason Given \\
\hline 1 & RHR heat exchanger. & ct & ct \\
3 & ct & $\mathrm{n} / \mathrm{a}$ & $\mathrm{n} / \mathrm{a}$ \\
4 & no & $\mathrm{n} / \mathrm{a}$ & $\mathrm{n} / \mathrm{a}$ \\
6 & RHR heat exchanger & RO & RHR symptoms \\
7 & RHR pump seal cooler & SS & RHR symptoms \\
8 & RHR heat exchanger & SO & OFN logic \\
9 & RHR heat exchanger & SS & told by instructor \\
10 & RHR heat exchanger & ct & ct \\
11 & service loop & SO & symptom after swap CCW train \\
\hline
\end{tabular}

Response Planning: Employing additional resources to identify the source of the leaks

The previous analysis focused on situation assessment activities. We also examined the actions that the crews took in attempting to isolate the leaks into the RHR and the CCW system. The analysis focused on the additional resources they accessed to support them in identifying and isolating the leaks, the actions they took, and the reasons for selecting those actions.

At least three of the crews used the CCW OFN for guidance. In addition, four of the crews either transitioned to the ISLOCA procedure or used it as guidance. As mentioned earlier, only one crew (Crew 4) had radiation symptoms in the Auxiliary Building when they reached the step in the LOCA procedure that checked for Auxiliary Building radiation, which is the literal criterion for transferring to the ISLOCA procedure. The other three crews (Crews 3, 6, and 11) accessed the ISLOCA procedure for guidance based on their own assessment of the situation. Finally, at least six of the crews accessed schematic prints in attempting to identify and isolate the leaks in the $\mathrm{CCW}$ and the RHR systems.

\section{Response Planning: Attempting to isolate leaks into the RHR and the CCW}

One question was what actions did the crews decide to take in attempting to isolate the leak into the RHR and what guided the identification of that response action, given that relevant guidance on isolating an ISLOCA could not be accessed via the EOP transition network.

All nine crews considered the possibility of a leak through the isolation valves between the hot leg of the RCS and the suction side of the RHR pump and called the Auxiliary Building to have them re-energized. In all cases the decision to re-energize the isolation valves was based on their situation assessment rather than an explicit procedure step. In one case (Crew 4) the crew decided to wait for an explicit step in the ISLOCA procedure before performing this action.

Seven of the nine crews decided to isolate the RHR train. In two cases (Crews 4 and 6) the action was performed as part of the ISLOCA procedure. In the case of the other five crews the action was identified based on their situation assessment.

The second question was what actions did the crews decide to take in attempting to isolate the leak into the $\mathrm{CCW}$ and what guided the identification of that response action, given that the only procedural guidance available was in an OFN procedure. Specifically, we examined whether the crews considered isolating the RHR heat exchanger, which was the source of the leak into the CCW and the basis for identifying that response action.

All of the crews considered the RHR heat exchanger as a possible source of the leak. In three cases (Crews 
3,7 and 8) the possibility of isolating the RHR heat exchanger was based on the CCW OFN.

At least seven of the nine crews took action to isolate the RHR heat exchanger. One crew (Crew 3) decided against isolating the heat exchanger because the $\mathrm{SO}$ did not believe it could be the source of the leak.

\subsubsection{A Case Where Operators Needed to Determine Whether Plant Behavior was the Result of Known Manual Actions or a Plant Fault}

As in the case of ISLOCA 1, we examined how the crews responded to the step in the LOCA procedure that asked whether steam generator pressures were stable or increasing. In the case of six of the nine crews steam generator pressure was decreasing when they got to that step. Of those six crews, four decided to consider the steam generator pressure behavior 'stable.' This is similar to the behavior observed in ISLOCA 1.

This case exemplifies a situation where operators based their decision on their situation assessment in order to move expeditiously through the procedures. In particular, in this situation crews needed to discriminate energy effects (e.g., cooldown caused by known influences) from mass effects (e.g., a faulted steam generator) in order to know how to respond to the procedure step.

\subsubsection{Cases Where Operators Evaluated and Redirected the Procedure Path}

Response Planning: Identifying Goals and Evaluating the Procedure Path

Several of the protocols provided evidence that the crews in question were reasoning at two levels. They were engaging in situation assessment and goal identification. Simultaneously they were monitoring the procedures they were following to ensure that the actions specified in the steps were appropriate to the situation as they perceived it, and that the procedure path would result in achievement of plant goals.
In several instances the operators explicitly discussed whether the procedure path they were on would enable them to achieve important goals in a timely manner or whether they needed to take action to redirect themselves in the procedure network.

Below we present segments of two protocols where the crews provided evidence that they were monitoring the procedure path they were on. Both cases involve crews that transitioned from the LOCA procedure (E-1) to the SI Termination procedure. In both cases the crews recognized that given the size and nature of the leak, a high priority goal was to begin cooldown and depressurization. This step entails going to the Post-LOCA Cooldown and Depressurization Procedure. In both cases crew members raised the question of whether the procedure path would get them to the Post-LOCA Cooldown and Depressurization procedure in a timely manner. They actively engaged in problemsolving to identify a procedure path that would get them from where they were to the Post-LOCA Cooldown procedure.

Figure 3.1 (see page 29) provides a diagram of the EOP transitions among these three procedures. The Post-LOCA Cooldown and Depressurization procedure can be reached from either E-1 or the SI Termination procedure. As shown in Figure 3.1, in E1 there is a step that checks whether SI should be reduced. If the answer is yes the crews are transitioned to the SI Termination procedure. From there, if pressurizer level continues to decrease, the crews are transitioned to the Post-LOCA Cooldown and Depressurization procedure.

In one of the protocol segments we present the crew was transitioned to the SI Termination procedure. They recognized that they had a large enough leak that they needed to get to the Post-LOCA Cooldown procedure, but when they got to the step in the SI Termination procedure that checks for transition to the Post-LOCA Cooldown procedure, the literal criteria for transition were not met. The question they faced was how to proceed to the Post-LOCA Cooldown procedure, given that they did not meet the literal criteria for transition.

It is also possible to transition from the SI Termination procedure back to the LOCA procedure. In the SI Termination procedure there is a step that checks that RCS pressure is stable or increasing. If the answer is 
no the crews are transitioned back to step 1 in E-1. The second protocol segment we present is a case where the crew was repeatedly transitioned between E-1 and the SI Termination procedure. The question they faced was how to get out of this loop and move on to the Post-LOCA procedure.

\section{Case 1: Redirecting the procedure path}

In the first case we present the crew transitioned to SI Termination from the LOCA procedure (E-1) because RCS pressure was increasing and pressurizer level was greater than $4 \%$. Once in the SI Termination procedure, however, they wondered whether they were on an appropriate procedure path. They recognized that they needed to get to the Cooldown and Depressurization procedure and discussed among themselves which procedure path would get them there. They decided to stay in the SI Termination procedure with the intention of transitioning to the Post-LOCA procedure from there. However, when they got to the step in the SI Termination procedure that enabled a transition to the Post-LOCA procedure, they found that they did not meet the literal criteria for the transition. The SO decided to make a judgment call and transition to the Post-LOCA Cooldown procedure nevertheless.

\section{Evaluating and Redirecting the Procedure Path Crew 10}

This segment begins in the SI termination procedure. When they got to the step in the SI Termination procedure that asks about $R C S$ pressure, $R C S$ pressure was increasing so they did not transition back to E-1. A little later RCS pressure thined back and started to decrense. At this point the $R O$ asks the crew whether maybe they should have transitioned back to E-1 since they still have a LOCA in progress.

10:20:25 RO: One question gentlemen, I'd like to bring up maybe as a point. We just stepped by the step a little bit ago. Maybe we didn't wait long enough here for stable and increasing. We are in a loss of reactor coolant right now and would E-1 not be a good place to be? I mean if we are stabilizing right here with BIT flow going and everything else, we can pretty much just figure that we're losing a lot of volume still somewhere, probably to containment, so isolating the
BIT is just going to get us back to re-establish the BIT. 32

The SO acknowledges the RO's point, but argues that they are on a correct procedure path. He indicates that where they want to be is in the Post-LOCA Cooldown and Depressurization Procedure, and there is a procedure path that will get them to that procedure from where they are.

10:20:50 SO: OK, right, We were increasing after that point.

BOP: Just for a little while.

SO: Until we established the $60 \mathrm{gpm}$ charging. Now we'll get off of the BIT. We'll come over to see if we can stop SI. We're not going to be able to. We'll get into ES-11 POST-LOCA COOLDOWN AND DEPRESSURIZATION, and that's where we need to head right now, so that's what we are going to do.

10:21:22 BOP: I understand.

...

SO: Need to Stop the CCP then close the BIT inlet and BIT outlet valves.

RO: Pressurizer level is indeterminate right now. ...

They reach the procedure step in the SI termination procedure that allows them to transition to the Post-LOCA Cooldown and Depressurization procedure; however, pressurizer level is increasing slightly, so they do not meet the literal criteria for transitioning to the Post-LOCA Cooldown and Depressurization procedure, which is a decrease in pressurizer level. At this point the SO announces that he is making a judgment call and transitioning to the Post-LOCA procedure (ES-11) in spite of the fact that they do not meet the literal transition criteria.

10:23:00 SO: Take a minute here to evaluate everything gentlemen.

Pressurizer level is slowly indicating an increase.

Very slow decrease on RCS pressure.

I have a trend here on the computer of increasing level in the pressurizer.

${ }^{32}$ BIT refers to Boron Injection Tank. 
10:24:05 SO: I'm going to have to make a judgment call, we're going to ES-11... Doing it on max. charging isn't the way to be going. Re-align through the BIT and go to ES-11.

This protocol segment illustrates several points. First, it illustrates openness in interaction among crew members. In this case the RO raised a concern with regard to appropriateness of procedure path. Second, it illustrates evaluation of the current procedure path in light of the crew's perception of appropriate goals, and redirection of the procedure path is judged appropriate. When the RO raised his concern, the SO affirmed that there was a procedure path that would allow them to get from the SI Termination procedure to the Post-LOCA Cooldown procedure. Later, when it looked like they might miss the transition to the Post-LOCA Cooldown procedure because they did not explicitly meet the transition criteria, the SO decided to make a judgment call and transition to the Post-LOCA Cooldown procedure in any case.

\section{Case 2: Understanding the transition logic among EOPs}

The next protocol segment illustrates the importance of understanding the transition logic among EOP procedures. This crew gets in a procedure loop that keeps them from getting to the Cooldown and Depressurization procedure. The STA recognizes that a high priority goal is to get to the Cooldown and Depressurization procedure; he actively engages in problem solving to identify a way to get out of the procedure loop.

The step in the LOCA procedure intended to determine whether SI should be reduced asks whether pressurizer pressure is stable or increasing. In the case of this crew, pressurizer pressure was increasing slightly when they reached that step. This meets the criteria for SI termination so the crew transitioned to the SI Termination procedure. That procedure has them turn off all but one Centrifugal Charging Pump (CCP). Turning off the CCPs results in pressurizer pressure and level going down. As a result, when the crew gets to the step in the SI Termination procedure that asks whether pressurizer pressure is stable or increasing, pressurizer pressure is decreasing. As a result the crew is transitioned back to step 1 of the LOCA procedure. Because pressurizer level is less than $4 \%$ they turn the CCPs back on. When they again get to the SI termination criteria step in the LOCA procedure, pressurizer pressure is again increasing slightly, and the EOP has them transition to the SI termination procedure for the second time. Because the flow in from the CCPs just keeps up with the flow out from the leak, the crew could be kept in this loop between the LOCA and the SI termination procedure indefinitely. The STA recognizes this problem, and searches for a way to get out of the procedure loop and get to the Cooldown and Depressurization procedure. ${ }^{33}$

\section{Understanding the Transition Logic Among EOPs Crew 9}

9:57 *** This segment starts in the SI Termination Procedure to which they have been transitioned from the LOCA procedure (E-1) for the second time. ${ }^{* * *}$ $\ldots$

9:58 Stop alpha centrifugal charging pump. ...

SO: If we get less than $4 \%$ on the pressurizer, we'll start up the CCP again.

\footnotetext{
${ }^{33}$ In this case the STA suggestion for how to get out of the loop between the LOCA procedure (E-1) and the SI termination procedure is to not turn the CCPs back on so that pressurizer pressure will not be increasing when they get to the step in E-1 that directs a transition to the SI termination procedure based on increasing pressurizer pressure. An expert in the use of the Westinghouse ERG has suggested that a preferable resolution would have been to turn on the CCPs as directed by E-1, but then not make the transition to the SI termination procedure on the second pass even though the literal criteria for transition were met. The argument made is that once the crew transitions back to E-1 from the SI termination procedure, it has been determined and should be understood that the reason for the transition back to E-1 was a small LOCA that does not meet the SI termination criteria and therefore, the SI termination procedure should not be reentered. The crew would then eventually get to the step in E-1 that would transition them to the Post-LOCA Cooldown and Depressurization procedure. Note that this alternative resolution also depends on a) an accurate situation assessment; b) a deep understanding of the rationale behind procedure steps and procedure transition logic; and c) a deviation from the literal procedure step criteria for transition to the SI termination procedure.
} 
STA: Well, lets think about it. We could leave it off and then transition back to E-1 and get farther in E-1 this time. We're going to have to get cooldown anyway so we're never going to get there this way. There' s plenty of subcooling. I'd leave it off and transition back to E-1. That's a recommendation. You'll get farther in E-1 next time.

*** STA and SO look at EOPs together ***

STA to SO: You're not going to go back to step 1 (referring to a step in E-1 that would loop back to step 1 if $R C S$ pressure is increasing or SG pressure is decreasing). You're going to go on through here. This will get you to POST-LOCA COOLDOWN AND

DEPRESSURIZATION. That's where we want to be.

STA: I'd say leave the CCP off and let E-1 get you to ES-11 (the Post-LOCA procedure).

*** SO and SS review the LOCA and SI Termination procedures to figure out how to get out of the continuous loop - they eventually agree to do what the STA suggests.

***

This protocol segment provides a concrete example of a case where a crew understood the logic of the EOP transition network, actively monitored whether adequate progress was being made toward high priority goals, and when it was determined that the current procedure path was unproductive, actively engaged in problem-solving to identify a way to get on a more appropriate procedure path, while still staying within the EOP framework.

The discussion between the STA, the SO, and the SS reveals that they have an accurate situation assessment. They understand that the leak is just barely being compensated by the CCPs. They also understand that a primary goal given the situation is to get to cooldown and depressurization expeditiously. Finally, they understand the structure of procedure transitions among the LOCA procedure, the SI Termination procedure, and the Post-LOCA Cooldown and Depressurization procedure. When they realize that they are in a procedure loop that is keeping them from getting to the Post-LOCA Cooldown and Depressurization procedure, they actively engage in problem-solving to identify a way to get out of the loop. In this case, the STA comes up with the strategy that will allow them to get past a procedure step that is putting them in a loop. Specifically, he suggests that they not turn on the CCPs so that pressurizer pressure would be decreasing when they get to the procedure step that checks for SI termination criteria.

This protocol segment points out that cases can arise where crews need to engage in reasoning about the procedure logic, and how best to respond to procedure steps to get to the point in the procedure where they need to be. It is reasonable to assume that this case is not unique and that similar situations requiring active monitoring of the procedure path will arise in emergency events. This analysis suggests that it is important that crews reason at two levels. They need to engage in situation assessment and goal identification and they need to reason about the strategies underlying the EOPs, and the EOP transition network logic in order to assess whether the current procedure path they are on will enable them to achieve plant goals in a timely manner, or whether they need to take action to redirect themselves within the EOP network. The implications of this conclusion for training are discussed in Section 5.

\section{Other cases where crews evaluated and redirected the procedure path}

Other examples exist of crews monitoring procedure steps for appropriateness to the situation. In some cases a given procedure step was judged to be inappropriate given the particular state of the plant. For example, in one case a crew (Crew 6) reached a step in the LOCA procedure that said "Establish CCW flow to RCP thermal barriers." At the point the crew got to this step they knew they had RCS fluid leaking into the CCW but had not yet identified the source of the leak. The SO decided that given the problem in the CCW it wo. not be appropriate to establish CCW flow to the 'P thermal barriers and does not take this action. SL. I'm trying to think here. They want us to restore $\mathrm{CCW}$ to the reactor coolant pump thermal barriers as part of this step. I'm not sure it would be advisable at this time."

In another case the crew identified a situation where the actions they had taken to deal with one problem prevented them from accomplishing a step in the EOPs. In this case the crew (Crew 7) had divided into two subgroups with the $\mathrm{SO}$ and $\mathrm{RO}$ continuing with 
the EOP to get to cooldown and depressurization, and the SS and BOP using the CCW OFN to try and identify and isolate the leak into the CCW. At some point the SO reached a step in the Post-LOCA Cooldown and Depressurization procedure that asked to establish CCW flow to RCS at some target value. This was not possible, however, because the SS had isolated the CCW service loop as part of his attempts to identify and isolate the leak in the CCW. At this point the SS who had been closely coordinating with the SO says "We isolated that (the CCW service loop) when we started to encounter our CCW problem. I think we pretty well determined it is on the Bravo Safety loop. Why don't we go ahead and restore service loop alpha and get CCW back?"

This case provides an example of a situation where a crew needed to understand the effect of plant state on the ability to perform procedure steps, the goals to be accomplished by the procedure step, and how to achieve these goals given the current plant state. In this particular case, the crew had to determine that it was possible to place the CCW in service in spite of the leak in the CCW system, and to reconfigure the $\mathrm{CCW}$ system to allow this (i.e., switch to the A train CCW).

\section{Catching errors}

A final example of crews monitoring and evaluating the appropriateness of procedure steps, illustrates the role of situation assessment and response planning in catching errors. In this case a crew (Crew 4) caught a small error in the logic of a procedure step in the Reactor Trip or SI procedure. As part of a step to check for a steam generator rupture, one of the substeps read "High radiation from any Steam Generator steamline radiation monitor." According to the EOP two column format, if that criterion is not met, the operator is directed to the Response Not Obtained (RNO) column which says to go to the Steam Generator Tube Rupture procedure.

In this case the operator knew that having no steam generator steam line radiation monitor alarms was not an indicator of a steam generator tube rupture and that it would not be appropriate to transition to the Steam Generator Tube Rupture procedure. He consulted with the SS who concurred, and they decided not to transition to that procedure. The dialogue they engaged in is presented in the protocol segment below.

\section{Catching Errors \\ Crew 4}

13:30:31 SO: SS, I'm going to deviate slightly here from the approved method of using these procedures because if I answer this question correctly, I go to the RNO column which sends me to tube rupture.

13:30:45 SO: This step right here says high radiation from any SG steamline radiation monitor (Step $24 \mathrm{~d}$ ). The answer is no. That puts me over here. I don't want to go there, I want to go on.

SS: I understand, and approve.

The protocol segment above provides an example where the crew monitored the appropriateness of procedure steps based on their own situation assessment and goal identification. In this particular case the SO knew that there was no evidence of a tube rupture present and that it was inappropriate to transition to the steam generator tube rupture procedure. This allowed him to detect a small error in the procedure, and obtain concurrence from the SS to deviate from the literal statement of the procedure step. 34

\subsubsection{Variability in Crew Performance}

While most crews succeeded in identifying the source of leaks into the RHR and the CCW and in identifying the correct response while attempting to isolate the source of the leak, there was variability in performance across crews. Some crews identified the RHR problem sooner in the event than others. This enabled them to quickly recognize that the leak into the CCW was from the RHR, to localize the leak to the RHR heat exchanger, and to take action to isolate the RHR heat exchanger.

\footnotetext{
${ }^{34}$ The error in the wording of the EOP step has since been corrected.
} 
One crew (Crew 3) had particular difficulty in identifying and isolating the leaks. This crew exhibited poor communication, and an inability to engage in systematic diagnostic activity to identify and isolate the leaks. In particular, while at least one member of the crew noticed the PRT rupturing, that information was not communicated to the SO. As a result the crew did not identify a problem in the RHR until late in the event, and then only because the RO incidentally noticed abnormal RHR behavior while performing a procedure step. The crew accessed the CCW OFN procedure but failed to follow the procedure systematically in order to identify and isolate the leak. One reason was that the SO seemed to incorrectly equate the fact that they had been told that the Bravo RHR train was out of service with it being already isolated. As a result, the SO believed the RHR heat exchanger was already isolated. This confusion was never corrected by any other member of the group. As a result, the crew never attempted to isolate the Bravo RHR heat exchanger in spite of the fact that the CCW OFN explicitly includes a step to isolate the RHR heat exchanger.

\subsection{Loss of Heat Sink 1: Total Loss of Secondary Heat Sink (Feedwater Never Recovered)}

In the Loss of Heat Sink 1 scenario we identified three cases where operators had to engage in situation assessment and response planning to deal with the situation.

Two of these cases had been explicitly designed into the scenario. These were: (1) a case where operators needed to discriminate plant behavior that was the result of known factors (i.e., an operator induced cooldown) from plant behavior that signaled an additional plant fault and (2) a case where operators had to decide whether to manually initiate a safety system based on consideration and balancing of multiple goals related to safety.

The third case was an example of a situation where operators had to evaluate the appropriateness of a procedure path. We observed three cases where crews got to a step in the EOP that called for a transition to a different procedure, but failed to make the transition. Instead, they continued with the steps in the procedure they were following. In all three cases the crews recognized their error within two steps. The process by which the crews recognized the mistake they made and took action to get back on the right procedure path provided an example of how response plan monitoring allows operators to catch and correct their own errors.

\subsubsection{Characteristics of Participating Crews}

Ten crews performed the LHS 1 scenario. Of these, two were eliminated from the analysis because an inadvertent SI occurred during the RCS depressurization early in the event. Of the remaining eight crews four were staff crews and four were crews currently on shift. Crew size ranged from four to five individuals. Two of the staff crews (Crews B and D) included one individual who had prior knowledge of the event. These were training instructors, who filled the role of STA or SS. These individuals did not offer opinions or participate in situation assessments or response evaluations.

\subsubsection{A Case Where Operators Needed to Determine Whether Plant Behavior was the Result of Known Manual and/or Automatic Actions or the Result of a Plant Fault}

One of the questions we examined was the ability of the crews to identify the presence of a leak on the primary side (i.e., the leaking pressurizer PORV), given that the focus of the procedures and operator attention was on the loss of heat sink event on the secondary side of the plant.

To identify the leak the crews had to recognize that the behavior on the primary side could not be explained by the known factors influencing plant behavior. The early symptoms of the leaking pressurizer PORV, a decrease in pressurizer pressure and level, could be attributed to a cooldown resulting from the actions the operators were taking on the secondary side of the plant. Later symptoms (pressurizer level going up while pressure continued to go down, a bubble forming in the reactor vessel, and activity in the PRT) could not be accounted for 
by a cooldown. These symptoms, in combination, pointed to a steam space leak in the pressurizer. A steam space leak refers to steam leaking from the pressurizer. Examples of steam space leaks are leaking pressurizer PORVs and leaking pressurizer safety valves.

To identify the steam space leak, the crews had to recognize that the symptoms on the primary side, specifically pressurizer level going up while pressure continued to go down and the activity in the PRT, could not be explained as the result of known manual or automatic actions. The procedures did not provide any guidance in identifying the steam space leak.

The analysis examined:

- Whether crews detected the symptoms of the leaking PORV;

- How they explained the early symptoms that could be accounted for by the occurrences on the secondary side;

- Whether the crews identified the steam space leak on the primary side.

\section{Situation Assessment: Detecting abnormal plant behavior}

We examined whether crews detected the RCS symptoms that provided evidence of a problem on the primary side.

All the crews observed the pressurizer level going up. In most cases (five of eight) comments on the pressurizer level going up were first made by the RO, who has the responsibility of monitoring and controlling the primary side of the plant. With the exception of Crew $D$, that had closed the pressurizer PORV when the pressurizer level started to go up, the pressurizer either became full or approached it. All but one of the crews (Crew $\mathrm{H}$ ) commented on this.

Six of the eight crews explicitly commented on loss of subcooling. Six of the crews gave a verbal indication of checking reactor vessel level by looking at the reactor vessel level indication system (RVLIS). Seven of the crews explicitly concluded that a bubble had formed in the reactor vessel. Since there were no alarms or procedural directives to check RVLIS, observation of RVLIS was based on knowledgedriven monitoring. Similarly, there were no direct indications of a bubble in the head of the reactor vessel. This situation assessment required an inference based on loss of subcooling and/or the observation of pressurizer level going up, while RVLIS was going down. Crew $\mathrm{H}$ provided no evidence of having detected the loss of subcooling, checked RVLIS, or deduced a bubble in the reactor vessel.

In at least four cases RVLIS level went below $90 \%$. In the case of $\mathrm{Crew} \mathrm{H}$ it went as low as $76 \%$. In no case was the criterion for the core cooling safety function "red path" reached ( $40 \%$ RVLIS).

All the crews identified a problem in the PRT either based on early symptoms (PRT pressure and temperature), or when the PRT ruptured.

\section{Situation Assessment: Explaining observed symptoms}

As described above, the majority of the crews noticed the symptoms providing evidence to a steam space leak. We next examined how the crews explained these symptoms, and whether they correctly identified a steam space leak on the primary side.

We examined the point in the event when a crew member first mentioned primary side plant behavior and the explanation given for the observation. In the case of five of the eight crews the first comment was made by the RO and occurred early in the event when the pressurizer level and pressure were decreasing. At that point the crews were depressurizing the steam generators and a decrease in pressurizer pressure and level was expected due to cooldown. In three of the five cases the crews explicitly attributed the observed decrease to a cooldown. This is an example of a situation where expectations derived from a situation assessment ( a decrease in pressurizer level and pressure due to cooldown) are used to explain observed symptoms.

In three cases (Crews $C, D$, and $H$ ) the crews did not comment on the primary side plant behavior until the level in the pressurizer started to increase and/or the subcooling limit was reached. 


\section{Cognitive Performance}

Table 3.6. LHS 1. Crew identification of a steam space leak.

\begin{tabular}{cccc}
\hline Crew No. & Identified steam space leak & Crew Member & Reason Given \\
\hline A & yes & SO & Level up, pressure down \\
B & no & n/a & n/a \\
C & yes & SO & Level up, pressure down \\
D & PORV Leak & BOP & PRT symptoms \\
E & no & n/a & n/a \\
F & PORV Leak & SS & PRT symptoms \\
H & no & n/a & n/a \\
M & yes & ct & Pressurizer level up \\
\hline
\end{tabular}

Situation Assessment: Identifying a problem -- steam space leak

Identifying a steam space leak required recognizing that some of the observed symptoms could not be explained by the factors the operators knew to be influencing plant behavior, and searching for an explanation that would account for these symptoms.

Table 3.6 shows which of the crews identified a steam space leak and what symptoms led them to that assessment (the 'Reason Given' column). Some of the crews explicitly used the term "steam space leak." Others hypothesized a PORV leak in particular. Those cases are indicated with the label "PORV Leak." Five of the eight crews were able to correctly diagnose a steam space leak from the symptoms observed. In all cases the identification of the steam space leak was based on identification of primary side symptoms that could nor be explained in terms of the known factors influencing plant behavior. In three cases it was when they observed pressurizer level going up while pressure was going down. In two cases it was when they observed PRT symptoms.

While all the crews identified the main symptoms that pointed to a steam space leak (i.e., pressurizer level going up; a bubble in the reactor vessel; PRT symptoms), not all the crews were able to integrate the evidence to identify the steam space leak.

We present protocol segments from two crews to illustrate the complexity of the situation assessment involved, and the variability in crew performance observed. Both crews observed the same symptoms. The first crew was able to integrate the evidence observed to identify the steam space leak relatively quickly. The second crew never identified the steam space leak.

Case 1: Seeking a single explanation to account for all the observed symptoms

The first crew actively sought a single explanation that would account for all of the observed symptoms. This led them to consider the hypothesis of a leaking PORV and to decide to close the PORV block valve in attempting to terminate the leak. As a result they were able to terminate the leak before the pressurizer became full, and before the PRT ruptured, reducing the severity of the incident (i.e., no radioactive fluid spilled into containment).

\section{Seeking a Single Explanation Crew M}

The protocol segment starts at the point where the $R O$ identifies an unexpected behavior in the pressurizer. This leads the SS to identify a steam space leak.

12:05:40 RO: My pressurizer level is screaming. We either just voided something, or something has just happened.

SS: It's coming down?

RO: No, it is screaming up. 
12:05:50 RO: Just lost subcooling. That's why I was concerned.

...

SS: Maybe we sprung a leak.

It's possible.

It would be a leak in the pressurizer, right?

12:06:10 That's right; it would be steam right?

Yea.

12:08:35 SS: For a leak anywhere it's got to be in the steam space of the pressurizer.

SO: Or we are just swapping the bubble?

...

We got pressure coming right on down.

12:09:38 Pressure is decreasing and a bubble is forming.

RO: That's why subcooling went away.

*** At this point the SS explicitly considers the possibility of a leak through the PORV. The SO suggests closing the PORV block valve to test that hypothesis.***

12:09:50 SS: For pressurizer level to do that it's got to be in the pressurizer. Have we lifted a safety? How about a PORV?

RO: We haven't had a PORV lift, right? We may have some leak by, but it is not significant.

12:10:05 SO: You have one of them armed, right? We can go back and block this and see what leaked by.

This valve can really be open for some reason.

What's the PRT?

12:10:37 SO: It looks like a leak on the pressurizer with pressure decreasing and level coming up.

SS: It's a pressurizer leak. It's not going to containment.

RO: It's going into the PRT.
12:10:49 SS: This PORV must have been leaking by. Looks like our pressure in the PRT stopped coming up.

*** The event was terminated shortly after. ***

The protocol segment above provides evidence that the crew used expectations to guide their monitoring of plant behavior, integrated evidence to identify a steam space leak, and identified a response action that would terminate the leak, closing the block valve. The protocol segment also illustrates that multiple crew members contributed to the situation assessment and response identification. In this case the SS, SO, and $\mathrm{RO}$ were all participating.

\section{Case 2: Recognizing an unexplained problem in the RCS}

The next protocol segment is of a crew that recognized there was an unexplained problem in the RCS, but did not identify the steam space leak. This protocol segment is presented to illustrate the difficulty involved in making the situation assessment. The crew noticed all the symptoms pointing to a steam space leak, but were unable to generate a single explanation that would connect all the symptoms. They postulated multiple faults to account for the set of symptoms, and never considered the possibility of closing the PORV block valve to terminate the leak. As a result, plant conditions became more degraded in the case of this crew as compared to the first crew.

\section{Recognizing an Unexplained Problem in the RCS Crew E}

The protocol segment begins with the RO alerting the SO that pressurizer level is up to $75 \%$. This quickly leads them to detect that RCS pressure is decreasing, that they are losing subcooling, and that there is likely a bubble in the head of the reactor vessel.

14:15:30 RO: Well, $\mathrm{SO}$, we are about $75 \%$ on the pressurizer now.

14:15:33 SO: Better get some letdown going. 
14:15:38 RO: Probably voiding somewhere. We couldn't have gone up to that high without voiding.

SO: We are right at saturation.

14:15:54 RO: I got 23 on pumps on and 95 pumps off (subcooling).

SO: Wide range pressure is below a 1000 on the RCS.

14:16:07 SO to STA: You are still monitoring that core cooling?

14:16:17 STA: Yea, ...

$\cdots$

*** At this point pressurizer becomes full and pressurizer pressure is around 800 psig. The RO alerts the SO to this, who suggests actions they can take to attempt to recover RCS pressure. .**

14:17:24 RO: Pressurizer level is at $100 \%$ or greater.

14:17:32 RO: Pressure is a little above 800 .

$\cdots$

${ }^{* * *}$ At this point the RO alerts the crew that the PRT is about to rupture. While they notice the PRT symptoms, they do not integrate them with the symptoms in the pressurizer to conclude a steam space leak. This contrasts with the performance of Crew M. ${ }^{* * * *}$

14:18:28 RO: PRT pressure is screaming up.

SO: From what source?

14:18:35 RO: I don't know but the pressure is now screaming back down. We obviously just blew the rupture disk on the PRT.

14:19:51 SO: Any idea of what the source of water into the PRT is?

14:19:59 RO: No, I'm not really missing any yet.

...

*** The crew monitors RVLIS level and notes that it has decreased significantly.***

14:22:30 SO: What ... is wrong with the PRT? Relief valve some place?
14:24:50 SO: What's the vessel level doing here, STA, you were watching it?

14:24:55 RO: We had 97 in the lowest one. Now we are down to 80 .

SO: The vessel level is dropping?

…

*** At this point the crew considers the possibility of a seal leak to explain containment symptoms. They show no evidence of trying to come up with a single connected explanation to account for all the symptoms observed .***

14:25:22 SO: Seal injection filter delta $P, R O$, they're bouncing.

14:25:40 SO: You got a containment sump level high. Have we blown a seal or something?

$\cdots$

*** The event continued but the crew never identified the steam space leak .***

The contrast in performance of the two crews points out the complexity of the situation assessment involved, and illustrates the variability in crew performance observed. The first crew was able to identify the steam space leak and terminate it before the PRT ruptured. As a result they were able to reduce the severity of the incident. In contrast, the second crew, while observing all the relevant symptoms, was unable to connect the symptoms into a single coherent explanation. They did not identify the steam space leak or attempt to take action to terminate leak. As a result conditions in the RCS continued to degrade. The pressurizer became full, and the PRT ruptured, releasing radiation into containment.

The fact that the first crew was able to terminate the leak and reduce the severity of the incident provides an example of the positive role correct situation assessment can play in mitigating incidents. 


\subsubsection{A Case Where Operators Had to Decide Whether to Manually Initiate a Safety System Based on Consideration and Balancing of Multiple Goals}

All the crews detected symptoms of RCS degradation, and the majority identified the steam space leak on the primary side. The Loss of Heat Sink procedure provided no explicit guidance for dealing with the leak. We examined the options the crews considered for dealing with the leak on the primary side, and their decisions.

For the crews that considered the possibility of a leaking PORV, a viable option for terminating the leak was to close the block valve. Table 3.7 shows which crews explicitly considered the possibility of a leaking pressurizer PORV, the reason given for this hypothesis, and whether they considered closing the PORV block valve to isolate the leak. Five crews hypothesized the possibility of a leaking PORV and considered the option of closing the PORV block valve. Four of the five crews decided to take this action. This decision turned out to be relatively simple because closing the block valve could potentially terminate the leak, and had minimal negative side effects. Only one of the crews (Crew A) decided against closing the block valve. The SO for this crew worried that if he closed the block valve he might not be able to open it later if he needed it. None of the other crews raised that concern.
The way LHS 1 was run the PORV leak was not necessarily terminated when the crew closed the PORV block valve. The leak was terminated in the case of Crew D. In the case of the other crews the leak was continued in order to examine whether the crew would manually initiate SI.

The only procedural guidance available to the operators regarding manual initiation of SI was in a caution that stated: " Following block of automatic SI actuation, manual SI actuation may be required if conditions degrade." This caution allows crews to manually initiate $\mathrm{SI}$ at their discretion.

Manual initiation of SI would recover conditions in the RCS but could result in a delay in recovery of the secondary heat sink or a need to resort to bleed and feed, which is a less desirable way to achieve a heat sink.

If SI is not manually initiated, conditions in the RCS will continue to degrade. Eventually, reactor vessel level (RVLIS) would decrease to less than $40 \%$. At that point, based on a core cooling critical safety function criterion, the EOPs would direct the operators to transition to a procedure designed to respond to loss of core cooling. However, by that point conditions in the RCS would be significantly degraded with increased risk of core damage.

Crew performance was examined for evidence that they recognized that they could manually initiate SI if they determined it was necessary, and for evidence that they considered the multiple goals that needed to be balanced in deciding whether to initiate SI.

Table 3.7 LHS 1. Decisions to close the PORV block valve.

\begin{tabular}{ccccc}
\hline Crew No. & Consider Leaking PORV & Crew Member & Reason Given & Action \\
\hline A & yes & SO & PRT rupture & Do not close block valve \\
B & no & n/a & n/a & n/a \\
C & yes & SO & PRT rupture & Close block valve \\
D & yes & BOP & PRT symptoms & Close block valve \\
E & no & n/a & n/a & n/a \\
F & yes & SS & PRT rupture & Close block valve \\
H & no & n/a & n/a & n/a \\
M & yes & SS & Level up, pressure down & Close block valve \\
\hline
\end{tabular}


Table 3.8 LHS 1. Crews that considered initiating SIor going to a bleed and feed.

\begin{tabular}{cccc}
\hline Crew No. & Consider Sl or Bleed \& Feed & Crew Member & Reason Given \\
\hline A & yes & RO & Containment symptoms; lost \\
B & yes & so & Containment symptoms \\
C & yes & SS & Containment symptoms \\
D & yes & ct & Level up; bubble in head \\
E & yes & SO & Cannot tell; checks procedure options \\
F & yes & BOP & RVLIS coming down \\
H & no & $\mathrm{n} / \mathrm{a}$ & $\mathrm{n} / \mathrm{a}$ \\
M & in the debriefing & $\mathrm{n} / \mathrm{a}$ & In the debriefing: if PRT kept going up \\
\hline
\end{tabular}

Table 3.8 shows whether crews explicitly considered the option of manually initiating SI or going directly to a bleed and feed (which would include a manual SI). Six of the eight crews explicitly discussed the possibility of initiating SI or going to a bleed and feed. A seventh crew (Crew $M$ ) indicated during the debriefing that they would have considered a manual $\mathrm{SI}$, if RCS conditions continued to degrade after they closed the PORV block valve. 35

Table 3.9 shows what decision the crews came to and the reason they gave for their decision. Of the seven crews who considered the possibility, five decided against it. Only one of the crews (Crew B) decided to initiate SI. A second crew (Crew $M$ ) indicated during the debriefing that they would have initiated SI if conditions continued to degrade.

Examination of the reasons given for deciding not to initiate SI indicates that in the majority of cases the crews did not recognize that they were procedurally allowed to initiate SI. Four of the six crews which explicitly considered the possibility of manually initiating SI during the event decided against it because they could not find any procedural guidance directing them to initiate SI.

The caution that appeared in the Loss of Secondary Heat Sink procedure just before the SI signal was blocked was not considered in deciding whether to

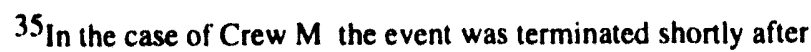
they closed the PORV block valve. As a result they had no chance to observe further degradation of conditions in the RCS.
}

manually initiate SI. The last column in Table 3.9 indicates whether the caution was read aloud at the point when SI was blocked. As can be seen, only four of the eight crews read the caution aloud. None of the crews mentioned the caution in their discussions of whether to manually safety inject or not. In addition, when the caution was explicitly brought up by the instructor during the debriefing, the crews did not believe that it applied in this situation. In general, they did not interpret the caution as permission to initiate SI based on their own judgment.

During the debriefing six of the eight crews indicated that they believed they could not take any action to deal with the degraded RCS conditions until they met an explicit procedure criterion. They interpreted the phrase "conditions degrade" in the caution to mean meeting some explicit procedure criterion that would direct them to turn on SI; specifically, they believed they had to wait until they either explicitly met the bleed and feed criteria, or met the criterion to transition to the core cooling critical safety function procedure ( $40 \%$ RVLIS). Since the event was terminated well before RVLIS reached $40 \%$ it is not possible to know whether the crews would actually have waited for reactor vessel level to decrease below $40 \%$ before manually initiating $\mathrm{SI}$. 
Table 3.9 LHS 1. Crew decioion regarding manual initiation of SI.

\begin{tabular}{cccc}
\hline Crew No. & Decision & Reason Given & Caution Read Aloud \\
\hline A & no & No procedural guidance & yes \\
B & delay & STA says wait & yes \\
C & no & SO says wait & no \\
D & no & No procedural guidance & no \\
E & no & No procedural guidance & no \\
F & no & No procedural guidance & nn \\
H & n/a & n/a & yes \\
M & n/a & n/a & yes \\
\hline
\end{tabular}

The decision of whether to manually initiate $\mathrm{SI}$ is a complex decision that requires consideration of multiple factors. It requires consideration of the multiple goals to be achieved (the goal of terminating the LOCA, the goal of maintaining core cooling, and the goal of recovering a heat sink); alternative means available for achieving those goals and their relative desirability (continuing to try to re-establish feedwater vs. going to a bleed and feed); and the potential costs in increased risk of delays in taking action (the costs of delaying mitigating the leak on the primary side vs. the cost of delay in recovery of feedwater). The crews participating in this event provided little evidence of reasoning about goals in this way in deciding whether to initiate SI.

While the crews expressed concern regarding the degrading conditions in the primary system the main response was to search for an explicit procedure directive to initiate SI. Below, we present a protocol segment of a crew that showed the clearest evidence of considering the severity of conditions in the RCS in deciding whether to initiate SI. This crew, as the others did, searched for an explicit procedure criterion for initiating SI. Failing to find one, they concluded that their best option was to continue efforts to recover feedwater.

\section{Determining Whether to Manually Initiate SI Crew E}

*** This protocol segment begins late in the event, after the PRT has ruptured, a bubble has formed in the head of the reactor vessel and the pressurizer has gone solid. The crew monitors RVLIS level and notes that it has decreased significantly.***

14:24:50 SO: What's the vessel level doing here STA? You were watching it.

14:24:55 RO: We had 97 in the lowest one, Now we are down to 80 .

SO: The vessel level is dropping? (yes).

14:26:48 BOP: Where are we at? At RCS pressure?

RO: 650 Narrow range and it's fluctuating.

14:26:57 SO to STA: You've still been monitoring that core cooling?

STA: Yea.

*** At this point the SO checks whether they meet the EOP criteria for bleed and feed, which is three steam generators less than $24 \%$. They do not meet this criterion. The SO checks the EOP, and concludes that he is procedurally bound to remain in a loop until the bleed and feed criterion is met. ${ }^{* * *}$

14:27:15 SO: Still have wide range level in two of your generators?

14:27:22 BOP: I've got $20 \%$ in $A$ and $B$, and $50 \%$ in Charlie and Delta. 
14:27:30 SO: As far as I can tell, there is nothing that jumps me beyond this step, wide range level less than $24 \%$ in three of the steam generators. It jumps you to step 10 (bleed and feed step).

...

${ }^{* * *}$ At this point the $R O$ reminds the $S O$ that containment radiation is increasing.

14:29:17 RO: Containment radiation is going up. We have $1000 \mathrm{mr}$ in the person hatch.

...

*** At this point the SO provides evidence that he has considered options for dealing with the unexplained problem in the RCS, and has decided that his best course of action is to continue attempts to recover feedwater and postpone action with respect to the RCS problem. While he recognizes that conditions in the $R C S$ have degraded considerably (RVLIS level is less than $80 \%$, and they have a "yellow" path on core cooling), he decides that conditions are not severe enough to take action. ***

14:31:10 SO: It looks to me we are not having any problem with the core right now. There's something we can't account for over here but it is not causing any possibility of a core melt or excess temp or anything. We still have core cooling. We have a problem but it is not yet critical. The next most important thing is get a heat sink. I'd rather stay in here and get that if there is any way of doing it.

*** At this point the SO exhibits the mark of an important crew interaction skill - "openness." He polls the crew for alternative opinions and seeks consensus before taking action.***

14:31:40 SO: Are you in agreement with me? I'm open to discussion on this. (They all agree.)

14:31:46 STA: Still yellow on core cooling.

14:31:55 SO: OK, we are yellow on core cooling; we're green on subcriticality; we're still red on heat sink.

14:32:10 RO: And we are at $1300 \mathrm{mr}$ at the personnel hatch.

14:32:15 SO: I still need you to keep looking; if you have a leak where it is at.
This protocol segment illustrates several points. First, the protocol segment provides evidence that the SO was considering multiple goals in deciding how to respond to the situation. The crew monitored core cooling and considered the state of conditions in the RCS as well as the heat sink problem in deciding how to respond to the situation. Second, the protocol illustrates that the crew searched for explicit procedural guidance with respect to initiating SI. In this case the SO concluded that he was procedurally bound to stay in the Loss of Heat Sink procedure until feedwater was recovered, he met the bleed and feed criteria, or he met the red path criteria for the core cooling safety function. Discussions during the debriefing support this interpretation.

\subsubsection{Cases Where Evaluating the Procedure Path Enabled Operators to Catch Their Own Errors}

In the Loss of Heat Sink event we observed three cases where crews got to a step in the EOP that called for a transition to a different procedure, but failed to make the transition. Instead, they continued to go on with the steps in the procedure. In all three cases the crews recognized their error within two steps. These cases provided a concrete example of a situation where evaluating the procedure path enabled crews to catch and correct their own errors.

The three cases arose in the step in the $\mathrm{E}-0$ procedure that transitioned to the Reactor Trip Response procedure if SI was not required. The procedure step first checked if SI was actuated. In the LHS event SI was not actuated. If SI was not actuated, according to the EOP rules of usage, the crews were required to go to the RNO column in the procedure. The RNO column had the crews check if SI was required. In the event we ran, SI was not required. In this case the EOP step directed the crews to transition to the Reactor Trip Response procedure.

Since the same transition occurred in both LHS 1 and LHS 2 we examined the performance of crews in both events. Of the ten crews that participated in LHS 1,36

\footnotetext{
36 The ten crews in LHS 1 included two crews that were dropped from the main analysis because an inadvertent Sl occurred during RCS depressurization.
} 
eight correctly went to the RNO column and from there transitioned to the Reactor Trip Response procedure. Two crews failed to go to the RNO column and continued to the next step in the E-0 procedure. Of the ten crews that participated in LHS 2 , one continued to the next step in E-0 instead of going to the RNO column.

In all three cases the crews caught their error two steps later when they got to a step that asked them to take an action that they knew was not appropriate to the situation. This led them to recognize they were on the wrong procedure path, retrace their steps, and find the point where they had missed the transition to the Reactor Trip Response procedure.

Specifically, the crews realized they were on the wrong procedure path when they got to a step that asked them to verify Containment Isolation Phase A. Containment Isolation Phase A is something that occurs automatically upon actuation of SI. The crews knew that SI had not actuated, and they knew that Containment Isolation Phase A was not needed. This led the crews to recognize that they were on the wrong procedure path. At that point the crews moved back in the EOP and reread the SI actuation step. They recognized that they had failed to go to the RNO column, corrected their error, and transitioned to the Reactor Trip Response procedure.

A protocol segment from one of the crews that missed the transition to the Reactor Trip response procedure and then reversed themselves (Crew J) is provided below.

\section{Catching an Error by Evaluating the Procedure Path Crew J}

*** When they get to step 4 that checks for SI actuation, they correctly indicate that SI did not actuate, but instead of going to the RNO column they move on to step 5 that verifies feedwater isolation. ${ }^{* * *}$

10:12:00 step 4 -- $\mathrm{SI}$ is not actuated yet.

10:12:20 step 5 -- Feedwater isolation.
*** When they get to step 6 that verifies Containment Isolation Phase $A$ (CISA), the RO recognizes that Containment Isolation Phase $A$ is not appropriate to the situation and tells the SO who then realizes he is on the wrong procedure path. ***

10:12:40 step 6 -- Ensure CISA.

RO: Wait a minute we don't have a CISA.

SO: Oh, I'm sorry, you are right, let me back up here.

${ }^{* * *}$ At that point the SO backs up to step 4, goes to the RNO column, checks that SI is nct required, and correctly transitions to the Reactor Trip Response procedure .***

The three crews that missed the transition to the Reactor Trip Response procedure and then caught their error, provide examples of the role of situation assessment and response plan monitoring in catching and correcting errors. In all three cases the crews caught their error because they realized that the actions specified in the procedures were not appropriate to the situation as they understood it. This led them to realize they were on the wrong procedure path and to retrace their steps in search of the missed transition that would get them on the correct procedure path.

\subsubsection{Variability in Crew Performance}

While most of the crews performed well in the LHS 1 event, there was variability in performance. All the crews detected the main plant symptoms that indicated a steam space leak, but not all were able to integrate the evidence correctly. Only five of the eight crews correctly identified the steam space leak.

One of the crews ( $\mathrm{Crew} \mathrm{H}$ ) never realized there was a leak in the RCS. While this crew observed degrading conditions in the RCS (e.g., a bubble forming in the core; pressurizer level increasing), they attributed the behavior in the RCS to the fact that letdown was not in service. 37 In spite of the fact that conditions in

37 While the fact that letdown was not in service might explain an increase in pressurizer level, it could not explain the bubble forming in the core. 
the RCS degraded considerably (RVLIS less than $80 \%$; RCS pressure down to 500; 50 degrees superheated), they didn't realize that in addition to the fundamental, serious problem in the secondary side, they also had a fundamental serious problem in the primary side, i.e., the leaking pressurizer PORV.

The variability in crew performance indicates that identifying the leaking pressurizer PORV was cognitively difficult. It required recognizing that the primary side behavior could not be explained by known factors influencing the plant (e.g., net charging; the cooldown resulting from depressurizing the steam generators), and searching for a coherent explanation that would account for all the symptorns observed. The crews were not all equally successful in these activities.

Differentiating expected from unexpected primary side behavior requires having an accurate mental model of the factors that influence primary side behavior and the size and direction of effect of each of these factors. It also requires qualitative reasoning to determine expected primary side behavior based on the net effect of the known influences affecting primary side behavior at the time. The fact that not all crews recognized that the degrading conditions in the RCS were unexpected given the known factors influencing the RCS suggests that there is room for improving operator knowledge and skills in these areas.

\subsection{Loss of Heat Sink 2: Total Loss of Secondary Heat Sink (Feedwater Recovered)}

As in the case of the LHS 1 scenario, LHS 2 involved a total loss of feedwater flow complicated by a leaking pressurizer PORV. There were two main differences between the two scenarios. One difference is that if a crew decided to close the PORV block valve the pressurizer leak was terminated. This aspect provided the opportunity to examine crew response to degrading RCS conditions in cases where there was no leak as well as in cases where there was a leak. A second difference between the two scenarios is that in LHS 2 the crews eventually recovered feedwater. As a result they transitioned to the Reactor Trip Response procedure. This procedure introduced a new source of cognitive complexity because it included steps that were not appropriate to the situation.

The analysis identified two cases where operators had to engage in situation assessment and response planning in order to deal with the situation. The first case involved identifying and explaining the degrading conditions in the RCS. As in LHS 1 this entailed discriminating plant behavior that was the result of known factors (i.e., an operator induced cooldown) from plant behavior that signaled an additional plant fault (i.e., the leaking pressurizer PORV).

A difference between LHS 1 and LHS 2 is that in LHS 2 if a crew decided to close the PORV block valve the leak was terminated. Eight of the 10 crews run in the LHS 2 scenario terminated the leak by closing the PORV block valve relatively early in the event. This provided us the opportunity to examine crew response to degrading $\mathrm{RCS}$ conditions in cases where there was no leak as well as in cases where there was a leak.

Even when there was no leak on the primary side, RCS conditions degraded. RCS pressure went down to less than $1830 \mathrm{psig}$, and pressurizer level went to less than $4 \%$. These decreases were due to the cooldown cause 1 first by the depressurization of the steam generators, and later by the feeding of the steam generators through the condensate system. The fact that pressurizer level and pressure decreased to this extent just due to the cooldown made clear the cognitive difficulty faced by crews in both LHS 1 and LHS 2 in distinguishing expected pressurizer behavior explained by a cooldown from abnormal behavior that indicates a leak.

The second case provided a concrete example of where actions specified in a procedure are not appropriate to the situation. The Reactor Trip Response procedure included several steps that were potentially inappropriate to follow literally given that the crews had just recovered feedwater using the condensate system. This included steps, both in the body of the procedure and on the foldout page, that specified that the operators should initiate SI. The EOP background documents anticipated the possibility of steps being inappropriate and explicitly indicated that operator judgment may be needed 
under these circumstances. 38 This situation allowed us to examine operator performance in a case where operators were required to evaluate the appropriateness of procedure steps given the specifics of the situation and to modify the steps if judged necessary.

The analysis focused on whether crews chose to deviate from the procedure steps that were judged to be inappropriate and the basis for their decision. We had the opportunity to examine crew response to procedure steps that called for manually initiating SI in both cases where there was no leak present in the $\mathrm{RCS}$ and cases where there was a leak.

\subsubsection{Characteristics of Participating Crews}

Ten crews participated in the Loss of Heat Sink 2 scenario. Four of the crews were staff and six were currently on shift. In the case of two of the staff crews (Crews 1 and 5) one or more crew members were aware of the event. In Crew 1 the SS had prior knowledge of the event.

In the case of Crew 5 three of the four crew members were "confederates" in that they were fully aware of the event. Only the SO did not know the event. This crew was not dropped from the study because the three confederates did an exceptionally good job of providing the $\mathrm{SO}$ with the information on plant state he would need to identify the leaking PORV, without integrating the information for him. As a result it provided the opportunity to observe the situation assessment and response evaluation activities of the

38 The Users Guide for the Westinghouse Owners Group Emergency Response Guidelines and Background Document explicitly addresses the type of situation created in LHS 2. It states "After restoration of any Critical Safety Function from a RED or ORANGE condition, recovery actions may continue when the FRG is complete... Upon continuation of recovery actions, some judgment is required by the operator to avoid inadvertent reinstatement of a RED or ORANGE condition by undoing some critical step in a Function Restoration Guideline." (Westinghouse Owners Group Emergency Response Guidelines, Users Guide for Emergency Response Guidelines and Background Documents, September 1, 1983, pg. 17.) The use of the phrase "some judgment is required by the operator" suggests that the developers of the EOPs recognize that in these circumstances operators need to evaluate the appropriateness of certain procedure steps based on their own situation assessment.
SO. In this case the focus of study was the situation assessment and decision-making activities of a single individual rather than a group.

\subsubsection{A Case Where Operators Needed to Determine Whether Plant Behavior was the Result of Known Manual and/or Automatic Actions or the Result of a Plant Fault}

Situation Assessment: Identifying a problem -- leaking
pressurizer PORV

The analysis examined whether crews identified the leaking pressurizer PORV. Table 3.10 lists the crews that closed the block valve, the reasons they gave for closing it, and whether they explicitly suspected a leaking PORV when they closed the block valve. Of the ten crews who participated in the event, three crews closed the PORV block valve at the time that they closed the pressurizer PORV when RCS was depressurized to less than 1920 psig. ${ }^{39}$ As a result, these three crews never experienced a leaking PORV.

Of the remaining seven crews, five crews suspected a leaking pressurizer PORV and closed the block valve as a result. Three of these crews suspected a leaking pressurizer PORV because of a rapid decrease in pressurizer pressure. The other two crews suspected a leaking pressurizer PORV when they got PRT alarms.

Two of the crews (Crew 5 and Crew 11) never suspected a leak in the PORV. As a result, the leak was still present when they transitioned to the Reactor Trip Response procedure.

\footnotetext{
39 One possible reason that three of the ten crews closed the block valve at the time the PORV was closed is that at this plant the block valve is normally closed below 2185 psig. The operators opened the block valve as part of the RCS depressurization, but then returned it back to its original configuration after the RCS target pressure was reached.
} 
Table 3.10 LHS 2. Whether crews closed the pressurizer PORV block valve and their reason for closing it.

\begin{tabular}{ccccc}
\hline Crew No. & Close Block Valve & Crew Member & Reason Given & Suspect PORV Leak \\
\hline 1 & yes & RO & Pressure down & yes \\
2 & yes & RO & PRT symptoms & yes \\
3 & yes & RO & PRT symptoms & yes \\
5 & no & n/a & n/a & no \\
6 & yes & RO & Pressure down & yes \\
7 & yes & RO & When close PORV & n/a \\
8 & yes & RO & Pressure down & yes \\
9 & yes & BOP & When close PORV & n/a \\
10 & yes & BOP & When close PORV & n/a \\
11 & no & BOP & n/a & no \\
\hline
\end{tabular}

Situation Assessment: Identifying unexpected plant behavior

The variability in performance across crews indicates that identifying the leaking pressurizer PORV was cognitively challenging. It required discriminating the effects of a leak from effects of other known influences on RCS behavior, such as the cooldown that resulted from activities on the secondary side.

The following protocol segment illustrates the difficulty of discriminating expected from unexpected plant behavior in this event. In this protocol segment a crew at first attributes the RCS behavior to a cooldown, when in fact there is also a leak present. Eventually, they get alarms indicating a problem in the PRT from which they infer that the pressurizer PORV must be leaking. At that point they reassess the situation and conclude that the RCS symptoms they have been observing were at least in part due to the leak.

\section{Difficulty of Discriminating Effects of a Leak from Effects of a Cooldown}

Crew 2

The crew observes that pressurizer level is decreasing, RCS pressure is decreasing and subcooling is decreasing. At first they attribute it to a cooldown.
10:07:35 RO (to SO): RCS pressure ... we are getting closer to the unacceptable region on the subcooling curve but with him dumping as much steam we should come back ... pretty quickly.

...

10:08:03 RO: Due to the cooldown my pressurizer level is decreasing.

SO: I understand.

10:08:11 RO: $20 \%$ on pressurizer level, I don't think that's an uncontrolled decrease.

10:08:47 RO: Just got less than $17 \%$.

10:08:58 SS: It's shrinking you down.

*** At this point, while they continue to believe the RCS symptoms are due to a cooldown they begin to take actions to try and recover level. In this case they start a centrifugal charging pump. This has the effect of introducing yet another factor influencing RCS behavior, making it more difficult to sori out all of the known influences affecting $R C S$ and distinguish expected from unexpected RCS behavior. ***

10:09:00 RO: Do you want me to start the CCP?

10:09:10 SO: Agrees. No sense taking pressurizer level down and out of sight. 
10:09:59 RO: Taking manual control of PDP speed controller and the CCP flow control valve and I will secure the PDP.

\section{0:10:00 SO: I understand ...}

...

*** At this point they get a PRT level high alarm which leads the RO to suspect a steam space leak. and close the pressurizer PORV block valve. ***

10:12:10 RO: PRT level high (acknowledges alarm).

10:12:16 RO: I'm going to close the pressurizer seal iso block valve for possible leakage pathway; that PRT level has continued to increase.

*** The SO reassesses the situation and concludes that the pressurizer level decrease must have been in part attributable to the leak in the pressurizer. The $R O$ agrees. ***

10:12:27 SO: You mean that may be part of your level decrease?

RO: Yes.

$\cdots$

10:14:39 RO: Pressurizer level has turned and it is recovering. I'm still putting in approx. $200 \mathrm{gpm}$ charging.

This protocol segment illustrates that the crew was engaging in situation assessment. They showed evidence of maintaining a model of the factors influencing RCS behavior and using that to explain observed RCS behavior. At first they explained RCS behavior by the cooldown caused by activities on the secondary side. Only after they identified an independent symptom that pointed to a pressurizer leak (PRT level high) did they update their model of the factors influencing RCS behavior.

The protocol also illustrates the difficulty of detecting the influence of a small leak in RCS when there are a number of other influences on the RCS at the same time. The behavior of the RCS exhibited at this point -- decrease in pressurizer level less than $17 \%$, decrease in pressurizer pressure, decrease in subcooling margin - was not very different from the RCS symptoms exhibited in cases where there was no leak.

The difficulty of the required discrimination was compounded by the fact that the crews were constantly taking action that changed the pattern of influences on the RCS. For example, they turned on heaters, started pumps, and isolated letdown, in an effort to bring pressurizer level and pressure back up. These actions increased the difficulty of predicting what the RCS behavior should be and detecting discrepancies.

Detecting the leak in the RCS required qualitative reasoning comparing the expected decrease in RCS parameter values due to known ongoing influences with the observed decrease. Since crews do not get much experience with attempts to provide feedwater through the condensate system, they did not have much basis with which to predict the size of shrink to expect due to the cooldown caused by the rapid depressurization of the SGs and subsequent start of feedwater via the condensate system.

The need to discriminate cooldown effects from effects due to a leak on the primary side is reinforced by the behavior of the crews once the leak was terminated. Table 3.11 shows the first point at which crews considered manual initiation of SI in the scenario, the procedure they were in at the time, and the basis for their concern, whether they decided to initiate SI, and the basis for their decision. As can be seen, seven of the crews were sufficiently concerned with primary side behavior that they explicitly considered manual SI at least once in the scenario. In the case of six of these seven crews there was no leak present. 40 The degraded RCS conditions were due to a cooldown. After checking for additional evidence of a leak and discussions among the crew members, these crews eventually (correctly) decided that the RCS behavior was explained by a cooldown and decided not to initiate SI.

The difficulty of the discrimination is highlighted by the fact that Crew 11 also decided against manually initiating SI the first time the possibility was raised. In their case there was a leak present, and initiating SI would have been appropriate.

\footnotetext{
40 There was a leak in the case of Crew 11.
} 
Cognitive Performance

Table 3.11 LHS 2. First point at which crews considered manual SI.

\begin{tabular}{cccccc}
\hline Crew No. & Procedure & Crew Member & Reason Given & Decision & Reason Given \\
\hline 1 & Reactor Trip & RO & Przr level $<4 \%$ & no & Level inc; SS says not to \\
2 & n/a & n/a & n/a & n/a & n/ \\
3 & LHS & RO & Przr level down & no & SS says controlled cooldown \\
5 & n/a & n/a & n/a & n/a & n/a \\
6 & n/a & n/a & n/a & n/a & n/a \\
7 & Reactor Trip & RO & Przr level $<4 \%$ & no & Operator induced \\
8 & LHS & RO & Przr level down & no & Try to stabilize \\
9 & Reactor Trip & RO & Przr pressure & no & Due to cooldown \\
10 & Reactor Trip & RO & Przr pressure & no & Operator induced \\
11 & Reactor Trip & RO & Przr pressure & no & SS says not due to a leak \\
\hline
\end{tabular}

Response Planning: Reading and interpreting caution in the Loss of Heat Sink procedure

We also examined whether crews read aloud the caution regarding manual activation of SI that appeared prior to the step in the Loss of Heat Sink procedure where SI signals were blocked. Nine of the ten crews read the caution aloud. Two of these crews (Crew 6 and Crew 8) explicitly mentioned the caution when they had concerns about RCS conditions and were considering courses of action.

The results regarding reading and interpreting the caution contrast with the results on LHS 1 . In that case operators indicated that they interpreted "conditions degrade" as degrading severely enough to meet explicit EOP transition criteria (i.e., bleed and feed criteria or RVLIS less than $40 \%$ ). In the case of LHS 2, which was run at a different plant, some of the crews considered pressurizer level less than $17 \%$ to be a degraded condition warranting consideration of manual SI. The wide variability in interpretation of the phrase "conditions degrade" suggests that clarification of the intent of that statement may be required.

\subsubsection{A Case Where Operators Were Required to Evaluate the Appropriateness of Procedure Steps Given the Specifics of the Situation}

Because feedwater was recovered in LHS 2, we were able to observe the crew's actions when they transitioned to the Reactor Trip Response procedure and reached steps that seemed inappropriate to the situation. Of the ten crews that participated in the event, one crew (Crew 2) had the scenario terminated before they got to the reactor trip procedure, and a second crew (Crew 8) manually initiated SI based on the foldout page criterion of pressurizer level less than $4 \%$ as soon as they entered the procedure.

This left eight crews that went through the Reactor Trip procedure. Of those, six of the crews had terminated the leak through the pressurizer PORV before they entered the Reactor Trip procedure and two crews still had a leak. This difference allowed us to examine how crews responded to steps in the Reactor Trip procedure that called for an SI, both in cases where there was no leak and an SI would be inappropriate, and in cases where a leak still existed and an SI was required.

The decision of whether to initiate SI was important because either course of action potentially had negative consequences if the crew's situation assessment turned out to be wrong. If they decided to 
safety inject when it was not needed, it could place the crews in a loss of feedwater event again or at the minimum delay recovery. If they decided to not initiate SI, and there was a leak in progress, conditions would continue to degrade, with increased risk.

\section{Response Planning: Omitting a procedure step considered inappropriate to the situation}

Table 3.12 shows how crews responded to the steps in the Reactor Trip procedure that were not appropriate given that feedwater was being provided through the condensate system. One step required that the operators close the feedwater isolation valves. Crew response to that step are presented in the column labeled "Omit Feed Isol. Valve Step" in Table 3.12. A 'yes' in that column indicates that the crew decided to omit the step and not close the isolation valve. Since the crews had intentionally opened the feed isolation valve as part of the Loss of Heat Sink procedure, and closing that valve would result in a loss of feedwater again, the crews had no difficulty deciding not to close that valve. All eight crews decided to leave the feedwater isolation valve open. In most cases the decision was made by the $\mathrm{SO}$ with no discussion required. Examples of explanations given by the $\mathrm{SO}$ for the decision are "We opened them intentionally, I am not going to close them again" (Crew 6); and "We are not going to do that because that is how we are feeding the generators" (Crew 10). ${ }^{41}$

The second step that had to be adapted had operators check that pressurizer pressure was greater than 1830 , and initiate manual SI if pressurizer pressure was lower. Pressurizer pressure was less than 1830 in the case of all seven crews that reached that step. In the case of five of the crews this was primarily due to cooldown. However, in the case of two of the crews there was a leak in the RCS that contributed to the low pressurizer pressure.

Column 4 in Table 3.12 labeled "Omit Przr Press. step" shows crew performance on the step that required

\footnotetext{
41 In this scenario none of the crews made the mistake of following a procedure step that undid an action that was taken earlier to restore a critical plant function. There is anecdotal evidence of an instance where a crew did just that during a training exercise at a plant.
}

crews to SI if pressurizer pressure was lower than 1830. A 'yes' in that column indicates that the crews decided to omit the procedure step and not initiate SI. The next column labeled 'who decides' indicates which crew member made the decision. A "SO w/SS" indicates that the decision was made by the $\mathrm{SO}$ and approved by the SS. 42

As can be seen all seven of the crews that reached that step decided against manual initiation of SI. In most cases the decision was not made unilaterally by the SO, but involved input from other crew members and agreement by the SS.

While all crews came to the same decision regarding whether to initiate SI based on pressurizer pressure less than 1830, the decision was not necessarily correct in all cases. In the case of two of the crews (Crews 5 and 11), there was an ongoing leak in the PORV. The decrease in pressurizer pressure was not entirely due to a cooldown as they assumed. This result re-emphasizes the need for crews to be able to distinguish effects of leaks from effects of cooldown, and the difficulty of making this discrimination.

In addition to explicit procedure steps, a foldout page in the Reactor Trip procedure specifies criteria for manually initiating SI. Steps on the foldout page are intended to apply at all times and the actions specified are supposed to be taken as soon as the criteria are met. Table 3.13 shows which foldout page SI criterion was met, whether there was a leaking PORV at the time, whether the crew decided to initiate SI , who made the decision, and the reason given.

There was one SI criterion specified on the foldout page that was met even in cases where there was no leak. This was the criterion of pressurizer level less than $4 \%$. In several cases pressurizer level reached zero strictly because of the on-going cooldown. Five crews met the less than $4 \%$ level SI criterion. In none of these cases was there a leak. One crew (Crew 8) immediately safety injected based on the foldout page criterion. In the case of the other four crews, three concluded the level decrease was due to an operator induced cooldown and decided against SI. The fourth crew (Crew 9) never considered this SI criterion. The 42 'SO w/SS, STA' indicates that the decision was made by the SO
and approved by the SS and STA. 
Cognitive Performance

Table 3.12 LHS 2. Crew response to Reactor Trip procedure steps that required modification.

\begin{tabular}{ccccc}
\hline Crew No. & Omit Feed Isolation Valve Step & Crew Member & Omit Przr Press. Step & Crew Member \\
\hline 1 & yes & SO & n/a & n/a \\
2 & yes & n/a & yes & SO w/SS \\
3 & n/a & n/a & n/a & n/a \\
5 & yes & so with SS & yes & SO w/SS \\
6 & yes & SO & yes & SO \\
7 & yes & SO & yes & SO w/SS,STA \\
8 & n/a & n/a & n/a & n/a \\
9 & yes & SO & yes & SO \\
10 & yes & SO & yes & SO w/SS,STA \\
11 & yes & SO & yes & SO w/SS \\
\hline
\end{tabular}

Table 3.13 LHS 2. Foldout page SI criteria met and crew response.

\begin{tabular}{ccccccc}
\hline Crew No. & SI Criterion Met & Leak & Initiate SI & Crew Member & Reason Given \\
\hline 1 & level $<4 \%$ & no & no & SO w/SS & Level inc., SS says no \\
2 & n $/ \mathrm{a}$ & no & n $/ \mathrm{a}$ & $\mathrm{n} / \mathrm{a}$ & $\mathrm{n} / \mathrm{a}$ \\
3 & $\mathrm{n} / \mathrm{a}$ & no & $\mathrm{n} / \mathrm{a}$ & $\mathrm{n} / \mathrm{a}$ & $\mathrm{n} / \mathrm{a}$ \\
5 & subcooling & yes & no & no decision & \\
6 & $\mathrm{n} / \mathrm{a}$ & no & $\mathrm{n} / \mathrm{a}$ & $\mathrm{n} / \mathrm{a}$ & $\mathrm{n} / \mathrm{a}$ \\
7 & level $<4 \%$ & no & no & $\mathrm{SO} \mathrm{w} / \mathrm{SS}, \mathrm{STA}$ & Operator induced \\
8 & level $<4 \%$ & no & yes & SO w/SS & Foldout page criterion \\
9 & level $<4 \%$ & no & no & SI not considered & n/a \\
10 & level $<4 \%$ & no & no & SO w/SS,STA & Operator induced \\
11 & subcooling & yes & yes & SO & Foldout page criterion \\
\hline
\end{tabular}

variability in response of the crews to this foldout page criterion re-emphasizes the difficulty of the discriminations and decisions the crews confronted.

In the case of the two crews where there was an ongoing pressurizer PORV leak (Crews 5 and 11), the low pressurizer level criterion was not met in the Reactor Trip response procedure because by that point a bubble had formed in the reactor head and pressurizer level was increasing. These crews both met the loss of subcooling SI criteria however. In the case of one of the crews (Crew 11) the loss of subcooling was identified by the STA and the crew manually initiated SI. In the case of the other crew (Crew 5), four of the five members of the crew were confederates who were aware of the event. These crew members informed the SO that pressurizer level was going up, that pressure was continuing to go down, and that subcooling was less than 30 degrees (the SI criterion), without integrating the evidence for the SO, or pointing out that the foldout page SI criterion was met. In this case the SO did not identify the leak nor realize that a foldout page SI criterion was met by the time the simulation was terminated. It is likely that had the simulation continued he would have recognized the need for a manual SI.

The contrast in performance between Crew 11 that recognized that the SI foldout page criteria were met, and Crew 5 that did not, may be due to the difference 
in number of crew members actively participating in situation assessment and response evaluation. It provides some evidence for the positive contribution of multiple crew members to situation assessment and response evaluation.

\section{Situation Assessment: Checking for evidence to confirm} hypothesis; SI is not needed

Many of the crews that correctly decided against manually initiating SI as required by the procedure provided evidence that they considered the intent behind the procedure step, engaged in knowledgedriven monitoring to ensure that the problem situation was not present, and considered the consequences of initiating SI before deviating from the procedure step. In addition, in most cases, they sought consensus among crew members before taking the action.

Protocol segments from two crews illustrate the cognitive difficulty of the decision involved and the types of extra-procedural activities the crews engaged in before deciding whether to deviate from the procedure. In both cases there was no leak present in the RCS.

Case 1: Deciding whether to initiate SI based on a foldout page criterion

In the first protocol segment the crew had to decide whether to manually SI based on the foldout page criterion of pressurizer level less than $4 \%$ :

\section{Decision on Initiating SI Based on a Specific Criterion Crew 7}

*** The SO observes that pressurizer level is below the foldout page criterion for initiating SI and calls the SS over for consultation. ${ }^{* * *}$

10:28:20 SO to SS: I have a question regarding foldout page. If pressurizer level cannot be maintained greater than $4 \%$, then SI. Pressurizer level reads 0 on one meter and $2 \%$ on two meters.

*** The STA comes over to join SS and SO and suggests that the pressurizer level behavior can be explained by an operator induced cooldown and that consequently there was no need to safety inject. He argues that an SI would not help the situation. He suggests that they check other parameters in assecsing whether SI is needed. The SO agrees and tells the $R O$ to monitor pressurizer level and if it looks like it cannot be maintained they will initiate a manual SI. ***

10:29:17 STA: That's operator induced.... if level is increasing, I think we could safely stay where we are at.

Subcooling is greater than $70 \mathrm{deg}$.

10:30:10 STA: I don't think that doing a SI right now would do us any good.

SO: I agree, but by the letter of the law I don't want to get in trouble here.

STA: I say we all talk about it and know what is happening, and look at all the other indications.

10:30:34 SO (to RO): If it looks like we can't maintain. level let me know and we will safety inject.

*** Eventually pressurizer level comes back up, confirming the crew's assessment of the situation ***

10:33:49 RO: Level in the pressurizer is increasing. It is about $6 \%$.

Case 2: Deciding whether to initiate SI based on pressurizer pressure less than 1830.

The next protocol segment is of a crew that has to decide whether or not to manually SI based on a step in the Reactor Trip Response procedure regarding pressurizer pressure less than 1830 .

\section{Decision on Initiating SI Based on Low Pressurizer Level Crew 10}

*** At this point in the scenario the crews observe that they have lost pressurizer level and attributed it to cooldown. ${ }^{* * *}$ 
10:40:12 SO: We lost pressurizer level. We cooled off way fast.

We are recovering real slow.

*** At this point the SO gets to the step that checks that pressurizer pressure is greater than 1830 psig. It is not, so he goes to the RNO column which indicates that SI should be initiated. The SO indicates that he does not want to SI. The RO concurs, pointing out that RCS pressure is stable. ***

10:41:45 Step 5 - Check PRZR pressure greater than 1830 - no.

10:42:30 Goes to RNO -- Verify SI actuation. --

SO: I don't think we want to do that.

10:42:40 BOP: RCS pressure is stable right now.

RO: No, we don't want to SI. Where do you see that?

10:42:56 *** SO, RO and BOP all look at the procedure. ${ }^{* * *}$

*** At this point the SO consults with SS who concurs that SI is not needed. He indicates that the low pressurizer pressure is 'operator induced' meaning that it is due to a cooldown brought on by the activities of the operators on the secondary side. The STA agrees. ***

10:43:06 SO to SS: I need your opinion. I'm at this step it says, ... Verify SI actuation... We don't want to do that.

SS: This is an operator induced thing... we will go on with this procedure in doing the steps we can.

10:44:29 SS confers with STA: I don't think we want to SI.

10:44:40 STA: We are here because we put ourselves here, so it is a controlled action.

*** The RO asks the crew to check that there are no other indications of an RCS leak. The STA checks a number of parameters and reports back that they all read normal .***

10:45:43 RO: We don't have any indications of an RCS leak, right gentlemen? (Others confirm that no, they did not see any indications of a leak.)
10:45:50 STA: RCP seals indicating proper, containment pressure maintaining zero, area rad monitors, I'll check those. Area rad. monitors in containment stable.

...

10:46:54 STA: Natural circ. RVLIS indicates $100 \%$.

10:49:10 We have subcooling of 60 on one and 20 on the other.

*** At this point someone points out that if they did activate SI it would have negative consequences since it might cause them to lose the feedwater they had just recovered. ***

10:49:22 If we activate SI we will lose all feed.

10:49:31 Now it is coming back.

10:49:43 RCS pressure is hanging around $1000 \mathrm{lb}$.

These two protocol segments illustrate that in deciding against manual initiation of SI the crews engaged in several extra-procedural activities. First, they sought to explain the observed RCS behavior. In both cases they determined that the RCS behavior could be explained by known influences. Both crews mentioned that the RCS behavior was "operator induced"; that is, that it was due to the cooldown that they initiated. Second, they explicitly checked to make sure there were no other symptoms of a fault present in the RCS. Based on these observations and situation assessment they determined that a manual SI was not needed. Third, they judged that taking the action (i.e., initiating a manual SI) would have negative consequences. Finally, the crews engaged in these situation assessment and response evaluation activities as a group and sought consensus in the decision.

The results highlight the role of group interaction in situation assessment and response evaluation. The protocol segments presented above illustrate that 
multiple crew members contributed evidence and opinion in formulating situation assessments and evaluating response options. This was particularly true when the crews reached points in the EOP where their assessment of the correct action to take diverged from the actions specified in the procedure. At those points the SOs generally sought input from all crew members and approval from the SS before deciding to diverge from a procedure step.

\subsubsection{Variability in Crew Performance}

The majority of crews correctly identified the leaking PORV and closed the block valve. When they returned to the Reactor Trip Response procedure they correctly judged that SI was not needed and modified the Reactor Trip Response procedure accordingly.

Nevertheless, variability in performance was observed.

Two of the ten crews that participated in the scenario had trouble identifying the leaking pressurizer PORV. Crew 5, where four of the crew members were confederates, had not identified the leak by the time the simulation was terminated. Crew 11 believed the RCS symptoms were due to a cooldown for a long period of time. In particular, when they first raised the possibility of manually initiating SI due to low pressurizer pressure, they decided against it, explicitly indicating that they did not believe they had a leak on the primary side. This crew did decide to manually SI at a later point in the event based on meeting the loss of subcooling SI criteria.

The fact that two crews had trouble identifying the leaking pressurizer PORV suggests that discriminating between RCS behavior due to a cooldown and RCS behavior due to a LOCA was a difficult cognitive task. Sources of complexity included (1) the fact that manual and automatic actions were constantly occurring that changed the pattern of influences on the RCS, making it difficult to predict RCS behavior and discriminate expected from unexpected behavior, and (2) the fact that the crews had little experience with feeding on the condensate system, so they had little basis to judge what the expected effect of the cooldown on RCS behavior would be. 


\section{Crew Interaction in the Simulated Emergencies}

Section 3 focused on the role of higher-level cognitive activity in guiding operator performance in the simulated emergencies. We also examined crew interaction in handling these cognitively demanding scenarios.

Team interaction skills play an important role in crew performance in complex dynamic situations (cf., Swezey and Salas, 1992). Under a separate program sponsored by the U.S. NRC, Montgomery et al. (1992) identified six dimensions of team interaction skill and developed BARS scales for measuring crew performance on those dimensions. The dimensions identified were: (1) communication, (2) openness, (3) coordination, (4) team spirit, (5) task focus, and (6) adaptability. As part of the present study we examined crew performance on these dimensions.

The objectives of the analysis were: (1) to clarify the conditions under which crew interaction skills might be expected to affect technical performance of crews and (2) to begin the process of describing specific crew behaviors that potentially contribute to better technical performance.

The second aspect of the analysis focused on the usefulness of the BARS rating scales per se in evaluating team interactions skills. We examined crew ratings on the BARS scales to assess (1) whether there was variability in crew scores on the BARS dimensions and (2) whether there was a relationship between BARS ratings of team skill and crew technical performance on the scenarios.

Researchers have had limited success to date in finding a positive link between crew interaction skills and technical performance. While the results we present below are by no means definitive, they provide evidence suggestive of a positive link between crew interaction skills and technical performance, and point to the kinds of studies and analyses that could provide more definitive results.

\subsection{Cognitively Demanding Situations Where Good Crew Interaction was Important}

We identified three types of cognitively demanding situations where specific types of crew interaction appeared to contribute positively to successful crew performance from a technical perspective. These were:

- Cases where operators needed to pursue multiple objectives. Specifically, casés where they had to manage dual requirements to (1) proceed through the EOPs to cool down the plant and bring it to a more stable state in a timely manner and (2) engage in extra-procedural activities to handle aspects of the situation that were not covered by the EOPs;

- Cases where situation assessment required integration of information that was distributed across crew members; and

- Cases where crews had to evaluate the appropriateness of a procedure path and/or decide whether to take actions not explicitly specified in the procedures.

In each case we examined characteristics of crew interaction that appeared to contribute positively to crew performance from a technical perspective.

\subsubsection{Cases Where Crews Needed to Pursue Multiple Objectives}

In the two ISLOCA scenarios crews needed to engage in extra-procedural activity to identify and isolate the leak into the RHR. They also needed to proceed with the cooldown as rapidly as possible to reduce the effect of the leak and stabilize the plant. We examined how crews organized themselves to deal 
with these multiple objectives, and whether some crew styles of organization led to better performance than others. These behaviors fall under the BARS "adaptability" dimension of crew interaction skills.

Two crew styles of organization were identified. Some crews appeared to alternate between following the steps in the EOP and situation assessment and response planning activities. For example, when these crews got to the step in the LOCA procedure requiring them to identify and isolate the leak, they tended to stay a long time on that step. This crew style was labeled "alternate." A second crew style we identified was characterized by a tendency for the crew to divide into two subgroups, with one subgroup concentrating on trying to identify and isolate the ISLOCA and the second subgroup concentrating on moving through the procedures in order to get to the cooldown more quickly. For example, in the case of one crew (Crew F) the SO explicitly requested that the $\mathrm{SS}$ and $\mathrm{RO}$ use the ISLOCA procedure to try and identify and isolate the leak into the RHR, while he and the BOP continued with the LOCA procedure. We labeled this crew style "divide and conquer."

We examined whether one crew style of organization enabled the crews to reach a cooldown state more quickly than the other. We computed the time in minutes from reactor trip to the time the crews started the Post-LOCA Cooldown and Depressurization procedure. ${ }^{43}$ In both the case of ISLOCA 1 and ISLOCA 2 the crews that were identified as "divide and conquer" reached the cooldown procedure faster than the crews that were identified as "alternate."

In ISLOCA 1 seven crews reached the cooldown procedure. Of these, four crews were classified as "divide and conquer" and had a mean time of 34 minutes to get to the cooldown procedure. Three were classified as "alternate" and had a mean time of $\mathbf{4 2}$ minutes to get to the cooldown procedure. In the case of ISLOCA 2 two crews were classified as "divide and conquer" and had a mean time of 32 minutes to reach the cooldown procedure. Seven were classified as "alternate" and had a mean time of 56 minutes to get

43 In the case of the two crews (Crew 6 and Crew 4) that transitioned to the ISLOCA procedure, the time to cooldown was computed as the time from reactor trip to the Loss of Emergency Coolant Recirculation procedure. to the cooldown procedure. Collapsing across the two scenarios the mean time to cooldown for "divide and conquer" crews was 33 minutes $(n=6)$, while the mean time to cooldown for "alternate" crews was 52 minutes $(n=10)$. This difference is statistically significant using a two-tailed t-test $(p<0.05)$.

Since proceeding expeditiously to the Post-LOCA Cooldown and Depressurization procedure is a high priority goal, the results suggest that a "divide and conquer" crew organization style may have certain benefits over an "alternate" crew style because it is likely to allow the crews to proceed through the EOPs more rapidly. These benefits only hold if the two s".h groups maintain close communication and ccudination to ensure that they are not taking actions that interfere with one another. The groups that used a "divide and conquer" strategy tended to use the SO as a focal point and alerted him of all major actions before taking them. Crew 7 in ISLOCA 2 provides an example of a case where the actions taken by the subgroup that was pursuing the source of the leak into the RHR (isolating the CCW service loop) affected activities of the subgroup that was working through the Post-LOCA Cooldown and Depressurization procedure (procedure steps that assumed the $\mathrm{CCW}$ service loop was available). Because the two subgroups communicated their actions, a potential impasse was identified and resolved.

These results point to the importance of the team skills of communication, coordination, and adaptability to changing plant conditions in dealing with situations that require simultaneous pursuit of multiple objectives. More specifically, the results suggest particular crew behaviors that may lead to improved technical performance (i.e., crews breaking up into subgroups with the $\mathrm{SO}$ as the point of focus for communication and coordination).

\subsubsection{Cases Where Situation Assessment Required Integration of Information Across Multiple Crew Members}

A second case where crew interaction skills appeared to be important to technical performance was in forming correct situation assessment in cases where the pieces of evidence that had to be identified and integrated were distributed across crew members. 
Two of the BARS dimensions of crew interaction skills appeared to be important to technical crew performance in these cases. One was communication. In the simulated scenarios cases arose where a piece of evidence that was needed to identify the plant fault was only seen by a single crew member, and there was no EOP step that specifically requested that piece of information. In those cases correct situation assessment depended on the crew member recognizing the value of the information and communicatin 3 it to the rest of the crew. A specific case in point was the rupture of the PRT in ISLOCAs 1 and 2 . The crew member who noticed the symptoms in the PRT needed to communicate that information to the other crew members in order for the leak into the RHR to be identified. In one case (Crew 3, ISLOCA 2) one of the crew members knew the PRT had ruptured but failed to communicate it to the SO and the rest of the crew. This crew did not identify the problem in the RHR until late in the event.

A second dimension of crew interaction skill that appeared to be important for correct situation assessment was openness. The results showed that crew members in all positions contributed positively to hypothesis generation and revision. This was shown most clearly in the case of ISLOCA 1 . While the first hypothesis generated to explain the plant symptoms was most often generated by the SO (five out of 11 cases), there were also cases where it was the SS or the BOP that generated the first hypothesis. Further, when we looked at cases where the initial hypothesis was revised, and examined which crew member suggested the revised hypothesis, we found that crew members in all positions were represented (i.e., RO, STA, SS, BOP). In cases where the first hypothesis that was generated was relatively implausible, and it was revised to a more plausible explanation, the crew member who suggested the revised hypothesis was different from the crew member who suggested the original hypothesis. These results suggest that having multiple crew members participate in the generation and revision of hypotheses contributes positively to correct situation assessment. In turn, this suggests that "openness" of crew members with respect to suggesting and critiquing hypotheses contributes positively to correct situation assessment.

\subsubsection{Cases Where Crews Had to Evaluate Whether to Take Actions Outside the Procedures}

A third type of situation where a positive role of crew interaction on technical performance was identified was when crews had to evaluate the appropriateness of a procedure path and/or decide whether to take actions not explicitly specified in the procedures. Analysis indicated that "openness" in crew interaction was important both from the perspective of generating proposed actions to take, and from the perspective of evaluating those proposed actions. A clear example occurred in ISLOCA 1 where crews considered whether to isolate the affected RHR train. Examination of crew performance in that case revealed that the initial suggestion to isolate the RHR was made by crew members in a variety of positions (i.e., RO, STA, SS, BOP, SO). In all cases the crews did decide to isolate the RHR train but only after examination of the possible consequences of the action by the crew as a whole. The final decision was made by the $\mathrm{SO}$ after soliciting input from other $\mathrm{crew}$ members and approval from the SS. Similar results were observed in the LHS 2 scenario where crews had to decide whether to deviate from the literal requirements of procedure steps in the Reactor Trip Response procedure.

\subsubsection{Summary}

The analysis provided above revealed cognitively demanding situations where contributions of multiple crew members appeared to play a role in successful crew technical performance. It also suggested some specific crew behaviors (e.g., dividing into subteams; communicating indications of abnormal plant behavior; volunteering hypotheses; critiquing hypotheses; proposing response actions; evaluating proposed actions) that fell under the BARS dimensions of crew interaction skills that appeared to contribute positively to the technical performance of the crews.

Section 4.2 contains the results of the crew ratings on the BARS scales, providing a further description of specific crew behaviors that characterized good crew performance on the BARS scales, and appeared to contribute positively to technical performance of the crews. 
Crew Interaction

Implications of these results for the training and testing of crew interaction skills are discussed below following presentation of the BARS ratings results.

\subsection{BARS Ratings of Crew Interaction Skills}

The BARS scales were used to rate crew performance in each of the scenarios. The resulting $B A R S$ ratings were examined to assess (1) whether there was variability in crew scores on the BARS dimensions and (2) whether there was a relationship between BARS ratings of team skill and crew technical performance on the scenarios.

\subsubsection{Variability among Crews on BARS Dimensions}

Table 4.1 presents the mean ratings of the crews on each of the BARS dimensions for each scenario. The standard deviations appear in parentheses. There was variability in crew ratings on four of the six dimensions. Little or no variability was observed in the ratings of Team Spirit and Task Focus.

Crews varied extensively in degree of communication. Specific behaviors that contributed to a high score on the communication dimension included making sure that all important plant changes and crew actions were known to all crew members, providing periodic summaries of current situation assessment, and announcing activities that were about to be started that would strongly affect plant state (e.g., depressurizing a steam generator that would result in a cooldown). Cases where crews failed to communicate critical plant state information (e.g., that the PRT ruptured) or operator actions (e.g., closing the PORV block valve) resulted in lower scores on the communication dimension.

Crews varied in the 'openness' dimensions. Crews with a high openness score tended to include crew members who volunteered situation assessments or suggestions for actions, and SOs who explicitly solicited the opinion of crew members and sought consensus for all major situation assessments and decisions.

Crews also varied on the dimension of Task Coordination. There were several opportunities to observe the role of crew coordination. In the ISLOCA scenarios crews differed in how they organized themselves to deal with both the need to identify and isolate the leak outside containment and the need to proceed expeditiously to the Post-LOCA Cooldown.

In the Loss of Heat Sink scenarios crew coordination was required to depressurize the RCS and block the SI signal without inadvertently safety injecting. Crews that scored high on the coordination dimension tended to have SOs that provided the operators an overview of the steps about to be taken. These SOs tended to give the crew an overview of the whole maneuver before initiating the RCS depressurization and to explicitly assign specific roles for the different operators.

Table 4.1 Mean ratings of crews on the BARS dimensions. (Standard deviations appear in parentheses.)

\begin{tabular}{l|l|l|l|l|l|l|} 
Scenario & Communic. & Openness & Coordination & Team Spirit & \multicolumn{1}{|c|}{$\begin{array}{c}\text { Task } \\
\text { Focus }\end{array}$} & Adapt. \\
\hline ISLOCA 1 & $5.1(1.0)$ & $5.5(0.8)$ & $5.0(1.4)$ & $4.2(0.4)$ & $4.0(0)$ & $4.6(1.7)$ \\
ISLOCA 2 & $4.5(1.5)$ & $5.2(1.5)$ & $4.8(1.4)$ & $3.7(1.0)$ & $4.0(0)$ & $5.3(1.2)$ \\
LHS 1 & $4.6(1.1)$ & $5.0(0.9)$ & $4.3(1.5)$ & $3.9(0.4)$ & $4.0(0)$ & $4.8(1.6)$ \\
LHS 2 & $4.9(0.9)$ & $5.3(0.5)$ & $5.1(1.2)$ & $4.0(0)$ & $4.0(0)$ & $5.1(1.2)$ \\
\hline
\end{tabular}


The following protocol segment provides an example of an SO who looked ahead in the procedures and provided the operators an overview of maneuvers to come.

\section{Looking Ahead in the Procedures Crew 1}

This protocol segment starts just at the point where the crew reaches the RCS depressurization step.

14:48:29 SO reads caution prior to step 7.

14:48:47 Step 7

14:49:05 SO to BOP: Be alert for monitoring pressure for RO, so that when we get to less than 1920, RO can block steam line pressure SI, low pressurizer pressure. SI.

14:50:11 SO: Alright, two things here guys. One, we know that we can do Charlie feed reg. valve if we need to. Don't worry about that. Don't forget that we have the ability of opening it. Two, we want to go to arm so that we don't lose the ability to have the block valve open on the pressurizer PORV that you are using. Team work here. (To RO:) You open the PORV; when pressure gets less than 1920 you close the PORV. (To BOP:) At that time you're over here blocking low pressure SI and low steam line pressure SI. Both of you ready. Proceed.

Crews also varied on the dimension of 'adaptability.' The 'adaptability' dimension was used to rate crews on how quickly they detected and responded to changing plant circumstances. High ratings on this dimension tended to be given to crews that detected and pursued the primary symptoms in each event while continuing to proceed through the EOPs. In the ISLOCA these were the symptoms of a leak outside containment. In the Loss of Heat Sink scenario the primary symptoms were those of a leaking pressurizer PORV.

The dimensions of 'team spirit' and 'maintaining task focus in transitions' seemed less useful in that there seemed to be less variance across crews on these dimensions. All the crews showed positive team spirit. Expressions of anger or frustration at each other were extremely rare.

The fact that variability in ratings occurred across crews on four of the six dimensions suggests that these dimensions may be useful in evaluating crew interaction performance. Previous attempts to use the BARS scales had found limited variability in crew ratings on the events examined. It is possible that there was more variability in crew interaction performance in this study because of the greater cognitive demands of the scenarios. As discussed in Section 3, a number of cognitively demanding situations arose in those scenarios where good technical performance depended on the contributions and coordination of multiple crew members. It is possible that these scenarios placed greater demands on team interaction skills and thus provided the opportunity to observe variability in performance.

\subsubsection{Evidence of a Link between Crew Interaction Skills and Technical Performance}

We also examined whether a link could be established between crew performance on the BARS ratings of crew interaction and crew technical performance. In general, crew technical performance on the scenarios was very good. The large majority of crews correctly identified the leaks and took appropriate action in attempting to isolate the leaks. Nevertheless, in each scenario there was one crew whose technical performance was clearly less good than that of the other crews (Crew L in ISLOCA 1, Crew 3 in ISLOCA 2, Crew H in LHS 1, and Crew 11 in LHS 2.) These four crews failed to reach a correct situation assessment and as a result failed to take actions needed to isolate the leaks.

BARS ratings for these four crews on the events in question were compared to the BARS ratings for the remaining cases ( 33 cases). The mean ratings on the four BARS scales for which variability across crews was observed are presented in Table 4.2. Crews that 
Crew Interaction

Table 4.2 Mean BARS ratings for crews that differed in technical performance. (Standard deviations appear in parentheses.)

\begin{tabular}{cccccc}
\hline $\begin{array}{c}\text { Crew Technical } \\
\text { Performance }\end{array}$ & $\begin{array}{c}\text { Number of } \\
\text { Crews }\end{array}$ & Communic. & Openness & Coordination & Adapt. \\
\hline Good & 33 & $4.9(0.9)$ & $5.4(0.8)$ & $5.0(1.3)$ & $5.2(1.3)$ \\
Less Good & 4 & $3.5(2.1)$ & $4.5(1.9)$ & $3.5(1.3)$ & $3.0(0.8)$ \\
\hline
\end{tabular}

were classified as 'good' from a technical perspective had higher mean BARS ratings on all four BARS dimensions than the crews that were classified as 'less good' from a technical perspective. Analyses of variance indicated that the mean differences in BARS ratings were statistically significant $(p<0.05)$ in the case of three of the four BARS dimensions: communication, coordination, and adaptation. In the case of the dimension of "openness" the mean difference in ratings was not statistically significant.

The statistically significant difference that was obtained on some of the BARS dimensions between crews that performed technically well on the scenarios and crews that performed less well is an important finding. Researchers have generally had difficulty establishing a link between team interaction skills and technical performance. If the finding is reliable it would support the position that team interaction skills contribute to better technical performance.

However, because only a single rater (the first author) was used, the reliability of the BARS ratings obtained, and therefore the robustness of the evidence connecting BARS ratings to technical performance, is not clear. Because of the potential importance of the result it may be worthwhile to attempt to replicate the result using a larger group of raters.

\subsection{General Discussion of Team Interaction Skills Results}

The results served to clarify conditions under which crew interaction skills may be expected to affect technical performance of crews. They also revealed specific crew behaviors that may characterize good crew interaction and contribute to technical performance. In addition, they provided evidence of a link between crew interaction skills and technical performance. While the results are not definitive, they point to the kinds of studies and analyses that could provide more definitive results.

There was more variability in BARS ratings of crew interaction skills in this study than in previous studies (Montgomery et al., 1992). One possible explanation is that the scenarios used in the present study were more cognitively demanding. A number of cognitively demanding situations arose in these scenarios where better technical performance depended on the contributions and coordination of multiple crew members. These scenarios may have placed greater demands on team interaction skills and thus provided the opportunity to observe variability in performance.

This argument suggests that future studies that attempt to establish a link between team interaction skills and technical performance should employ scenarios that are specifically designed to be demanding from the perspective of team interaction. The scenarios should be designed so that technical performance depends on the contributions and coordination of multiple crew members. The results presented in Section 4.1 begin to point to the kinds of cognitively demanding situations where crew interaction skills would be expected to affect crew technical performance. Specific crew behaviors that are indicators of good crew interaction should be identified a priori. The analysis should focus on whether crews exhibit these behaviors, and in turn whether these behaviors are linked to good technical performance. Researchers examining aircrew team interaction skills have proposed similar methodological approaches (Fowlkes, Lane, Salas, Oser and Pince, 1992).

The results also pointed to specific crew behaviors that appeared to characterize better performance on 
the BARS dimensions of crew interaction skills and to contribute positively to technical performance of the crews. Examples include splitting into subteams, having all crew members participate in situation assessment and response planning activities, ensuring that all crew members are cognizant of key plant state information and control actions that are taken, and providing periodic recaps of current situation assessment and upcoming activities.
Understanding the specific behaviors that characterize team skills is important for guiding development of team skills training programs. While the present results are suggestive, more research is needed to establish a definitive link between specific crew interaction behaviors and crew technical performance. 


\section{Discussion of Results and Their Implications}

\subsection{General Discussion}

In Section 1 we contrasted two alternative views of the nature and extent of cognitive activity required of operators to adequately handle emergencies. One view was that in emergencies the operator's primary role is to follow the EOPs by rote. According to this view all that is needed of operators is that they be able to understand and follow the individual steps in the EOPs.

This position was contrasted with the view that situation assessment and response planning continue to be important for successful operator performance, even when EOPs are employed. According to this view situation assessment and response planning enable crews to identify and deal with situations that are not fully addressed by the procedures.

The results of this study provide support for the second position. We found a number of situations that were not fully addressed by the EOPs. These included:

- An EOP step that explicitly requested that crews identify and isolate a leak on their own (ISLOCA 1);

- A case where the procedure containing relevant guidance could not be reached within the EOP transition network (ISLOCA 2);

- Cases where operators needed to determine whether plant behavior was the result of known manual and/or automatic actions (e.g., a controlled cooldown) or the result of a plant fault (all four simulated events);

- A case where operators were required to evaluate the appropriateness of procedure steps given the specifics of the situation (LHS 2);

- Cases where operators had to evaluate the appropriateness of a procedure path and take action to redirect the procedure path (ISLOCA 2; LHS 1; LHS 2);
- A case where operators had to decide whether to manually initiate a safety system based on consideration and balancing of multiple goals related to safety (LHS 1).

In all these cases we found evidence of operators actively engaging in situation assessment and response planning in handling the situation.

There are three alternative interpretations of these results, each with distinct implications. If one starts from the premise that procedures should provide detailed guidance for every contingency, then one interpretation of the results is that they demonstrated deficiencies in the particular procedures that were included in the study. According to this view if situations are identified that are not covered by the procedures, then the procedures should be rewritten to handle those situations. Given this view, the results have primary implications for the specific procedures employed in the study.

A second view is that the EOPs are not intended to diagnose and respond to particular faults optimally. They are intended to provide a systematic approach to emergency response that minimizes the possibility of core damage. According to this view, while the operators may have engaged in situation assessment and response planning in these scenarios, these cognitive activities were not necessary, and were possibly not even desirable. Had the operators followed the procedures implicitly they would have eventually been directed to take actions that would have mitigated the consequences of the leaks and prevented core damage. Given this view, the primary contribution of the study is that it demonstrates that operators take a more active role in diagnosing and responding to events than might have been believed; however, the results have minimal implications for training and procedures.

A third view is that the types of situations that were identified in the study are generic classes that are likely to arise in other emergency scenarios.

According to this view, the complexity of NPPs make it difficult to anticipate and develop EOPs that cover 
every possible contingency in detail; therefore it is reasonable to assume that situations may arise that are not fully addressed by the procedures. It will be important in such situations for the operators to have the ability to form accurate situation assessments and to generate response plans to cover aspects of the situation that are not fully addressed by the procedures. Examination of recent actual incidents support this position (NRC, NUREG-1455; Kauffman et al., 1992).

A logical consequence of this third view is that in the development and evaluation of training and control room aids (e.g., procedures, displays, decision-aids), explicit attention should be paid to supporting operator situation assessment and response planning.

The results of the study by themselves do not definitively support one view over the others. However, we present evidence from actual incidents, experiences in other domains, and logical arguments, in support of the third view.

In Section 5.2 we summarize some of the main results of the study that provided evidence of a need for situation assessment and response planning on the part of the crews. In Section 5.3 we describe the roles that situation assessment and response planning played in guiding operator performance in these cognitively complex emergencies. In Section 5.4 we examine the three alternative ways of interpreting the results and their implications. We provide arguments in support of the third view that operators need to engage in situation assessment and response planning in handling emergencies. In Section 5.5 we discuss the implications of this view for the development and evaluation of training and control room aids, as well as for Human Reliability Analyses.

We conclude with some specific comments on the value of simulator-based empirical studies of operator performance.

\subsection{Summary of Results}

Situations arose in each of the scenarios where operators needed to engage in situation assessment and response planning.
In ISLOCA 1 the crews were required to identify and isolate the leak into the RHR without explicit procedural guidance. In ISLOCA 2, while there was a procedure transition available to an ISLOCA procedure, in the case of several of the crews it could not be reached. Even in the cases where the ISLOCA procedure was reached, it did not cover all aspects of the situation (i.e., the leak into the $\mathrm{CCW}$ ).

Most crews actively sought information to help identify the sources of leaks into the RHR and CCW, and identified and took actions in attempting to isolate the leaks. They actively utilized resources beyond the EOPs to support their identification and isolation of the leaks. Without active situation assessment, knowledge-driven monitoring, and response planning, they would not have been able to identify and isolate the leaks.

At the same time, most of the crews recognized the importance of continuing to proceed through the EOPs. They perceived getting to the Cooldown and Depressurization procedure as a high priority activity. Balancing the dual requirements to pursue the leak into the RHR with the need to proceed expeditiously through the EOPs provided one of the most challenging aspects of the ISLOCA scenarios.

The ISLOCA scenarios also provided evidence of crews actively engaged in reasoning about the procedure logic. Protocol segments showed crews reasoning at two levels. They were engaging in situation assessment and goal identification. At the same time they were reasoning about the strategies underlying the EOPs, and the EOP transition network logic to assess whether the procedure path they were on would enable them to achieve plant goals in a timely manner.

We found instances where monitoring the appropriateness of the procedure path enabled crews to identify when they were in an unproductive loop, and to identify another procedure path that would allow them to take necessary actions more expeditiously.

The Loss of Heat Sink scenarios provided further evidence that complex multiple fault conditions can arise that require operators to actively engage in situation assessment and response evaluation. In the Loss of Heat Sink scenarios the procedures provided no guidance in identifying and responding to the 
leaking pressurizer PORV. The majority of crews were successfully able to detect the symptoms on the primary system and integrate them to identify the steam space leak. This difficult cognitive task required recognizing that the primary side behavior could not be entirely explained by the ongoing cooldown. This task required qualitative reasoning about the size and direction of effects on the primary system that could be expected from the rapid depressurization of the steam generators.

In the Loss of Heat Sink 1 scenario, the crews were faced with a decision regarding manual initiation of a safety system. The only EOP guidance available to them was in a caution that indicated that they had discretion to turn on the safety system if conditions in the plant "degraded." The decision of whether to turn on the safety system required balancing multiple goals. Manual initiation of the safety system would respond effectively to the degrading conditions in the primary system due to the leaking PORV, but could potentially delay recovery of heat sink. The crews had difficulty with this aspect of the scenario. Most of the crews did not recognize that they had the discretion to decide whether to turn on the safety system. Further, few of the crews showed evidence of considering the tradeoffs involved. The majority of crews chose to let conditions continue to degrade until a criterion was reached for which more explicit procedural guidance was available.

Loss of Heat Sink 2 provided additional opportunity to examine the role of situation assessment and response planning in guiding crew performance. In this event crews returned to the Reactor Trip procedure after recovering feedwater using the condensate system. In this case the crews were explicitly required, based on training and EOP background documents, to use their judgment in deciding the appropriateness of particular procedure steps. Most of the crews correctly recognized that some of the steps were inappropriate to the situation and should not be followed. This included steps that called for initiation of a safety system. The decision that initiation of the safety system was not needed was based on situation assessment. The crews had to determine that the conditions in the primary system were due to cooldown and not a plant fault. This was not a simple decision, as attested by the fact that, in the case of two of the crews who faced that decision, there was a leak present (leaking pressurizer PORV), but the crews nevertheless initially attributed the primary side symptoms to cooldown and decided against manual initiation of SI.

In general, across scenarios, the majority of crews performed well. They identified the faults and took appropriate action in response. The behavior of these crews clearly indicated that they were actively engaged in situation assessment and response planning.

While most of the crews performed well, variability in performance was observed. Crews differed in the extent to which they detected plant symptoms, actively sought an explanation for unexpected findings, and attempted to come up with a coherent explanation that accounted for all the observed symptoms. In each scenario there was at least one crew that failed to identify the source of the problem correctly and consequently failed to take appropriate action to mitigate it. Given that the number of crews that participated in each scenario ranged from eight to eleven, this means that approximately $10 \%$ of the crews experienced difficulty. ${ }^{44}$ The fact that not all crews in the scenarios formed the correct situation assessment suggests that there is room for improvement. Section 5.5 discusses potential ways to enhance crew situation assessment and response planning.

Finally, the results clarified the role of group interaction in situation assessment and response evaluation. The results indicated that multiple crew members contributed evidence and opinion in formulating situation assessments and evaluating response options. This was particularly true when the crews reached points in the EOP where their assessment of the correct action to take diverged from the actions specified in the procedure. At those points the SOs generally sought input from all crew members and approval from the SS before deciding to diverge from a procedure step.

\footnotetext{
${ }^{44}$ While approximately $10 \%$ of the crews failed to reach the correct situation assessment, it should be pointed out that plant safety was always maintained. Even if these crews did not come to the correct situation assessment the EOPs would have eventually led them to take action that would have prevented any serious consequences to core integrity.
} 


\subsection{The Role of Situation Assessment and Response Planning in Cognitively Demanding Emergencies}

Situation assessment and response planning enabled the crews to handle aspects of the scenarios that were not fully covered by the EOPs. This section describes the role that situation assessment and response planning played in guiding operator response in the scenarios. It is reasonable to assume that the results observed can be generalized beyond these particular crews and scenarios, and that situation assessment and response planning would play a similar role in guiding operator performance in other cognitively demanding emergencies.

\subsubsection{Situation Assessment}

The scenarios provided extensive evidence of crews trying to develop an understanding of plant state. We observed operators engaging in knowledge-driven monitoring to confirm their understanding of a situation and seeking explanation for unexpected plant behavior. We also observed operators actively trying to form a coherent explanation to account for multiple symptoms across diverse systems. These activities enabled the crews to identify and respond to problems that were not fully addressed by the EOPs.

Situation assessment enabled the crews to:

- Detect abnormal plant behavior earlier in the event than would be possible if they waited for an alarm or a step in the procedure to check those parameters;

- Detect symptoms or alarms that they had missed earlier;

- Identify and deal with additional problems that were not addressed by the procedures.

It is reasonable to assume that situation assessment would play a similar role in enabling crews to identify and deal with problems in other cognitively demanding situations.
The importance of situation assessment is underscored by the frequency of recent actual incidents where crews were required to discriminate actual malfunctions from failed sensors or false alarms (Kauffman et al., 1992). The results of the present study as well as analyses of actual incidents suggest that it is important for operators to develop and maintain an accurate situation assessment in order to handle aspects of incidents that are not fully addiessed by the procedures. Important elements of situation assessment include (1) an awareness of abnormal plant symptoms, (2) an assessment of the likely malfunctions that could produce those symptoms, and (3) an awareness of manual and automatic system actions that are being taken, and their effect on plant state.

\subsubsection{Response Planning}

The scenarios were designed to produce situations where operators were required to engage in response planning. In some cases this involved identifying and evaluating response actions on their own. In other cases, it involved monitoring the appropriateness of response actions specified in the procedures, and adapting the procedures to the situation if judged necessary.

We found evidence of crews reasoning at two levels. They engaged in situation assessment and goal identification. At the same time they monitored the procedure path they were following to evaluate progress toward high priority goals.

Response planning enabled the crews to:

- Move through the procedures efficiently;

- Catch and recover from errors -- both operator errors and errors in the procedures;

- Assess whether the procedure path they were on was appropriate to the situation;

- Fill in gaps and adapt procedures to the situation; and

- Deal with unanticipated situations that went beyond the available procedural guidance. 
It is reasonable to assume that the role of response planning in enabling crews to deal with these situations would generalize to other cognitively demanding emergencies.

The results provide evidence that it is important for operators to be able to develop and evaluate response plans. It is also important for them to understand the assumptions and logic behind the EOPs. This understanding includes the intent behind specific procedure steps, the overall response strategies inherent in the procedures, and the transition logic among particular procedures in the EOPs.

\subsection{Alternative Views of the Role of Procedures and Implications of Results}

The results provide clear evidence that situations arose in the simulated scenarios where operators needed to engage in situation assessment and response planning to deal with aspects of the event that were not fully addressed by the EOPs.

At the beginning of Section 5 three alternative interpretations of these results were outlined that have different implications for training, procedures, and decision aids. In this section these three views are examined in more detail. While the results of the study do not definitively support one view over the others, arguments are presented in favor of the third view: operators need to engage in situation assessment and response planning to handle unanticipated situations that are not fully covered by the EOPs. This view has implications for training, procedures, and decision aids.

\subsubsection{View 1: Procedures Should Provide Detailed Guidance for Every Contingency}

One view starts from the premise that procedures should provide detailed guidance for every contingency. Given this premise, the results could be viewed as providing evidence of deficiencies in the particular procedures that were included in the study. According to this view if situations are identified that are not covered by the procedures then the procedures should be rewritten to provide detailed guidance for those situations.
While this position is viable in principle, in practice it is likely to be difficult to anticipate and provide detailed guidance for every possible contingency. This argument is supported by experience in attempting to develop detailed procedural guidance in other domains (Roth, Bennett, and Woods, 1987; Suchman, 1987). It is also supported by analyses of actual incidents that often involve multiple faults and complications whose possibility had not been foreseen (Kauffman, Lanik, Trager, and Spence, 1992; NRC, NUREG-1455; Perrow, 1984; Wagenaar and Groeneweg, 1987).

Some of the cases identified in the scenarios could be handled by rewriting the particular procedure to explicitly deal with the case. An example is the situation that arose in ISLOCA 1 where the EOPs asked the operators to identify and isolate the leak without providing further guidance. This procedure could be rewritten to provide more detailed guidance with respect to identifying and isolating the leak.

There were other cases, however, that could not be easily handled by rewriting the procedures.

Examples include the case that arose in ISLOCA 2, where detailed guidance for identifying and isolating the ISLOCA was available but could not be reached through the EOP transition network. The reason the ISLOCA procedure could not be reached had to do with the detailed dynamics of the event that determined when symptoms came in relative to when procedure steps were reached. Developing procedures that anticipate and provide for the variety of possible event trajectories that could arise would be a difficult task.

Procedure writers recognize limits in their ability to foresee all possible situations. In some circumstances operators are explicitly directed by the EOPs to take action based on their own situation assessment. There were three cases in the simulated scenarios where the procedures or related background documents explicitly directed operators to determine appropriate action based on their own situation assessment:

1. The case in the ISLOCA scenarios where operators were asked whether pressure in all steam generators is "stable or increasing;"

2. The caution that appeared in the loss of heat sink procedure that provided the operators discretion in initiating a safety system; 
3. The case that arose in LHS 2 where operators were expected to determine whether particular procedure steps in the Reactor Trip procedure were appropriate to the situation and should be followed.

\subsubsection{View 2: Procedures Are Not Intended to be Optimal.}

A second view is that the EOPs are not intended to diagnose and respond to particular faults optimally. They are intended to provide a systematic approach to emergency response that minimizes the possibility of core damage. Had the operators followed the procedures by rote they would have eventually been directed to take action that would have mitigated the consequences of the leaks and prevented core damage. According to this view, while the operators may have engaged in situation assessment and response planning in these scenarios, these cognitive activities were not necessary.

This position underlies the development of the EOPs and provides the rationale for requiring operators to follow procedures by rote. The results of this study do not contradict this position. In both the ISLOCA and the LHS scenarios, had the operators followed the procedures by rote they would have eventually been directed to take action that would have prevented severe core damage; however, conditions would have degraded significantly before the procedures directed the operators to take action to address the problem. 45 This raises a concern because when conditions are allowed to degrade the potential for risk is increased.

\subsubsection{View 3: Situation Assessment and Response Planning Enable Operators to Handle Unanticipated Situations}

A third view is that the complexity of NPPs make it difficult to anticipate and develop EOPs that cover every possible contingency in detail. According to this view it is reasonable to assume that situations may arise that are not fully addressed by the procedures. In such situations the ability of operators

\footnotetext{
${ }^{45}$ In the case of the ISLOCAs a large amount of primary coolant would have been depleted. In the case of LHS 1, reactor vessel level would have decreased below $40 \%$.
}

to form accurate situation assessments and to generate response plans to cover aspects of the situation that are not fully addressed by the procedures will be important.

Several lines of evidence support this position including, experience in developing detailed procedural guidance in other domains (Roth, Bennett, and Woods, 1987; Suchman, 1987); experience in introducing automation (Norman, 1986); and analyses of actual incidents that involved multiple faults and complications that had not been foreseen (Kauffman, Lanik, Trager, and Spence, 1992; NRC, NUREG-1455; Perrow, 1984; Wagenaar and Groeneweg, 1987.)

The results of the study, taken in combination with evidence from actual incidents, and experiences in related domains support the position that situation assessment and response planning enable operators to handle unanticipated situations that are not fully addressed by procedures. In Section 5.5 we discuss the implications of this view for the development and evaluation of training and control room aids, as well as for human reliability analyses.

\subsection{Implications of Results}

The view that unanticipated situations may arise in actual incidents where operators need to engage in situation assessment and response planning to deal with aspects of the situation that are not fully addressed by the procedures has potential implications for:

- Training of operators;

- Development of displays and decision-aids to support operator cognitive performance; and

- Human reliability analysis.

\subsubsection{Implications for Training}

The view that situations may arise where crews need to engage in situation assessment and response planning suggests that in developing and evaluating operator training programs attention may need to be paid to the development of these cognitive skills. 


\section{Kinds of Operator Knowledge}

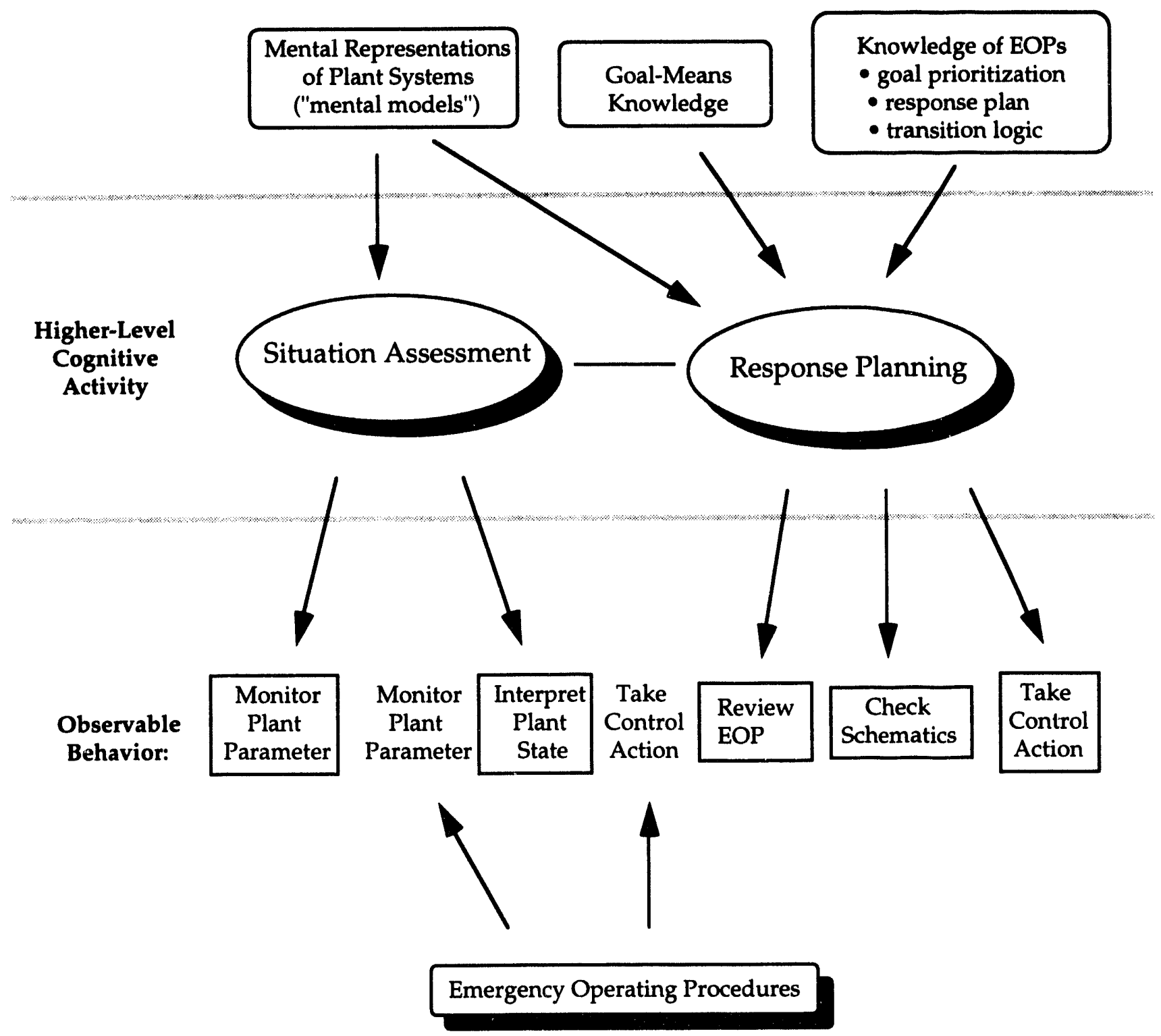

Figure 5.1 Operator knowledge required to support situation assessment and response planning.

While most of the crews in the study were able to identify the leaks correctly and take appropriate action, not all the crews formed an accurate situation assessment. Crew performance might be improved by providing explicit training in situation assessment and response planning.
Figure 5.1 shows three kinds of operator knowledge required to support situation assessment and response planning:

1. Operators need accurate mental models of plant systems. In our study we found evidence of 
situations where crews needed to utilize mental models of physical plant systems and to reason qualitatively about expected effects of different factors influencing plant state in order to localize plant faults and identify actions to mitigate them.

2. Another type of knowledge needed is knowledge of important plant goals and means to achieve them. Our study found evidence that operators needed to reason about plant goals, and evaluate alternative means to achieving them, particularly in the Loss of Heat Sink 1 event.

3. Finally, operators need knowledge of the EOPs, which includes not only knowledge of how to follow the individual EOP steps, but also knowledge of the logic that underlies the EOPs. This includes knowledge of the goal prioritization inherent in the EOPs, knowledge of the response plans embodied in the EOPs and their rationale, and knowledge of the EOP transition network. It may be beneficial to explicitly address these types of knowledge in training programs.

Mumaw, Swatzler, Roth and Thomas (1994) provide a detailed review of training techniques for developing these types of knowledge and cognitive skills.

One way to foster situation assessment and response planning skills is to develop cognitively demanding training scenarios that provide the opportunity to practice specific cognitive skills (Roth, Mumaw \& Pople, 1992). For example, training scenarios can be developed that specifically focus on the ability to form accurate situation assessments. An example is a scenario that requires crews to discriminate effects due to cooldown from effects due to actual malfunctions. Other scenarios can be developed that focus on response evaluation. For example, scenarios can be developed that require operators to evaluate the appropriateness of particular procedure steps to a given situation and to take discretionary action as appropriate.

The objective of the cognitive training would be to build operator skill in handling cognitively demanding events. Since actual incidents typically involve multiple factors that make them unique, cognitive training may better equip operators to handle these unique features resulting in improved safety.

\subsubsection{Implications for Control Room Aids}

The view that unanticipated situations can arise where operators need to engage in situation assessment and response planning also has implications for the development and evaluation of control room aids. In particular, it suggests potential value for displays and decision-aids that are explicitly intended to support situation assessment and response planning.

The results of the study showed that operators sometimes had to engage in situation assessment activity that required tracking multiple influences on plant state and distinguishing plant behavior due to known influences (e.g., a cooldown) from unexpected plant behavior due to an unidentified fault. These judgments often required integrating evidence across space and time. Displays and decision-aids could be developed to support these situation assessment activities.

Similarly, situations arose where crews had to evaluate responses for potential negative consequences. This evaluation step occurred in the ISLOCA incident where crews needed to consider the implications of isolating systems for future recovery activities. It also uccurred in the Loss of Heat Sink event where crews had to consider the positive and negative consequences of initiating SI. Displays and decision-aids that facilitate identification of side efrects and consequences of contemplated actions could be developed to support response evaluation.

The results also have implications for procedures. Two findings in the study have potential implications for design of procedures, particularly computerized procedures. One finding is that it was important for operators to understand the logic and rationale behind the procedures. This has implications for the content and organization of procedures. Another finding is that operators did not necessarily move linearly through a single procedure path. Crews looked ahead in the procedures, they moved back to earlier steps, and they looked at other procedures in parallel as guidance. This finding has implications for the design of computerized procedures. It suggests that ease of navigation through the procedure network is likely to be important for facilitating performance in complex emergencies. 


\subsubsection{Implications for HRA}

The view that operator performance is partly guided by situation assessment and response planning has potential implications for human reliability analyses (HRA). The results indicated that operators are engaged in a number of activities in addition to following the steps in the EOP. Moreover, the results showed that following the EOP steps was not always straightforward. In some cases determination of how to respond to a procedure step depended on situation assessment These results suggest that analyses that focus on the ability of crews to follow individual steps in the EOPs may be insufficient.

The results highlighted the importance of the dynamics of the event in determining what evidence is likely to be available at different points in the event, and what procedure transitions are likely to be made as a consequence. These results suggest that the dynamics of an event play an important role in determining human reliability. An implication is that human reliability assessments are likely to be more accurate if the dynamics of the event are explicitly considered in performing them. This can best be accomplished by running several crews through the specific events using a high fidelity dynamic simulator.

A second implication of the results is that more accurate human reliability assessments are likely to be obtained if analysts take explicit consideration of factors in the events that may complicate situation assessment or response planning. We have developed a 'cognitive demands checklist' that lists many of these factors that can be used to support human reliability assessment. Appendix D contains the 'cognitive demands checklist.'

A third potential implication of the results relates to the estimation of human error probabilities. An HRA analyst who needs to estimate the human error probability for failure to diagnose a rare, complex event, that is not practiced in training, and whose solution is not prescribed in a straightforward way by EOPs, might consider that these types of events were simulated in this study, and that approximately $10 \%$ of the crews did not reach a fully adequate situation assessment. ${ }^{46}$

46 It should be noted that in these scenarios a correct situation assessment was not necessary to take appropriate recovery action.

\subsection{Conclusions}

Among the main conclusions of the study is that, while symptom-based EOPs have greatly reduced the need for operators to develop diagnostic and response strategies on their own in real time, they have not entirely eliminated the need for operators to engage in situation assessment and response planning. In our scenarios a number of cognitively demanding situations arose where operators were required to exercise judgment and take action based on their own assessment of the situation.

The types of situations we identified are generic classes that are likely to arise in other emergency scenarios. The ability of operators to form accurate situation assessments and to generate response plans that adequately address the situation were shown to be important for these situations.

The results are consistent with the view that situation assessment and response planning enable operators to handle unanticipated situations that are not fully addressed by procedures. This view has implications for the development and evaluation of training, and control room aids (e.g., procedures, displays, decision-aids); specifically it suggests that attention should be paid to the need to support and augment operator situation assessment and response planning activities.

The results also have potential implications for human reliability analyses. They suggest that analyses that focus only on the atility of crews to follow individual steps in the EOPs may be insufficient. Human reliability assessments are likely to be more accurate if the dynamics of the event, and the factors that are likely to complicate situation assessment and response planning, are explicitly considered.

A final conclusion of the study regards the value of empirical studies of operator performance in simulated emergencies for addressing human performance issues of concern to the NRC. Well designed empirical studies can provide specific, clear

The EOPs provided recovery paths that did not depend on accurate situation assessment. As a result the probability of failing to take a recovery action would be significantly lower than $10 \%$. 
conclusions for practical decision making. The present study illustrates how empirical studies of operator performance in simulated emergencies can be used to investigate a human performance issue - in this case the role of higher-level cognitive activity in operator response to cognitively demanding emergencies. The study provided: (1) evidence that situations can arise where higher-level cognitive activity on the part of operators is needed and (2) objective data on how different operator crews responded to these situations. 


\section{References}

Barsalou, L. W., Cognitive Psychology: An Overview for Cognitive Scientists, Lawrence Erlbaum Assoc., Hillsdale, N. J., 1992.

Bartlett, F. C., Remembering: A Study in Experimental and Social Psychology, Cambridge University Press, London, 1932.

Beare, A. N., C. D. Gaddy, G. W. Parry, and A. Singh, "An Approach for Assessment of the Reliability of Cognitive Response for Nuclear Power Plant Operating Crews," in Probabilistic Safety Assessment and Management, G. Apostolakis (Ed.), Elsevier Science Publishing Co., Inc., 1991.

Bransford, J. D., Human Cognition: Learning, Understanding and Remembering, Wadsworth Publishing Company, Belmont, Ca., 1979.

Corker, K. M., "An Architecture and Models for Cognitive Engineering Analysis of Advanced Automation Control Environment," Proceedings of the American Nuclear Society Topical Meeting on Nuclear Plant Instrumentation, Control, and ManMachine Interface Technologies, 1993.

Endsley, M. R. "Situation Awareness in Dynamic Human Decision Making: Measurement", Proceedings of the 1st International Conference on Situation Awareness ir. Complex Systems, 1993.

Fowlkes, J. E., N. E. Lane, R. Salas, R. Oser and C. Prince, "TARGETS for Aircrew Coordination Training," Proceedings of the 14th Interservice/Industry Training Systems Conference, 1992.

Fujita, Y., I. Yanagisawa, K. Nakata, J. Itoh, N. Yamane, and R. Kubota, "Modeling Operator with Task Analysis in Mind," Proceedings of the American Nuclear Society Topical Meeting on Nuclear Plant Instrumentation, Control, and ManMachine Interface Technologies, 1993.

Hutchins, E., "The Technology of Team Navigation," in J. Galegher, R. Kraut, and C. Egido, eds., Intellectual Teamwork: Social and Technical Bases of
Collaborative Work, Lawrence Erlbaum, Inc., Hillsdale, NJ, 1990.

Hutchins, E., How a Cockpit Remembers Its Speed, Technical Report, Distributed Cognition Laboratory, University of California at San Diego, 1991.

Kahneman, D., P. Slovic, and A. Tversky, eds., Judgment under Uncertainty: Heuristics and Biases, New York: Cambridge University Press, 1982.

Kauffman, J. V., G. F. Lanik, E. A. Trager, and R. A. Spence, Operating Experience Feedback Report Human Performance in Operating Events, NUREG-1275, Office for Analysis and Evaluation of Operational Data, U.S. Nuclear Regulatory Commission, Washington, D. C., December, 1992.

Klein, G. A. and R. Calderwood, "Decision Models: Some Lessons from the Field," IEEE Transactions On Systems, Man, and Cybernetics, 21, 1018-1026, 1991.

Laird, J. E., A. Newell, and P. S. Rosenbloom, "Soar: An Architecture for General Intelligence," Artificial Intelligence, 33, 1-64, 1987.

Montgomery, J. C., C. D. Gaddy, R. C. LewisClapper, S. T. Hunt, C. W. Holmes, A. J. Spurgin, J. L. Toquam, and A. Bramwell, "Team Skills Evaluation Criteria for Nuclear Power Plant Control Room Crews," working draft, 1992. (Available in NRC Public Document Room.)

Mumaw, R. J., D. Swatzler, E. M. Roth, and Wm. A. Thomas, Cognitive Skill Training for Decision Making. NUREG/CR-6126, U. S. Nuclear Regulatory Commission, Washington, D. C., June, 1994.

Norman, D. A. "Categorization of Action Slips". Psychological Review, 88, 1-15, 1981. 

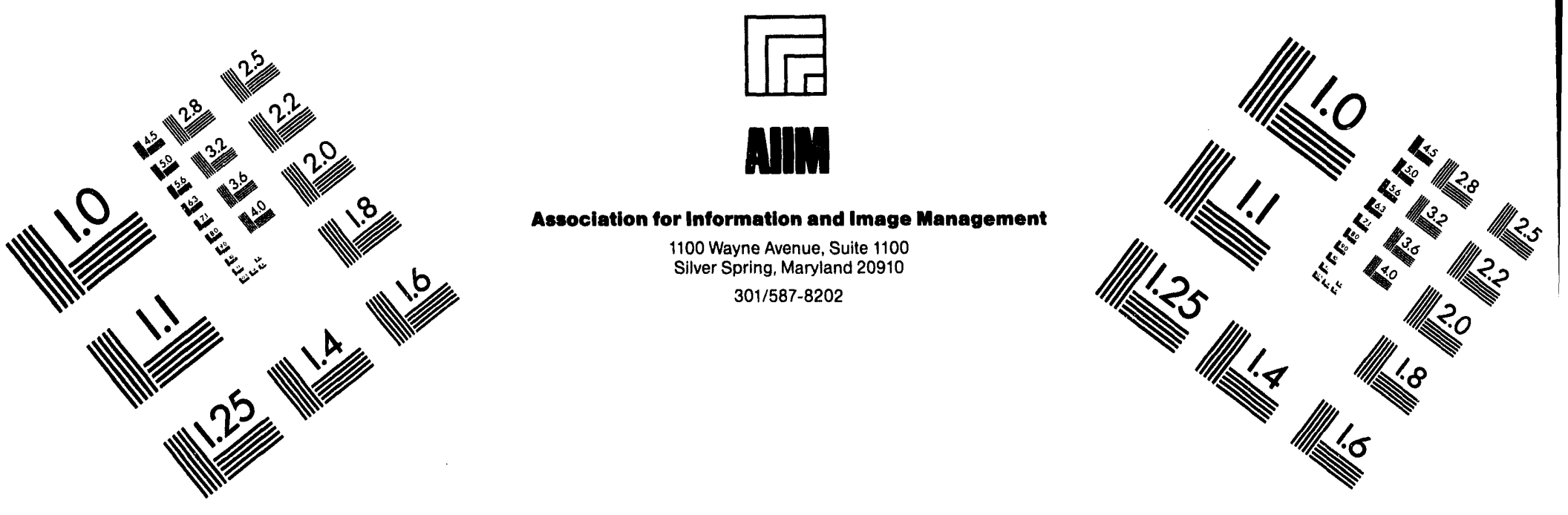

\section{Centimeter}

$\begin{array}{llllllllllllllll}1 & 2 & 3 & 4 & 5 & 6 & 7 & 8 & 9 & 10 & 11 & 12 & 13 & 14 & 15 & \mathrm{~mm}\end{array}$

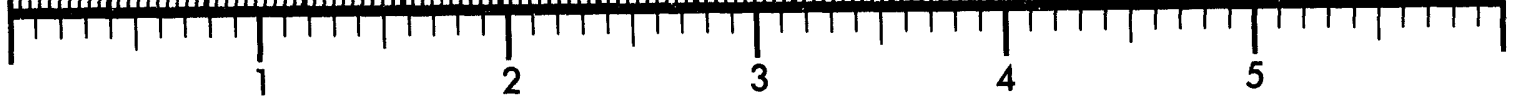

Inches
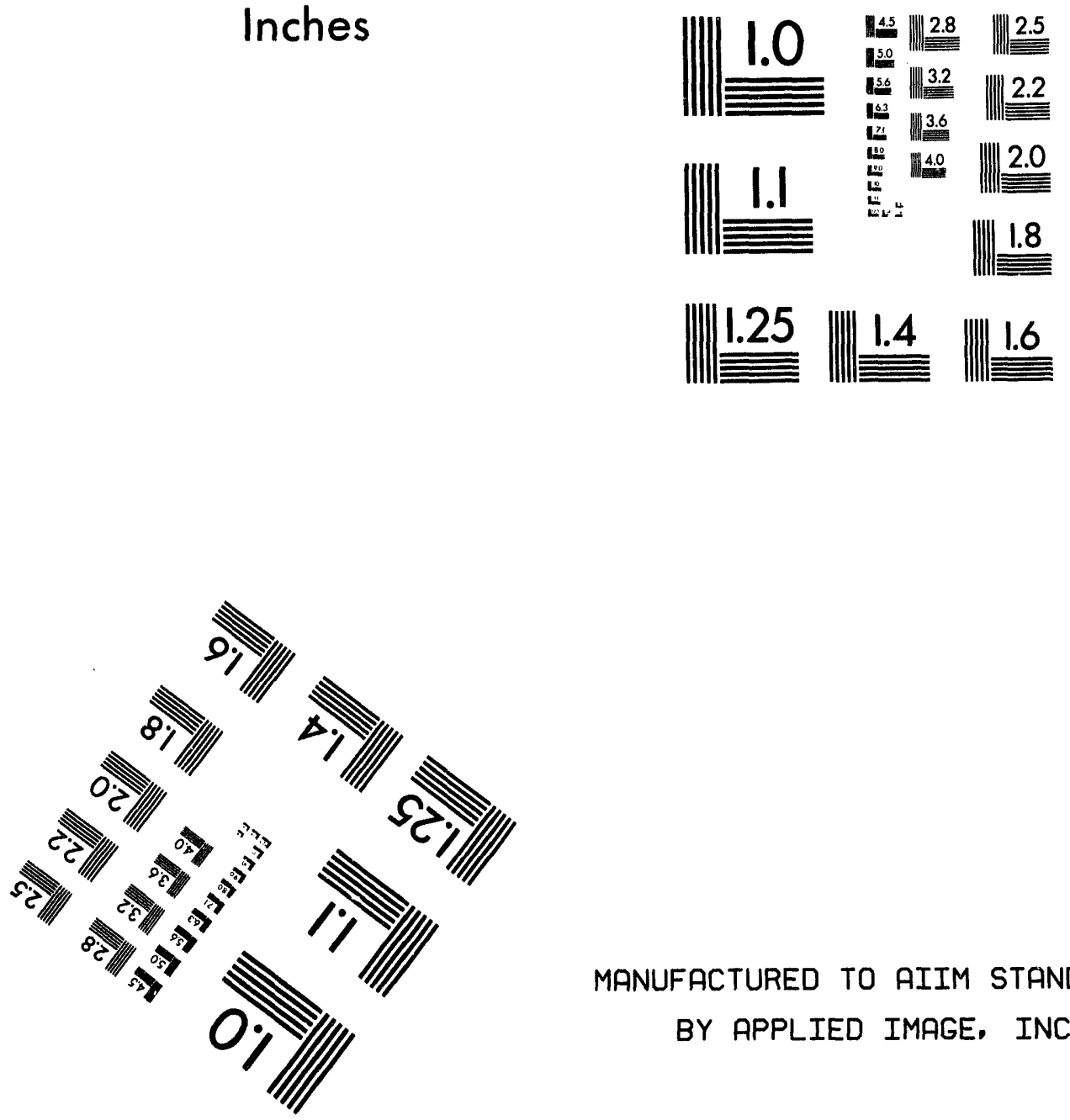

MANUFACTURED TO AIIM STANDARDS

BY APPLIED IMAGE, INC.

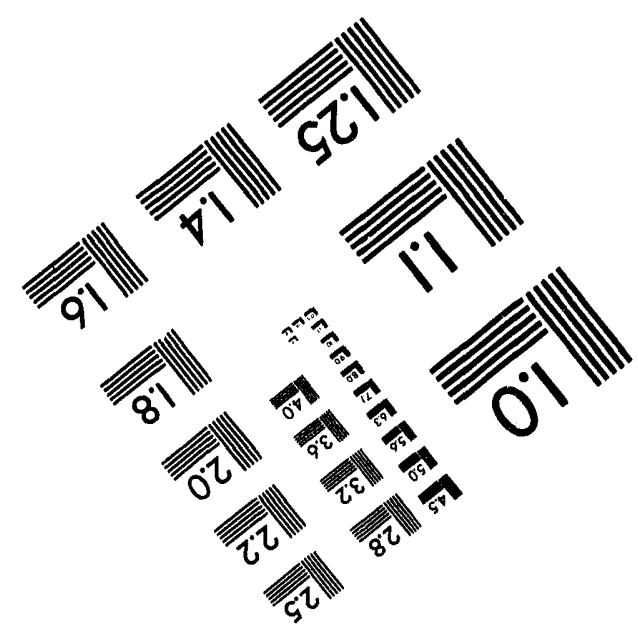



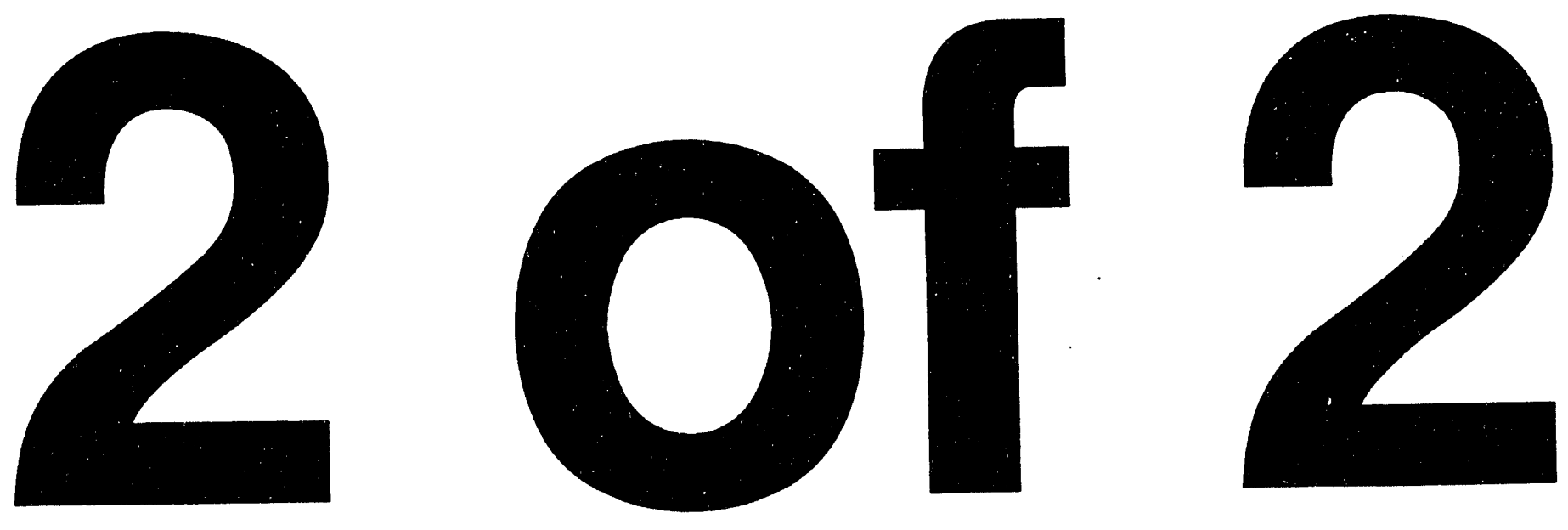
References

Norman, D. A. "The Problem with 'Automation': Inappropriate Feedback and Interaction, Not 'Over-Automation', " Philosophical Transactions of the Royal Society of London, B327, 1990.

NRC, NUREG-1154, Loss of Main and Auxiliary Feedwater at the Davis-Besse Plant on June 9, 1985. U. S. Nuclear Regulatory Commission, Washington, DC 200555, 1985.

NRC, NUREG-1455, Transformer Failure and CommonMode Loss of Instrument Power at Nine Mile Point Unit 2 on August 13, 1991. U. S. Nuclear Regulatory Commission, Washington, DC 20555, October, 1991.

Orasanu, J., R. K. Dismukes and U. Fischer "Decision Errors in the Cockpit," Proceedings of the Human Factors and Ergonomics Society 37th Annual Meeting. Seattle, Washington, October 1993.

Perrow, C., Normal Accidents. Living with High-Risk Technologies, Basic Books, 1984.

Rasmussen, J., Information Processing and Human Machine Interaction: An Approach to Cognitive Engineering. North-Holland, New York, 1986.

Reason, J., Human Error. Cambridge, England: Cambridge University Press, 1990.

Roth, E. M., K. B. Bennett and D. D. Woods, "Human Interaction with an 'Intelligent' Machine". International Journal of Man-Machine Studies, 27, 479-525, 1987.

Roth, E. M., R. J. Mumaw and H. E. Pople, "Enhancing the Training of Cognitive Skills for Improved Human Reliability: Lessons Learned from the Cognitive Environment Simulation Project," in Proceedings of the 1992 IEEE Fifth Conference on Human Factors and Power Plants, 1992.

Roth, E. M., D. D. Woods, and H. E. Pople, "Cognitive Simulation as a Tool for Cognitive Tasks Analysis". Ergonomics, Special Issue on Cognitive Engineering, 35(10), 1163-1198, 1992.
Sarter, N. B. and D.D. Woods, "Situation Awareness: A Critical but Ill-defined Phenomenon". International Journal of Aviation Psychology, 1(1), 43-55, 1991.

Spurgin, A. J., P. Moieni, C. D. Gaddy, G. Parry, D. D. Orivs, J. P. Spurgin, V. Joksimovich, D. P. Gaver, and G. W. Hannaman, Operator Reliability Experiments Using Power Plant Simulators. EPRI NP-6937. Palo Alto, Calif.: Electric Power Research Institute, December, 1989.

Suchman, L. A. Plans and Situated Action: The Problem of Human-Machine Communication. Cambridge: Cambridge University Press, 1987.

Swain, A. D. and H. E. Guttmann, Handbook of Human Reliability Analysis with Emphasis on Nuclear Power Plant Applications. NUREG/CR 1278. Albuquerque, N. M.: Sandia National Laboratories, 1983.

Swezey, R. W. and E. Salas, eds., Teams: Their Training and Performance, New Jersey: Ablex Publishing Corp., 1992.

Trager, Jr., E. A., Case Study Report on Loss of Safety System Function Events. Technical Report AEOD/C504, Office of Analysis and Evaluation of Operational Data, U. S. Nuclear Regulatory Commission, 1985.

Wagenaar, W., and J. Groeneweg, "Accidents at Sea: Multiple Causes and Impossible Consequences," International Journal of ManMachine Studies, 27, 587-598, 1987.

Woods, D. D., L. J. Johannesen, R. I. Cook and N. B. Sarter, Behind Human Error: Cognitive Systems, Computers and Hindsight, CSERIAC State-of-theArt Report, November, 1993.

Woods, D. D., H. E. Pople, and E. M. Roth, The Cognitive Environment Simulation as a Tool for Modeling Human Performance and Reliability, NUREG/CR-5213, U.S. Nuclear Regulatory Commission, Washington, D. C., 1990. 
Woods, D. D. and E. M. Roth, Models of Cognitive Behavior in Nuclear Power Plant Personnel, NUREG/CR-4532, U. S. Nuclear Regulatory Commission, Washington, D. C., 1986.

Woods, D. D., E. M. Roth, and H. E. Pople, Cognitive Environment Simulation: An Artificial Intelligence System for Human Performance Assessment,
NUREG/CR-4862, U. S. Nuclear Regulatory Commission, Washington D. C., 1987.

Woods, D. D., J. A. Wise, and L. F. Hanes. Evaluation of Safety Parameter Display Concepts. Electric Power Research Institute, Palo Alto, CA, 1982. NP-2239. 


\section{Glossary}

Abnormal plant behavior

Adapting procedures

Data-driven moritoring

\section{Diagnosis}

Extra-procedural activity

Knowledge-driven monitoring

Procedure-driven monitoring

Response planning

Response plan monitoring

Situation assessment

Steam space leak
Plant behavior that is not the desired behavior for that mode of operation.

Deviating from the literal statement of procedure steps. It includes taking actions that are not stated in the procedures, not taking actions that are stated in the procedures, and taking actions specified in the procedure but changing them in some way (e.g., changing a plant parameter value mentioned in the procedure.)

Operator monitoring that is triggered by a salient external stimuli such as an alarm.

The process of identifying the cause(s) of abnormal plant behavior.

Operator behavior that is not explicitly directed by a specific procedure step.

Operator monitoring that is driven by an internally generated perceived need for a piece of information.

Operator monitoring that is determined by procedures that include an explicit directive to monitor a particular plant parameter.

The process of deciding on a course of action given a particular situation assessment.

Monitoring the effectiveness of the response plan embodied in the EOPs. Response plan monitoring includes evaluating the consequences of particular actions specified in procedure steps, and evaluating the appropriateness of the EOP procedure path for achieving identified goals.

The process of constructing an explanation to account for observed plant behavior. In the context of NPP operations situation assessment involves developing and updating a mental representation of the factors known or hypothesized to be affecting plant state at a given point in time. Situation assessment refers to both the process of building the mental representation and the resulting mental representation. It is broader than diagnosis in that it encompasses explanations that are generated to account for plant behavior during all plant conditions, including normal as well as abnormal or emergency conditions.

A leak that results in steam escaping from the pressurizer. Examples of steam space leaks are leaking pressurizer PORVs and leaking pressurizer safety valves. 
Glossary

Unexpected plant behavior

Plant behavior that is unaccounted for by the current situation assessment (i.e., by the known or hypothesized factors influencing the plant). Unexpected plant behavior is not necessarily the same as abnormal plant behavior. Plant behavior can be abnormal but not unexpected. For example, in a LOCA, the decrease in pressurizer pressure, would be abnormal but not unexpected. 


\section{APPENDICES}




\section{Appendix A: Detailed Descriptions of Scenarios}

\section{ISLOCA 1}

This scenario, which was run at Plant 1 , is an ISLOCA from the high pressure Reactor Coolant System to the low pressure Residual Heat Removal (RHR) System. Figure 2.3 provides a simple diagram of the systems involved in the scenario. Figure A.1 provides a more detailed diagram of the RHR system. 47

Two isolation valves between the hot leg loop of the RCS system and the RHR system that are normally kept closed and de-energized begin to leak. 48 Specifically these were two isolation valves in series on the suction side of train A of the RHR system. The leak into the RHR produces an increase in pressure in the RHR, which in this scenario resulted in a break in the RHR piping in the Auxiliary Building approximately five minutes into the event (a 2000 gpm leak). This piping break caused reactor coolant fluid to fall to the floor of the Auxiliary Building resulting in Auxiliary Building "misc. sump level high" and radiation alarms.

A key element that makes this ISLOCA event complex is that containment symptoms suggestive of a LOCA inside containment appear early in the event. This complexity occurs because the RHR system includes a relief valve that vents to the PRT which is inside containment. When pressure in the RHR increases, the relief valve opens and fluid is directed to the PRT, which eventually ruptures (approximately three minutes into the event). This action produces radiation alarms within containment suggesting the possibility of a LOCA inside containment. By timing the dynamics of the event carefully it was possible to create a situation where the EOP directed the

${ }^{47}$ Figure A.1 is a partial schematic of the RHR system. For simplification some components have been omitted.

${ }^{48}$ In the postulated event the valve seats on the suction valves fail, causing leakage into the RHR system. To increase the credibility of the event we posiulate that the valve seat on one of the suction valves had failed earlier without being detected and that the valve seat on the second suction valve failed at the initiation of the event. operators to the LOCA inside containment procedure before they got to the point in the EOP procedure that checks for ISLOCA symptoms. Once the operators are in the LOCA procedure there is no explicit procedure transition that allows them to get to the ISLOCA procedure. ${ }^{49}$ A diagram of the relevant EOP procedures and transitions for the plant at which the scenario was run is provided in Figure A.2.

The first alarms that come in are an RHR discharge pressure high alarm and pressurizer pressure and level low alarms. This results in a reactor trip that occurs approximately 30 seconds later. At that point the crew is required to turn to the Reactor Trip and SI Procedure (E-0) in the EOPs (see Figure A.2). They reach a step in the procedure that asks if the RCS is intact. By that point the PRT has ruptured, resulting in radiation alarms inside containment. Therefore, the answer is no, and the EOPs direct a transition to the Loss of Reactor or Secondary Coolant Procedure (E-1). There is a step later in the E-0 procedure that checks for Auxiliary Building radiation symptoms and if the answer is yes directs them to an ISLOCA procedure, but the operators transition to $\mathrm{E}-1$ before they get to that step. Once in E-1 there is no explicit transition to the ISLOCA procedure. There is a step that checks for Auxiliary Building radiation. By the time the operators reach that step they do have Auxiliary
${ }^{49}$ It should be noted that, when we ran this event, there was no explicit transition from the LOCA procedure to the ISLOCA procedure in the EOPs used at that plant, the latest version of the Emergency Response Guidelines (ERGs) includes an explicit transition from the LOCA procedure to the ISLOCA procedure. 


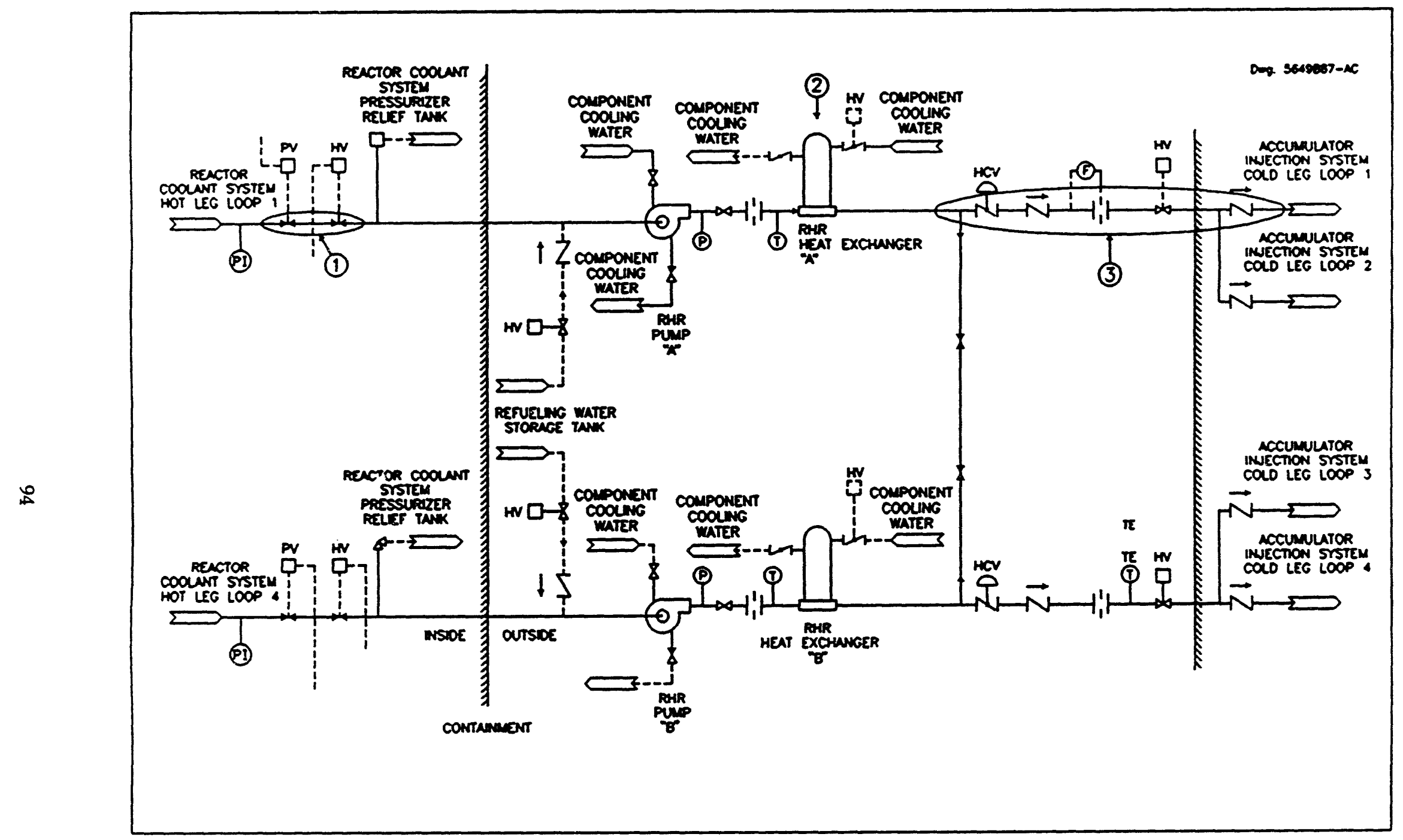

Figure A.1 More detailed diagram of the Residual Heat Removal System. (1) The two isolation valves between the Reactor Coolant System Hot Leg Loop and the RHR that leaked. (2) The RHR heat exchanger with the Component Cooling Water system that broke in ISLOCA 2. (3) The series of check valves between the Reactor Coolant System Cold Leg Loop and the RHR system. Back leakage through those check valves provided an alternative hypothesis to explain the inflow of RCS fluid into the RHR system. 


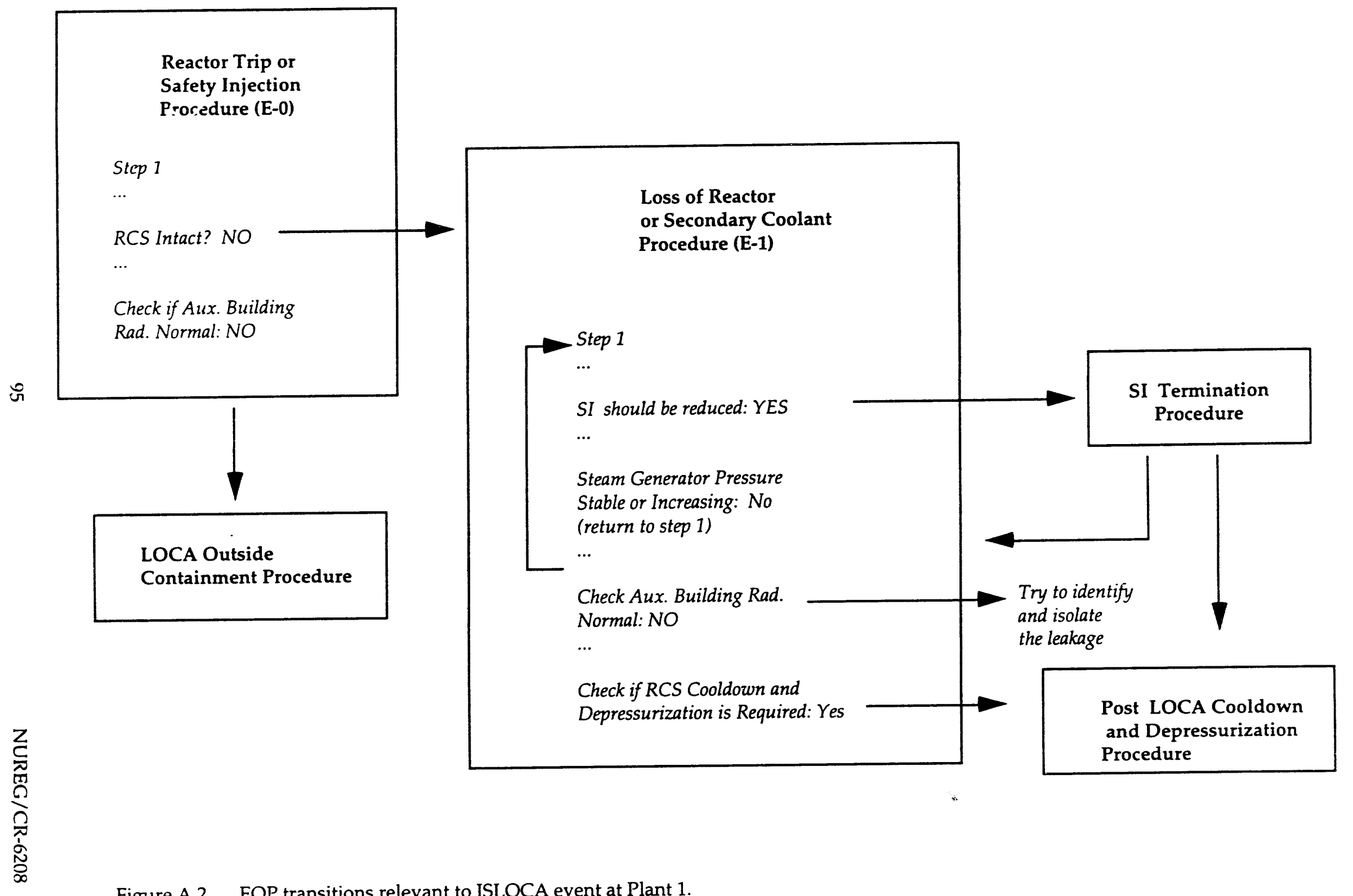

Figure A.2 EOP transitions relevant to ISLOCA event at Plant 1. 
Building symptoms. In that case the procedure directs them to "identify and isolate the leakage." Thus at that point they are explicitly required to diagnose the source of the leak and take action to terminate it on their own. The next steps in the procedure specify a transition to a Post-LOCA Cooldown and Depressurization Procedure in order to depressurize and cooldown the RCS and reduce the leak rate.

By the time radiation symptoms appear in the Auxiliary Building, the primary symptoms of an RHR problem (i.e., RHR discharge pressure and temperature high) are gone because the break into the Auxiliary Building relieves the pressure buildup in the RHR. Therefore, if the crews did not detect the RHR discharge pressure alarm early in the event or observe the RHR discharge pressure or temperature symptoms, they had few clues as to the source of the RCS leak into the Auxiliary Building.

The only other piece of evidence that the source of the leak is the RHR system is the pressure buildup in the PRT and its subsequent break. If the crew pursues potential sources of input into the PRT, it would lead them to suspect the RHR system, since other potential sources of input to the PRT read closed and had no reason to open (e.g., pressurizer PORV and safeties), and/or are too small to generate the pressure buildup in the PRT that was observed (e.g., CVCS letdown relief; seal water return).

The combination of evidence of buildup of pressure in the PRT and radiation in the Auxiliary Building can be simply explained by an RHR problem. The alternative is to postulate two independent problems to explain the PRT symptoms and the Auxiliary Building symptoms, which is less plausible.

Later in the event the crews are given a stronger clue that the problem is in the RHR system.

Approximately 15-20 minutes into the event, they receive a call from the Auxiliary Building indicating that the sumps outside the RHR pump room are flooded.

Once the operators identify a leak into the RHR the 'e are two plausible hypotheses for the source of the leak. One is a failure of the two isolation valves between the hot leg loop of the RCS system and the RHR on the suction side of the RHR pump. This is the event that we postulated. Given this hypothesis the actions required to isolate the leak are to call to the
Auxiliary Building to request that the valves be reenergized, to verify that they are closed, and to close them if they are not. In the scenario as we constructed it, the valves read closed but were leaking. If the operators called to check on the status of these isolation valves they were told that the valves read closed. In this scenario, the leak is unisolatable and the best course of action is to proceed with the cooldown and depressurization, while conserving Refueling Water Storage Tank (RWST) level.

An alternative plausible hypothesis that would account for all of the evidence available is that there is leak back from the RCS through a series of failed check valves (See Figure A.1). Given this hypothesis, the leak could be isolated by closing an isolation valve on the discharge side of the RHR pump that is normally kept open. This valve isolates the RHR system from the Accumulator Injection System Cold Leg Loops. Since the failed check valve hypothesis was determined by the instructors at Plant 1 to be equally plausible, in some cases, when crews closed the isolation valve on the discharge side of the RHR pump, the instructors terminated the leak.

\section{ISLOCA 2}

ISLOCA 2 differed from ISLOCA 1 in three main respects. First, the RHR discharge pressure high alarm, which provided the primary indicator of a problem in the RHR in ISLOCA 1, was suppressed in ISLOCA 2. This change removed the primary indicator of a leak into the RHR system. Second, the leak into the RHR system led to a break in the RHR heat exchanger to the $\mathrm{CCW}$ system. This break caused abnormal radiation symptoms in the CCW system. These two factors combined meant that more active search and integration of evidence was required to identify the problem in the RHR and connect the problem in the RHR system with the problem in the CCW system.

The third difference between ISLOCA 1 and ISLOCA 2 was in the procedures available. In the case of ISLOCA 2, the LOCA procedure included a step to transition to the ISLOCA procedure if there was high radiation in the Auxiliary Building (see Figure A.3). However, because of the dynamics of the event, in some cases the transition step was skipped. In other cases the criteria for transitioning to the ISLOCA procedure were not met when that step was reached. 


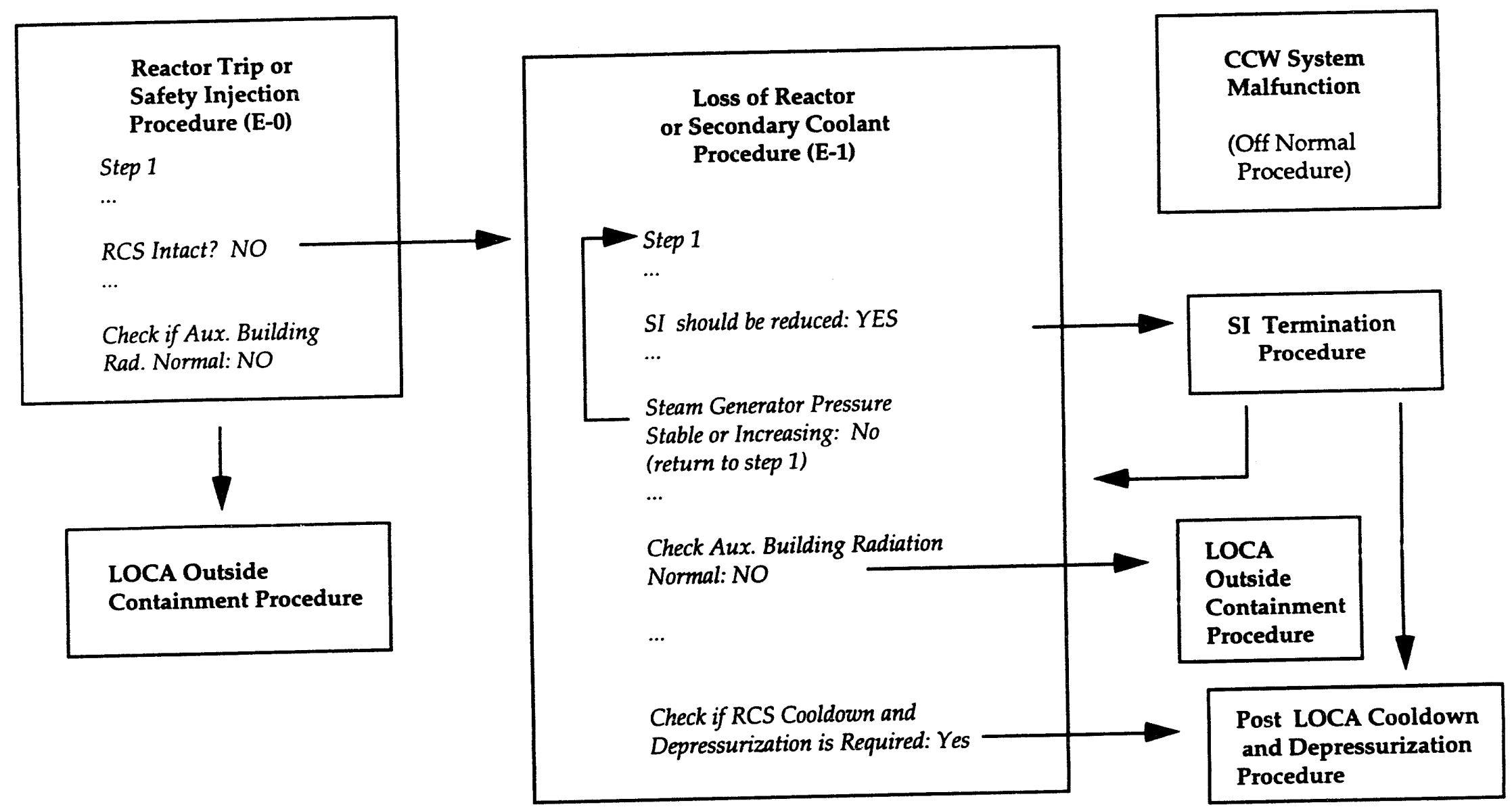

Figure A.3 EOP transitions relevant to ISLOCA event at Plant 2. 
This plan allowed us to examine crew performance in cases where the procedure containing relevant guidance could not be reached within the EOP transition network.

As in the case of ISLOCA 1, two isolation valves between the hot leg loop of the RCS system and the RHR system that are normally kept closed and deenergized were failed open. ${ }^{50}$ Specifically, these were two isolation valves in series on the suction side of train B of the RHR system. Pressure in the "B" train RHR system increases to the point where the suction relief valve begins to relieve to the PRT. Eventually, the PRT ruptures (within approximately five minutes of the reactor trip) causing high radiation levels inside containment.

The pressure buildup in the RHR eventually causes a tube in the RHR heat exchanger to the CCW to break, causing a high $\mathrm{CCW}$ radiation alarm to come in, followed by indications that the CCW Surge Tank level is rapidly increasing. Rupture of the RHR heat exchanger tube reduces RHR pressure to below the lift setpoint of the RHR suction relief valve, thereby causing the LOCA to be redirected to the CCW System. Eventually the CCW Surge Tanks overflow causing reactor coolant fluid to spill onto the floor of the Auxiliary Building, resulting in Auxiliary

Building alarms. 51

Several factors made ISLOCA 2 diagnostically more challenging than ISLOCA 1 . First, the RHR high discharge pressure alarms were suppressed, so that crews did not get an early indication of an RHR problem. 52 Second, greater understanding of the system interconnections and potential flowpaths among the systems is required to connect the symptoms observed in the different systems. A complete situation assessment required that the crews identify:

${ }^{50}$ As in the case of Plant 1, although the two valves read closed they were stuck open and leaked.

${ }^{51}$ The simulated scenario was timed so that Auxiliary Building radiation symptoms did not appear until after the crews passed the step in E-1 that checks for Auxiliary Building symptoms. This was the case for all but one of the crews.

52 The fact that the RHR discharge pressure high alarm did not come on, is an important difference between how the event was run at Plant 1 and at Plant 2.
- the flow path by which contaminated reactor coolant fluid reached the CCW system (i.e., contaminated water from the reactor coolant system reaching the CCW system via the RHR system);

- the source of pressure buildup in the PRT (i.e., via the RHR relief valve);

- the source of radiation in containment (i.e., the break in the PRT);

- the source of radiation in the Auxiliary Building (i.e., the overflowing CCW surge tanks).

It also required that they recognize that all these symptoms are due to a single underlying fault (i.e., an RCS leak into the RHR).

The first alarms that came in were the pressurizer level and pressure low alarms, and the PRT level and pressure high alarms. These alarms were followed within approximately 20 seconds by a Reactor Trip.

The primary clue pointing to an RHR problem was the increase in PRT pressure and subsequent break of the PRT. If crews searched for possible sources of input into the PRT, it could lead them to identify the abnormal RHR discharge pressure. There is an RHR discharge pressure meter in the control room. If the crew had looked at the meter before the break in the RHR heat exchanger occurred, they would have seen that it read abnormally high. However, there were no alarms or procedure steps that would direct the operator to look at that meter.

Another difference between ISLOCA 1 and ISLOCA 2 is in the procedures available to support the operators. While overall the EOPs at Plant 1 and Plant 2 were similar in structure and content, there were some differences. Figure A. 3 provides a graphic representation of the relevant procedures at Plant 2. There are two main differences to note. One difference is in the step in the Loss of Reactor or Secondary Coolant Procedure (E-1) that checks for Auxiliary Building radiation symptoms. At Plant 2, if the Auxiliary Building radiation is detected, the operators are directed to the LOCA outside containment procedure, whereas at Plant 1 , the operators were directed to identify and isolate the leak on their own. In principle, this means that the crews at Plant 2 had a procedure path by which to get 
to the ISLOCA procedure from the Loss of Reactor or Secondary Coolant Procedure (E-1). However, due to the dynamics of the event, that transition was not always accessible. One reason is that it was possible to transition out of the E-1 procedure to the SI

Termination procedure before reaching the step in E-1 that checks for Auxiliary Building radiation. As is shown in Figure A.3, it was possible to transition from E-1 to the SI Termination procedure before the Auxiliary Building radiation step was reached, and then to transicion directly from the SI Termination procedure to the Post-LOCA Cooldown and Depressurization Procedure. In this way the step checking for Auxiliary Building radiation was never reached.

It was also possible that when the step checking for Auxiliary Building radiation in E-1 was reached there was no radiation in the Auxiliary Building because the CCW surge tank had not yet overflowed. As a result the criteria for transitioning to the ISLOCA procedure were not met.

Thus, as in the ISLOCA 1 case, the dynamics of the event created a situation for many of the crews where there was no procedurally directed way to reach the ISLOCA procedure.

A second difference in terms of procedures available, is that at Plant 2 there was an abnormal operating procedure available to support identifying and isolating the leak into the CCW: CCW System Malfunction procedure. While this procedure is not part of the EOPs, it could be consulted. This procedure provides a clearly laid out logic for identify:ng and isolating the source of an outside leak into the CCW. If the crews chose to consult that procedure, and followed it correctly, it would enable them to localize the problem to the RHR heat exchanger. We were interested in whether crew members would consult the off normal procedure for guidance, and if not, whether they would use a similar line of reasoning in identifying and isolating the leak.

Figure A.4 provides a graphic representation of the main logic of the CCW System Malfunction procedure. The procedure begins by checking whether the level in the CCW surge tanks is going up. If, as in this case, the CCW surge tank level is going up, it indicates a leak into the CCW. The procedure then checks whether the service loop (i.e., the systems being serviced by the $\mathrm{CCW}$ ) is aligned to the affected $\mathrm{CCW}$ train. If it is, as was the case in this scenario, the procedure checks for possible sources of leakage into the $\mathrm{CCW}$ from the service loop (e.g., excess letdown heat exchanger, RCP Thermal Barriers). It then has the operators transfer the service loop to the unaffected $\mathrm{CCW}$ train. The procedure then checks whether level continues to go up in the CCW train that was previously aligned to the service loop. If the CCW surge tank level continues to go up, it means that the in leakage is not from the service loop. It can then be concluded that the in-leakage is from the Safety Train - specifically the RHR heat exchanger. The procedure then directs the operators to isolate the RHR heat exchanger but does not indicate which valves to close. In fact, there are two valves that can be closed to isolate the RHR heat exchanger. One valve is on the $\mathrm{CCW}$ side of the hert exchanger. A second valve is on the RHR side of the heat exchanger (between the discharge side of the RHR pump and the heat exchanger).

\section{Loss of Heat Sink 1 (LHS 1)}

The Loss of Heat Sink event involved a total loss of feedwater flow complicated by a leaking pressurizer PORV. Figure 2.4 provides a simple diagram of the systems involved in the scenario.

In the simulated event both the auxiliary and main feedwater systems are made unavailable so that operators are forced to use the condensate system to supply feedwater. This change requires that they depressurize the RCS and block SI signals. The auxiliary spray valve is shut so that the operators have to use the pressurizer PORV to depressurize the RCS. After they open and close the pressurizer PORV, it starts to leak (though the pressurizer PORV indicator reads closed). One of the key features of this situation is that SI is blocked and must be started manually to deal with the PORV leak. However, the main focus of operator attention and the procedures is on recovering a secondary heat sink. This creates a situation that allows us to examine how operators discover and handle an unexpected second fault. 


\section{CCW System Malfunction}

Step 1

$\cdots$

Determine if the in-leakage is from the service loop and attempt to isolate:

- excess letdown heat exchanger

- letdown heat exchanger

- $R C P$ thermal barriers

Transfer the Service Loop to the unaffected train

Check CCW surge tank level in both trains

If level continues to increase uncontrollably on safety train previously on service then isolate leak on Safety train:

- RHR heat exchanger

$\cdots$

Figure A.4 Structure of Off Normal Procedure (OFN) for CCW System Malfunction.

In this version of the event the crews are never given feedwater back. As a result they remain in the Loss of Secondary Heat Sink procedure where the only guidance available for dealing with the leaking PORV is in a caution that states that manual initiation of SI may be required if plant conditions degrade.

At the start of the scenario the plant is at $50 \%$ power. The crews are told that the $B$ motor-driven auxiliary feedpump and the motor-driven main feed startup pump are tagged as being out of service. The crews are asked to increase load at $10 \%$ per hour. Five. minutes into the event, an earthquake occurs resulting in a reactor trip. This automatically causes the main feedwater pumps to trip and the auxiliary feedwater system to come on. At this point the crew is required to go to the Reactor Trip and SI procedure in the EOP $(E-0)$. Four minutes later a turbine-driven auxiliary feedwater pump high temperature alarm comes on. Three minutes after that the turbine-driven auxiliary feedwater pump trips. At this point the crew is in a total loss of feedwater event.

According to the rules of usage of the EOPs, the crews are required to go through the E-0 until they are transitioned to an emergency guideline procedure. 
Because the reactor trip was due to an earthquake, the transition from E-0 is to a Reactor Trip response procedure. Once they are transitioned to an emergency guideline procedure, the STA is required to begin monitoring five prioritized critical safety functions: subcriticality; core cooling; heat sink; integrity; and containment. Since all feedflow is lost, the heat sink safety function is violated, and the crews are directed to the Loss of Secondary Heat Sink function restoration guideline. Figure A.5 provides a diagram of the relevant procedures and procedure transitions for this event.

Function Restoration Guidelines (FRG) are intended to restore critical safety functions and thus they have a special status. Crews are required to stay within the FRG procedure until the critical safety function is recovered or violation of a higher priority safety function is identified.

The Loss of Secondary Heat Sink procedure guides the crew to attempt to restore feedwater through a number of alternative means. These attempts require extensive interaction via phone communication with personnel outside the control room. In the training simulator, these interactions are simulated by having training instructors act as auxiliary crew.

The procedure directs the operators to first attempt to re-establish auxiliary feedwater flow. If this attempt fails the operators are directed to try to establish main feedwater flow. If this fails they are directed to try to establish feedwater flow through the condensate system. As a means of last resort the crews are directed to use bleed and feed to provide cooling. This method involves initiating SI and then opening the pressurizer PORV. Since this method involves intentionally creating a break in the RCS system it is the least preferred alternative. The procedure specifies that if at any point in the event wide range level in any three steam generators (SGs) is less than a specified value, or pressurizer pressure is greater than or equal to a criterion value due to the loss of heat sink, then a bleed and feed must be initiated immediately. 53

In this event feedwater is never re-established. Since we did not want the crews to go to a bleed and feed

53 The values vary from plant to plant. procedure, initiating plant parameters were tuned so that SG levels never reached bleed and feed criteria.

The procedure first has the operators try to reestablish auxiliary feedwater flow. These attempts fail. (The crew is told that the turbine-driven auxiliary feedpump has a bearing problem and that the motordriven auxiliary feedpump has a breaker problem and that they will take an extended period to fix.) The procedure then directs the crew to attempt to start the main feedwater pumps. This attempt fails as well. The pumps trip, and the crew is told that they cannot be restarted.

At this point the procedure directs the operators to try to establish feedwater via the Condensate System. A number of steps are required to establish feedflow through this system. Because the condensate pumps operate at a lower pressure, the SGs need to be depressurized. ${ }^{54}$ Before this can be done, the RCS system needs to be depressurized to less than 1920 psig. This is accomplished using either the auxiliary spray or the pressurizer PORV. In the event as we ran it, the auxiliary spray failed to come on so that the crew was forced to use the pressurizer PORV. In this event when the PORV is closed, it never completely reseats. As a result, although it reads closed, there is a small leak from the pressurizer PORV from this point on in the event. 55

As part of this step in the procedure the crews are also required to block signals for automatic actuation of SI. This is done to avoid spurious SI when the steam generators are depressurized. 56 This action has potentially serious consequences since it means that a major automatic safety actuation system is no longer in operation and must be manually initiated if needed. To emphasize this point, a caution appears prior to the step directing the crew to block SI that states, " Following block of automatic SI actuation,

\footnotetext{
54 The exact SG pressure value varies from plant to plant but is in the range of $550 \mathrm{psig}$.
}

55 A 5\% PORV leak with a 500 second ramp was used at Plant 1. The leak was inserted at the point when the crew started to depressurize a steam generator.

56 SI signals for Low steamline pressure and low pressurizer pressure are blocked. 
manual SI actuation may be required if conditions degrade."

The event was specifically designed to place the crew in a situation where a leak through the pressurizer PORV would cause RCS conditions to degrade. A goal was to determine whether crews would choose to manually safety inject.

Once the RCS is depressurized, and the SI signals are blocked, the crews are directed to depressurize at least one SG and then establish condensate flow to that SG. Establishing a condensate flow path involves a number of steps that require actions to be taken by auxiliary operators. First, jumpers must be used to bypass the fast close action on the feedwater isolation valves. This bypass requires that Instrumentation and Control technicians come in and physically jumper the valves in the control room. An equipment operator must then be dispatched to manually jack open the feedwater regulating valve for the desired steam generator. In addition, a discharge valve on at least one of the main feed pumps must be opened.

In this event we did not want feedwater to be reestablished. This situation was accomplished by introducing delays in getting the feedwater regulating valves open. Examples include auxiliary operators going to the wrong valve (the feedwater isolation valve instead of the feedwater regulating valve); auxiliary operators being detained by health physics because of having been exposed to radiation; auxiliary operators being unable to manually jack open the valves; and auxiliary operators breaking the feedwater regulator valve actuators. As a result of these delay tactics a great deal of operator activity involved calling the operators in the Auxiliary Building for status reports and receiving calls.

The Loss of Heat Sink procedure focuses operator attention on the secondary side and recovery of feedwater. In the meantime there is a secondary fault: a leak on the primary side through the pressurizer PORV that manifests itself in a number of ways.

The first symptoms are a decrease in pressurizer level and pressure. Since the crews are depressurizing the SG around this time, at first the pressurizer level and pressure behavior can be attributed to a cooldown caused by depressurizing the SG. Once the SG depressurization is completed, however, the pressurizer behavior can no longer be attributed to cooldown due to secondary side activity.

At some point both the pressurizer level and the pressure decrease sufficiently that subcooling is lost. At that point, RCS begins to become superheated and a bubble forms in the reactor vessel. As a result the level in the pressurizer starts to go up, while the level in the reactor vessel starts to go down. The reactor vessel level indicator (RVLIS) in the control room provides an indication of reactor vessel level. $A$ RVLIS value of $100 \%$ or less with pressurizer level going up indicates a bubble in the reactor vessel. The pressurizer level going up while the pressurizer pressure continues to decrease provides a similar indication. This combination of symptoms cannot be explained by a cooldown. They can only be explained by a leak out of the pressurizer: Possibilities include a leak out of the pressurizer PORVs or a leak out of the pressurizer safety valves. These are collectively referred to as a steam space leak. Since the pressurizer PORVs read closed, there is no direct evidence to discriminate between a leaking PORV hypothesis and a leaking safety valve hypothesis.

If the leak is not terminated, pressurizer level continues to go up and the pressurizer eventually becomes completely filled with water (i.e., goes solid).

Another indication of a steam space leak is activity in the PRT. Since the pressurizer PORV is opened to depressurize the RCS, some activity in the PRT is expected. However, since the PORV continues to leak into the PRT, symptoms continue to increase even after the PORV is closed. Eventually, the PRT ruptures resulting in radiation symptoms in containment. The rupture of the PRT cannot be explained by the cooldown hypothesis.

One set of questions in this scenario concerned identification by the crews of a problem in the RCS. A second set of questions concerned what actions, if any, crews decide to take to deal with the leak in the RCS. One option available is to close the PORV block valve. This would terminate the leak. 57 Another

\footnotetext{
${ }^{57}$ In this scenario, closing the block valve did not terminate the leak for all crews. In some cases the leak was continued (postulating a leak in the block valve as well) in order to allow us to observe whether the crews would choose to manually safety inject as RCS conditions continued to degrade.
} 
option is to initiate a manual SI. While the Loss of Heat Sink procedure did not include an explicit criterion for a manual SI, it did include the caution that manual SI actuation may be required if conditions degrade. This caution was intended to allow crews to manually initiate SI at their discretion.

Given an undetected or uncontrollable steam space leak, there are several arguments in favor of manual initiation of SI. First, the leak through the pressurizer PORV is a LOCA. Unless the leak is isolated (e.g., by closing the pressurizer PORV block valve) an SI will eventually be required. If the crew successfully recovered feedwater they would be transitioned back to the procedure they were following. Once in that procedure, they would immediately meet the criteria for manual initiation of SI. If the crew did not recover feedwater, they would eventually meet the criteria for a bleed and feed, which itself involves initiating SI; thus whether feedwater is recovered or not, unless the leak is terminated, the crew would eventually have to initiate SI.

There are also arguments against manual initiation of SI. One is that there is no specific procedure directive to initiate SI. Another argument is that initiating SI could result in delay of recovery of feedwater, possibly causing the crew to have to go to a bleed and feed which is undesirable.

If the operators take no action on their own, conditions in the RCS will continue to degrade.

Eventually, reactor vessel level would decrease to less than $40 \%$. At that point, based on the "red path" core cooling critical safety function criteria, the EOPs would direct the operators to a core cooling function restoration procedure designed to respond to loss of core cooling. However, by that point conditions in the RCS would be significantly degraded with increased risk of core damage.

Once the crew identified a problem on the primary side, and determined a course of action, if any, the simulation was terminated. As a result the simulation was terminated before the loss of core cooling safety function "red path" criterion was reached.

\section{Loss of Heat Sink 2 (LHS 2)}

Loss of Heat Sink 2 was similar in most respects to the Loss of Heat Sink 1 scenario. There was a total loss of feedwater flow complicated by a stuck open pressurizer PORV. As in LHS 1, the crews were unable to recover either auxiliary feedflow or main feedflow. As a result they had to use the condensate system. However, LHS 2 differed in two essential respects from LHS 1 . First, if the crews decided to close the PORV block valve the leak was terminated. Second, eventually the crews were allowed to recover feedwater and return to the procedure which they had been following, which in this case, was the Reactor Trip Response procedure.

The fact that the crews transitioned to the Reactor Trip Response procedure provided the opportunity to observe $l$ ow crews responded when they reached a step in the procedure that appeared inappropriate for the current situation. Several steps in the Reactor Trip Response procedure were inappropriate to follow literally given that they had just transitioned from a Loss of Heat Sink procedure and that they were using the condensate system to feed the steam generators. These steps required the operators to reverse actions that they had intentionally taken as part of the LHS procedure. EOP background documents explicitly recognize that some of the steps may be inappropriate when returning from a critical function restoration procedure, such as the Loss of Heat Sink procedure, and state that operator judgment may be needed under these circumstances. We wished to evaluate how the crews responded to these steps.

The initiating conditions for LHS 2 also varied slightly from LHS 1. As in LHS 1, at the start of the scenario the plant was at $50 \%$ power. The crews were told that the "B" essential service water, the " $B$ " auxiliary feedwater pump, and the " $\mathrm{B}$ " diesel generator were out of service.

The main steam isolation valves inadvertently close causing a reactor trip. This action plays the role that the earthquake played in LHS 1 . The crews go to the Reactor Trip and SI procedure in the EOP (E-0). When the turbine driven auxiliary feedwater pump starts, the coupling between the turbine and the pump fails, preventing feedflow. The " $A$ " motor-driven auxiliary feedwater pump also fails due to a seized bearing. This action results in a total loss of feedwater.

The crews continue through E-0 until they are transitioned to the Reactor Trip Response procedure. At that point the STA begins monitoring critical safety 
functions. Since all feedflow is lost, the heat sink safety function is violated, and the crews are directed to the Loss of Secondary Heat Sink function restoration guideline. Figure $A \mp$ provides a diagram of the relevant procedures and procedure transitions for this event.

The procedure first has the operators try to reestablish auxiliary feedwater flow. These attempts fail. The procedure then directs the crew to attempt to start the main feedwater pumps. This attempt fails as well. The main feedwater pumps are tripped and cannot be reset.

At this point the procedure directs the operators to try to establish feedwater via the Condensate System. This step requires the RCS system to be depressurized to less than 1920 psig. As in LHS 1, auxiliary spray fails to come on so the crew will have to use the pressurizer PORV. When the PORV is closed, it never completely reseats. As a result, although it reads closed there is a small leak from the pressurizer PORV from this point on in the event. However, if the crew closes the pressurizer PORV block valve the leak is terminated.

As in the LHS 1 event the EOPs direct the crews to block SI signals. A caution appears immediately prior to this step indicating that manual initiation of SI may be required as conditions degrade.

Once the RCS is depressurized, and the SI signals are blocked, the crews are directed to depressurize at least one SG and then establish condensate flow to that SG. This sequence involved the same activities as in LHS 1. The one difference is that the feedwater regulating valves are eventually opened and feedwater is restored.
Once feedwater is restored the Loss of Heat Sink procedure directs the crews to return to the procedure that was in effect when feedwater was lost. In this case it was the Reactor Trip Response procedure. The Reactor Trip Response procedure includes a number of steps that are not applicable given that the steam generators are being fed via the condensate system. Figure A.5 specifies these steps.

One step asks the crew to verify that the feedwater isolation valves are closed. If they are not the EOP specifies that they should be closed. In fact, the flow of feedwater through the condensate system requires that the valves be open.

Another step has the crew check that pressurizer pressure is greater than 1830 psig. If it is not, the step directs the crew to manually actuate SI. The pressurizer had been intentionally depressurized to less than 1920 as part of the Loss of Heat Sink procedure. Pressurizer pressure tended to be below 1830 psig, partly due to cooldown, when that step in the Reactor Trip Response procedure was reached. However, a manual initiation of SI would have been inappropriate in these circumstances.

Finally, the foldout page for the Reactor Trip Response procedure specified criteria at which SI should be immediately actuated. These criteria were: (1) if pressurizer level cannot be maintained greater than $4 \%$ or (2) if RCS subcooling was less than 30 degrees $F$. When the crews returned to the Reactor Trip Response procedure from the Loss of Heat Sink procedure, in many cases pressurizer level was less than $4 \%$ due to cooldown. The feedflow into the steam generator from the condensate system resulted in a large cooldown on the primary side which caused a shrink in pressurizer level. While the pressurizer level met the SI criteria, the behavior of the pressurizer was not abnormal under those conditions. 


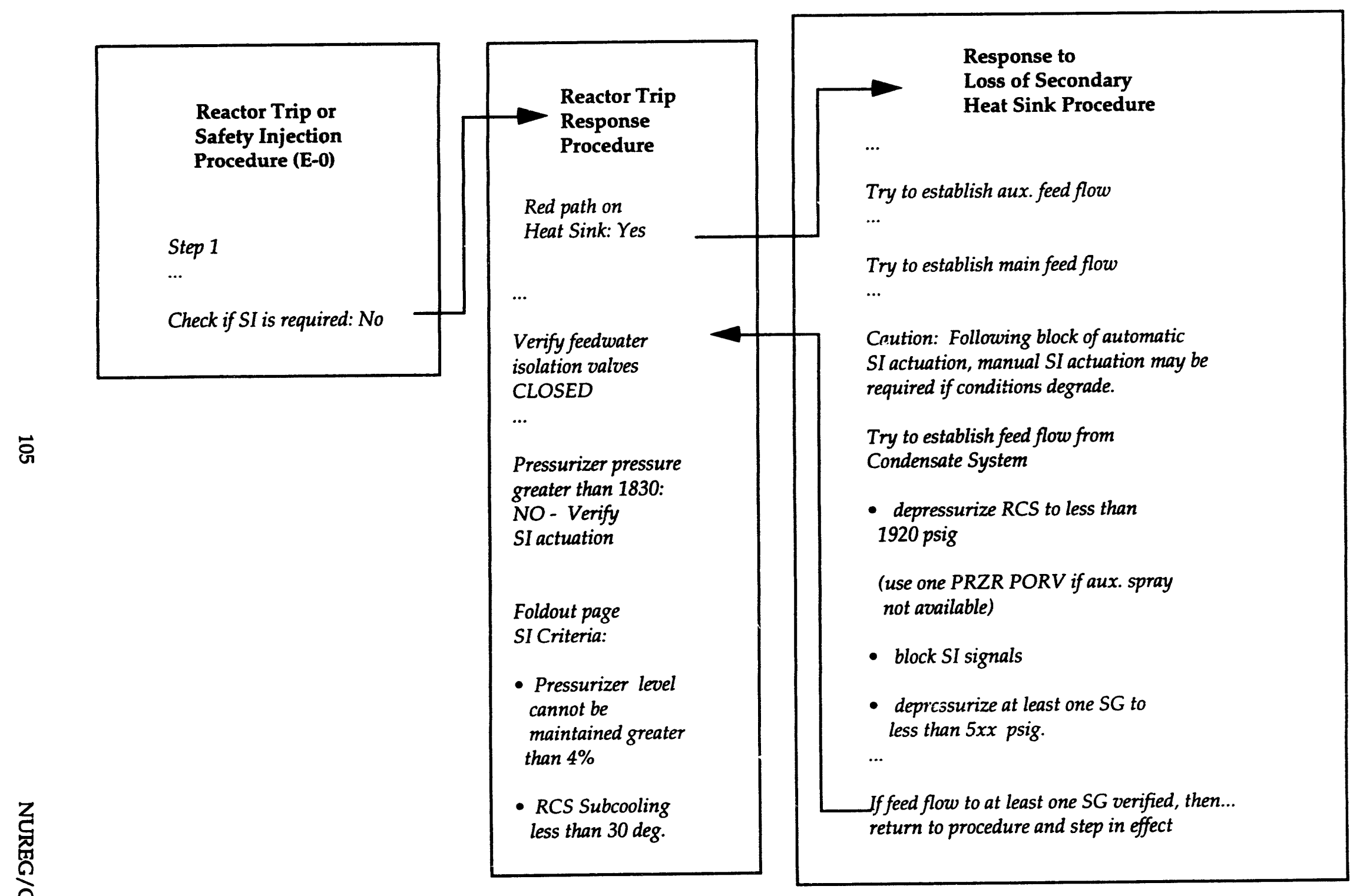

Figure A.5 EOP transitions for Loss of Heat Sink event. The Reactor Trip procedure is from Plant 2 and was used in the LHS 2 scenario. 
Appendix B: Instructions to Study Participants and Sample Summary Sheets 
Instructions

\section{Instructions to Study Participants}

The simulator exercises you are about to participate in will be used as part of a research project being conducted by the Westinghouse Science \& Technology Center for the NRC's Research Office.

The purpose of the research is to understand the decision making process involved in diagnosing and responding to challenging postulated accident scenarios. Many of the actual nuclear power plant incidents, for example, Three Mile Island, have had twists or complications that made the events challenging to handle. As a result, the research team is interested in how operators use their knowledge, training, procedures and any other resources available to them in handling similar situations. They are trying to develop a computer simulation that responds to accident scenarios much as operating crews would. The long term goal is to use this computer simulation to predict situations that are likely to be challenging, and to help define aids - training, procedures, or control room displays -- that could help operators in these situations.

The members of the research team are:

Dr. Emilie M. Roth, a human factors psychologist at the Westinghouse Science \& Technology Center and principal investigator on this project.

Dr. Harry Pople, Jr., a computer scientist from the University of Pittsburgh and the developer of the computer simulation.

Dr. Roth and Dr. Pople are contractors to the NRC for this research project. Dr. Paul M. Lewis, of the NRC Office of Nuclear Regulatory Research, is the contract monitor for the project. His role is to ensure that the activities of the research contractors remain within the scope of the contract.

Today you will be participating in two simulated accident scenarios that have been designed to be challenging. Handle these events as you would if they were actually happening in the plant. Use all of the resources available to you - anything you would use in a real situation to mitigate the event.

It is important to emphasize that this is strictly a research project and you are not being evaluated. The events are expected to be challenging and performance may not always be successful. Any problems that may occur are expected. There are no pass/fail criteria, merely an observation of your decision making process. The results of these exercises are not to be used as a means for evaluating individual operators, operating crews, and the plant. This is understood and agreed to by Westinghouse, the NRC, and the utility management.

As part of this research program, data will be collected on how actual operating crews respond to challenging scenarios. The idea is to understand how operators handle these events and to use that as input in developing and testing the simulation program that is being built.

The simulator scenarios will be videotaped so that they can be reviewed later to understand what happened in more detail. The videotapes will belong to the plant and are being made solely for this research project. They will be borrowed by Westinghouse for analysis and then returned directly to the plant. According to the agreement with the plant, the only people who will see the tapes are the Westinghouse researchers and Dr. Paul Lewis. No one else will be provided access to the tapes without explicit permission from the plant.

The research team will largely rely on the communication among the crew to keep track of the event. They are especially interested in your thoughts about what is happening to the plant, what you are concerned about, and 
what you are trying to do to respond to the situation as the event progresses. It would be helpful if you verbalize your thoughts and actions as you address these situations.

Following each scenario there will be a short debriefing to recap what happened during the event. You may be asked questions about what you thought was happening, your concerns, and why you took the actions you did.

Based on the analysis of the recorded data, written descriptions of the events run and how they were handled will be generated. These descriptions will be used as input in developing and testing the simulation program. They may also be included in articles for professional society journals. Every reasonable effort will be made to preserve the anonymity of the crew participants. The reports will not mention the plant at which the exercises were run.

Are there any questions? (Answer questions)

If there are no more questions, before we start, I would like to ask whether any of you have heard anything about the accident scenarios that we are using in the study. Without telling me what you think the event is, can each of you answer "Yes, I have," or "No, I have not heard what the accident scenario is, or any other information that might help an operator respond to the event?"

[Ask each operator individually to say whether they have heard what the events are. If anyone says yes they have heard pertinent information, take them aside and have them tell one of the instructors what they have heard. Without naming particular individuals who might have told them about the experiment, ask them to describe in general how they found out about it. If the accident scenarios described by the operator are the correct ones, then we might have to cancel the session. If his/her description of the scenarios is incorrect or so vague as to encompass many potential scenarios, we can let him/her know that these are not the events we will be running, and ask if he/she is still interested in participating.]

[After this ask: "Is there anything else about the experiment you have heard that you want to mention?"]

\section{At the end of the two scenarios the participating operators will be told:}

Thank you for participating in the research. The exercises you have just participated in will be very useful to us in developing a computer model of how crews handle challenging scenarios. Hopefully this computer model will help us provide improved means to support you in doing your job.

We will be running these same accident scenarios with other crews of operators at this plant and possibly also at other plants. We want to make sure that operators who might participate in the research do not find out ahead of time any information about the scenarios or how they might be handled. As a result we ask you to please not discuss your experience in the research, and especially the scenarios we ran, with others who have not yet participated in the research. Your cooperation in keeping these scenarios confidential is critical to ensure that the results of the research are meaningful and helpful.

Thank you again for your cooperation. 


\section{Sample Summary Sheet:} ISLOCA Case

CREW

DATE

$\begin{array}{lll}\text { Years } & \text { Activities } & \text { Other } \\ \text { Licensed } & \text { In Last Year } & \text { Exp/Educ. }\end{array}$

SS

so

RO

BOP

STA

\section{ISLOCA}

1. Did any crew member have prior knowledge of the event? $\mathrm{Y} / \mathrm{N}$ Which position?

2. Did event sequence go as planned? $\mathrm{Y} / \mathrm{N}$

3. Did the operators:

a. Identify RHR disturbance? $\mathrm{Y} / \mathrm{N}$

b. Connect RHR/PRT symptoms? $Y / N$

c. Connect RHR/AUX BLDG symptoms? $\mathrm{Y} / \mathrm{N}$

d. Connect RHR/CTMT symptoms? Y/N

e. Attempt to close RHR valves? $Y / N$

f. Stay in LOCA procedure? $\mathrm{Y} / \mathrm{N}$

g. Transition/Use LOCA Outside Containment procedure? $\mathrm{Y} / \mathrm{N}$

h. Transition/Use Loss of Emergency Coolant Recirculation procedure? Y/N

REMARKS: 
Sample Summary Shett

Sample Summary Sheet:

Loss of Heat Sink Case

CREW

DATE

Years

Activities

Other

Licensed

In Last Year

Exp/Educ.

SS

so

RO

BOP

STA

\section{LOSS OF HEAT SINK}

1. Did any crew member have prior knowledge of the event? $\mathrm{Y} / \mathrm{N}$ Which Position?

2. Did event sequence go as planned? $\mathrm{Y} / \mathrm{N}$

3. Did the operators:
a. Identify abnormal PZR Pressure? $\mathrm{Y} / \mathrm{N}$
b. Identify leaking PZR PORV? $\mathrm{Y} / \mathrm{N}$
c. Activate SI? $\mathrm{Y} / \mathrm{N}$
d. Correctly manage Loss of Heat Sink? $\mathrm{Y} / \mathrm{N}$
e. Bleed and Feed? $\mathrm{Y} / \mathrm{N}$

REMARKS: 


\section{Appendix C: Behaviorally Anchored Rating Scales (BARS)}

\section{Introduction and Source of BARS}

Attached àre copies of the draft Behaviorally Anchored Rating Scales (BARS) of team interaction skills developed by Montgomery et al. (1992) under the sponsorship of the U. S. Nuclear Regulatory Commission. These rating scales are taken directly from a draft report by Montgomery et al. (1992). The BARS scales were used in this study to rate crew interaction skills in the simulated scenarios. 


\section{GUIDELINES FOR MAKING BEHAVIORALLY ANCHORED RATINGS}

General Guidelines

- Consider how crew members interact.

- Focus on the crew as a team.

- Avoid making ratings based on a single crew member.

Facts about the Behavioral Frequency Ratings Scales

- A 7-point scale is used.

- For these ratings you will consider how cpen behakes on a given dimension.

\section{Guidelines for Making Ratings.}

- Carefully read the anchors for each tear inferaction skills dimension.

- If the crew always behaved in the manner bescribed in the statement, then circle the number above that rating.

- If the crew did not behore in the-exact maniter described in the anchor, circle the mos appeapriate number between the two anchors that best describe the crew' beliovior. Remember, for each dimension a number on the scale must be cijcigo.

Example of the 7-Point Behayiorally Anchoked Rating Scale

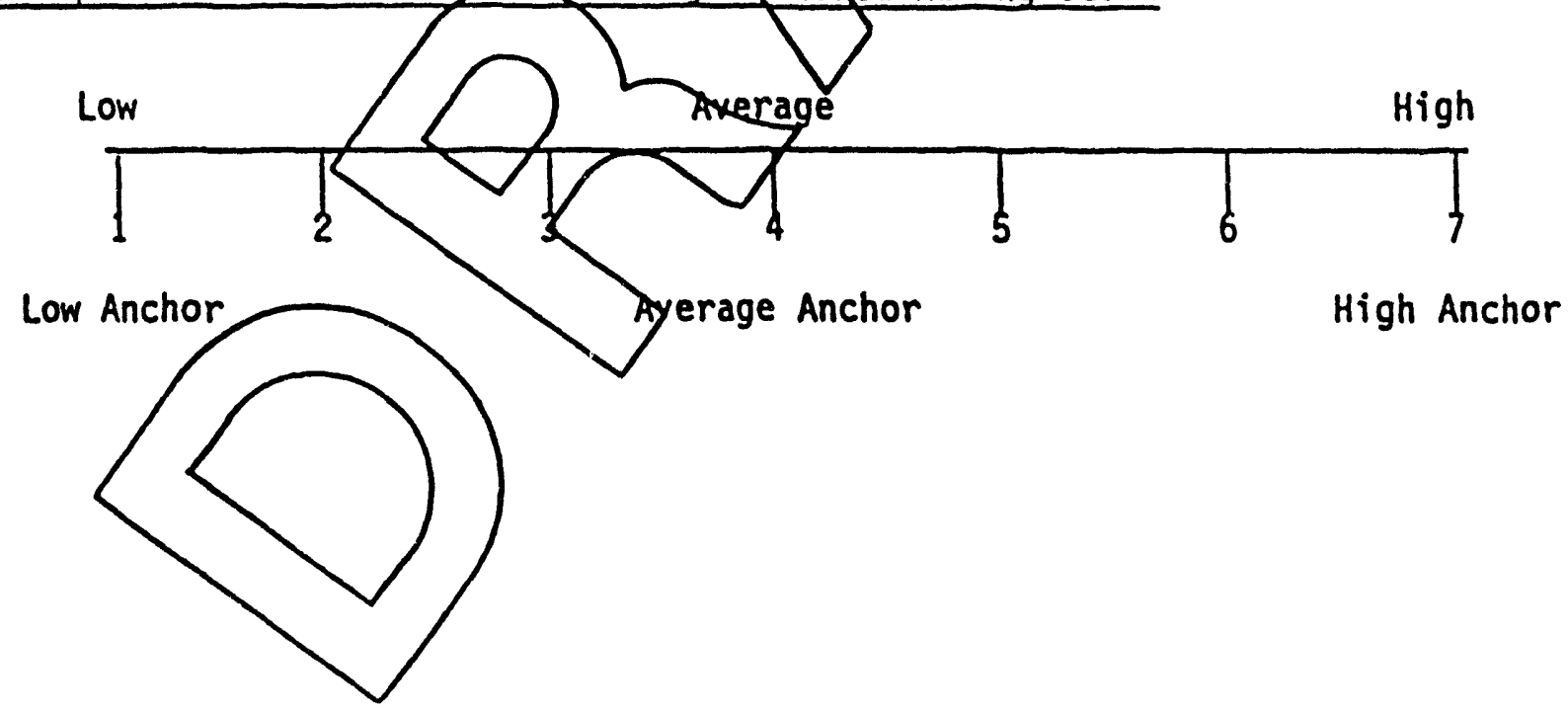


BARS -- COMMUNICATIONS

LOW

AVERAGE

HIGH

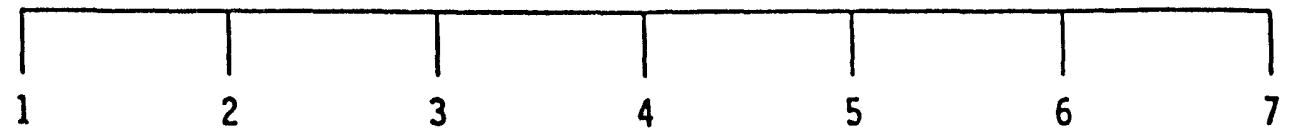

LoW

Provide insufficient

information about plant status and plans for stabilizing the plant. Crew members are difficult to understand when transmitting factual information. They seldom acknowledge the receipt of factsa? information. Communications include a high proportion of nontask-relevant information.

\begin{tabular}{|c|c|}
\hline & \\
\hline 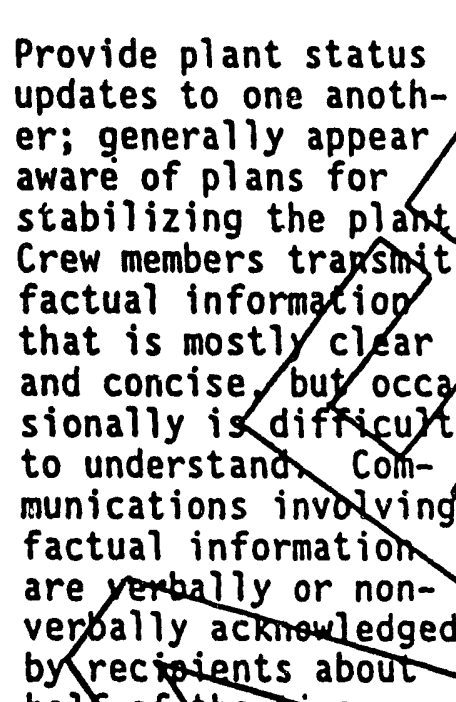 & $\begin{array}{l}\text { Maintain constant } \\
\text { awareness of plant } \\
\text { tatus and plans for } \\
\text { stakilizing the plant. } \\
\text { crew members transmit } \\
\text { factual information in } \\
\text { a cleez Ind concise } \\
\text { manner Communica- } \\
\text { tions including fac- } \\
\text { tual information are } \\
\text { always verbally or } \\
\text { nonverbally acknowl- } \\
\text { edged by recipients } \\
\text { (e.g., "I understand," } \\
\text { or waving a hand or } \\
\text { making the "OK" sign). }\end{array}$ \\
\hline
\end{tabular}

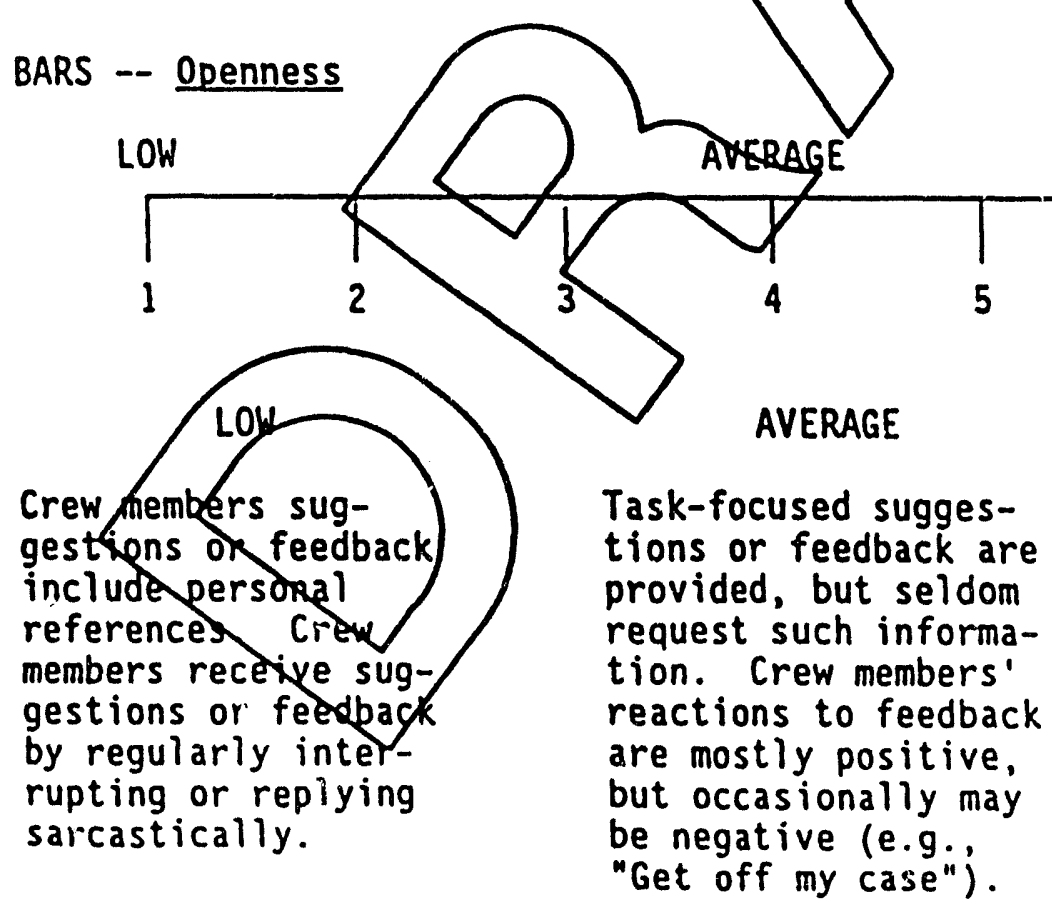


Behaviorally Anchored Rating Scales

BARS -- TASK COORDINATION

LOW

AVERAGE

$\mathrm{HIGH}$

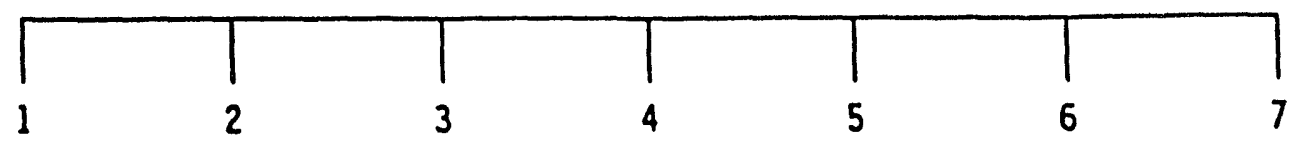

LOW

AVERAGE

Resources within the control room are allocated without considering the task. Consult procedures, but do not rely on them to guide responses.

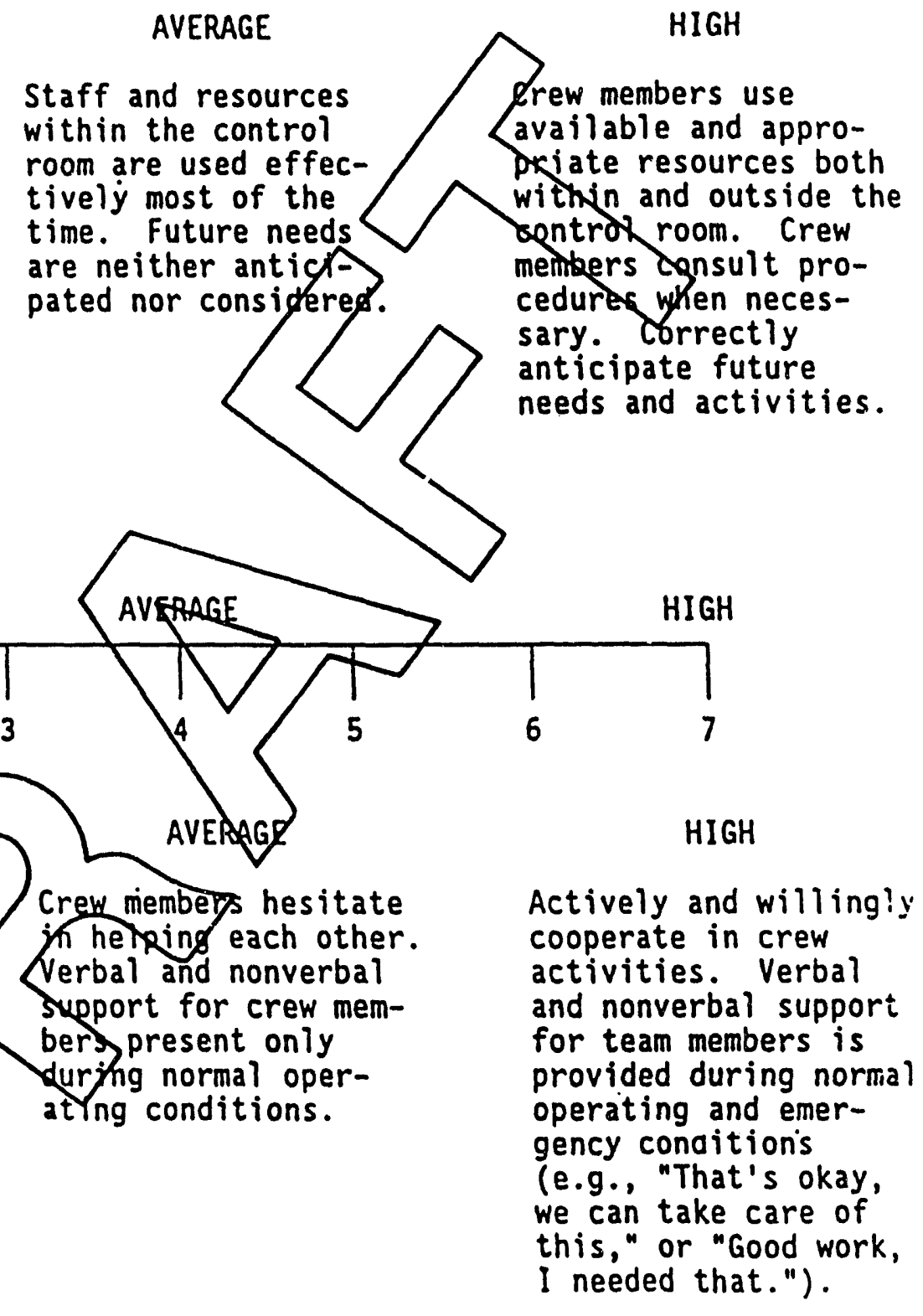


BARS -- MAINTAINING TASK FOCUS IN TRANSITIONS

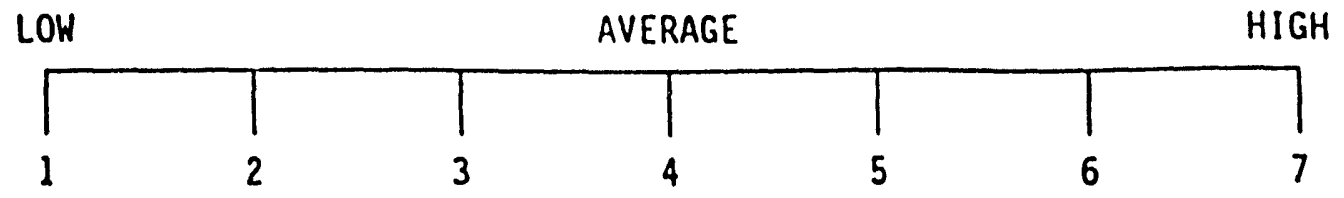

LOW

Crew members express anger or frustration to each other when novel or unusual conditions occur.

BARS -- ADAPTABILITY

AVERAGE

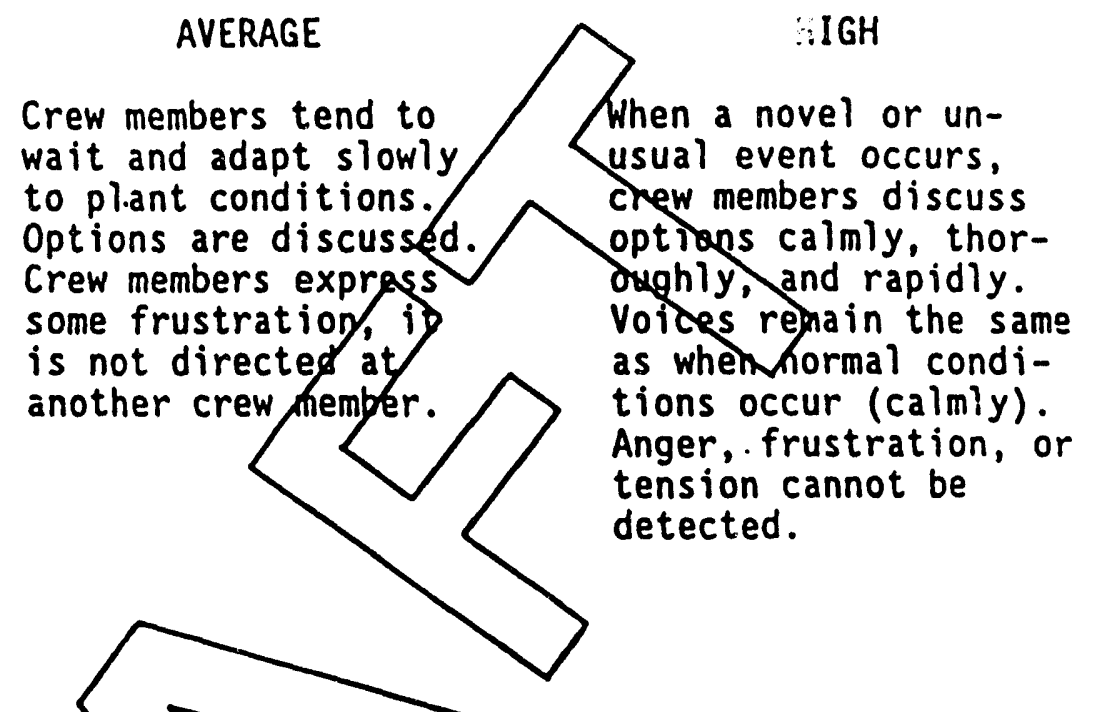

LOW

HIGH

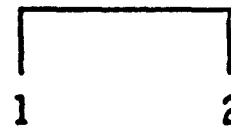

LOW

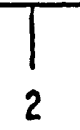

$2^{3}$

After a change in plant conditions, crew members occasionaty recognize/the need to change, prigrities may or may/not/change, abd some work ass ignments eventalily change, bytt others
After a change in plant conditions, crew memgers may recognize

tof need to change, often change priorities slowiy, and change work assignments only after a significant period of time elapses.
HIGH

After a change in plant conditions, crew. members immediately recognize the need for change and rapidly shifts priorities to reflect changing and rapid adjustments in work assignments. 


\section{Appendix D: A Cognitive Demands Checklist}

This project has provided evidence for the role of cognitive activities in guiding operator performance in complex accident scenarios. We developed a Cognitive Demands Checklist that is intended to capture some of the findings of the project in a form that can be used directly by the NRC staff to assess characteristics of an accident sequence or situation (e.g., characteristics of the event, the procedures, or the man-machine interface) that make errors of intention more likely.

The checklist provides a structured list of factors (e.g., characteristics of the event, the procedures, the manmachine interface) that can result in errors of intention (deciding to take a wrong action). It also includes factors that can contribute to errors of execution (intending to take the correct action but executing it incorrectly). The structured list is guided by the model of cognitive performance that underlies the CES simulation, and the results of the empirical analysis of crew performance in simulated emergencies. The results of both the CES simulation efforts and the empirical study of crew performance emphasize the importance of situation assessment and the expectations derived from this situation assessment in the formation of operator intention. The checklist also draws on the Rasmussen model of operator performance (Rasmussen, 1986) as well as other cognitive psychology literature on decision processes and decision biases (Kahneman, Slovic and Tversky, 1982) and error classification schemes (Norman, 1981; Reason, 1990).

The list is prima rily targeted at operator performance during emergency situations where performance is guided by Emergency Operating Procedures. It contair.s a list of factors that are likely to help performance as well as factors that are likely to hinder performance.

The Cognitive Demands Checklist can be used by NRC staff members as a "check list" to identify situations that can lead to cognitive errors. The checklist can be used:

(1) to establish a protocol for use by an onsite incident investigation team;

(2) to identify common psychological root causes across different incidents;

(3) to investigate potential cognitive sources of error as part of a human reliability analysis;

(4) to design/classify/calibrate accident scenarios used in simulator training and testing with respect to cognitive (i.e., "thinking.") skills being exercised and level of difficulty;

(5) to evaluate the potential impact of proposed changes in M-MI, training, or procedures on cognitive performance.

The Cognitive Demands Checklist can be incorporated as part of HRA analyses that use more traditional approaches for quantification of probability estimates such as THERP (Swain and Guttmann, 1983) or the Human Cognitive Reliability (HCR) model (Spurgin, Moieni, Gaddy, Parry, Orvis, Spurgin, Joksimovich, Gaver, and Hannaman, 1990). The checklist can be used as a screening tool to identify situations that may lead to cognitive error. These can then be analyzed in more depth, for example, by running crews through the events using training simulators as recommended by the HCR model, or by using expert judgment techniques such as SLIMMAUD. A similar approach has been recommended by Beare, Gaddy, Parry, and Singh (1991) as an adjunct to the HCR model. 


\section{Cognitive Demands Checklist}

\section{Detection/Observation}

Will the operator detect abnormal plant indications?

Help: Symptoms salient/alarmed

indicators are alarmed

parameter is highly salient (i.e., position; size; discriminability)

target and upper and lower bound values for parameter are displayed

other indicators are cuiet (no other alarms) when indications occur

operator workload is low when indications occur

Help: Operator has reason to check parameter

_ parameter is routinely monitored

procedures direct operator to monitor this parameter

hypotheses currently entertained suggest relevance of monitoring parameter

Hincler: Symptoms not salient

indicators are not alarmed

indicators are not located near likely operator positions (e.g., located on a back panel or outside the control room)

indicators are difficult to read out

other alarms occur at the same time

operator workload is high when indications occur

Hinder: Operator has no reason to check parameter

parameter is not routinely monitored

procedures do not direct operator to monitor this parameter

parameter not relevant to hypotheses currently entertained

\section{Hinder: Symptoms/indications are masked or obscured}

misleading indications exist (e.g., sensor failure; M-MI displays demand position rather than actual position) other malfunctions occur to obscure or mask primary event

other manual or automatic system action occurs to obscure or mask primary indications (e.g., shrink and swell)

symptoms may not yet be present or may have dissipated at point in procedure where request to monitor parameter is made 
Hinder: Identifying indicator value as abnormal requires mental effort (e.g., memory recall , mental calculations)

target and upper and lower bound values for parameter are not displayed mental calculation required (e.g., comparison of several indicators; calculation of rate) knowledge of special context required (e.g., setpoint shift)

\section{Situation Assessment - Explanation of Observed Plant Behavior}

Will the operator develop the correct interpretation of plant state?

Help: Explanation will be called to mind as a function of familiarity (frequency); recency; perceived likelihood; and representativeness of symptoms

symptoms/indications are very clear and lead to single conclusion

event is highly familiar to operators (e.g., frequently practiced on simulator; occurs with high frequency)

similar event has occurred recently or has otherwise been brought to the attention of the operators event is perceived by operators to be a high-likelihood event multiple symptoms/indications point to conclusion (e.g., valve position; flow rate; discharge temperature) procedural guidance for correct situation assessment is available the procedure has "catch" steps to detect errors in interpretation

Hinder: Other highly salient explanation is available that can account for much of the symptoms

_ symptoms can be (at least partially) explained by other known or hypothesized influences:

- a manual or automatic control system action (e.g., shrink and swell resulting from cooldown)

- another malfunction known to be present

_ symptoms can be (at least partly) explained by a more familiar hypothesis (e.g., events that are routinely practiced during training)

_ symptoms can be (at least partly) explained by an event that has recently occurred or has otherwise been brought to the operators' attention.

symptoms/indications can be "explained away" as "noise" or a false alarm.

__ symptoms appear in multiple diverse systems and require knowledge of system inter-connections to integrate into a coherent explanation

some critical indicator is available only to a single operator and is unlikely to be picked up by other control room personnel

event is perceived by operators to be a very low-likelihood event

cues are not reliable (given the event) 


\section{Intention to Act - Procedure Selection}

Will the operator identify and transfer to correct procedure?

Help: Indications and procedure criteria are clear for transition to correct procedure

criterion for transition to correct procedure is explicit step in current procedure or part of standard operating procedure

criterion for transition to correct procedure requires simple reading of indications and requires no judgment

or interpretation

Hinder: Indications may not be clear or criteria for transition may be ambiguous

criterion for transition to correct procedure is not explicit in current procedure

criterion for transition to correct procedure requires judgment or interpretation

criterion for transition to correct procedure requires sustained monitoring to judge (e.g., trends over time) primary indications for transition may not be manifest when transition step is reached primary indications for transition may dissipate or disappear before transition step is reached other indications may result in transition to another procedure before "desired" transition step is reached 58 there are strong indications to transfer to another procedure

\section{Intention to Act - Schedulino/Prioritizing the Action}

Are there factors that would cause an operator to postpone an action due to workload/scheduling constraints or cause him/her to forget to take the action (i.e., a memory lapse)?

Help: Action takes precedence over other actions and can be executed immediately

the action is very high in priority

the action can be executed immediately; it does not depend on completion of some other action or event the action is needed to allow other operators to continue working

\section{Hinder: Other actions compete for resources or there is delay before action can occur}

there are other actions of greater importance or greater urgency

the procedure is written to allow significant flexibility for sequencing of actions (e.g., words such as "as time permits...")

the action cannot be executed immediately because there is a need for another criterion to be satisfied first (e.g., wait till a parameter reaches value $x$ )

the action requires several operators to coordinate activities 58 This can arise in cases where there are multiple faults and/or where the initial fault produces secondary failures as a side effect (e.g., an
interfacing system loss of coolant accident leading to rupturing of the PRT and radiation in containment). 


\section{Intention to Act-Contributors to Intentional Deviation from Procedure}

Are there factors that would cause the operator to delay or avoid taking an action explicitly indicated in a procedure or to take an action outside procedures (i.e., commission errors)?

Help: Action will be taken in accordance with procedure: Action is compatible with all goals

the criteria for taking the action are clear and unambiguous

the action's effect is clearly understood and fits well with the goals of the current procedure

taking the action has no perceived negative consequences (i.e., no goal trade-offs)

In cases where there are multiple conflicting goals, the procedure provides clear guidance on goal prioritization (e.g., goal prioritization via status trees)

training and organizational climate (i.e., safety culture) instill and

reinforce appropriate goal prioritization

Hinder: Other goals conflict with action providing motivation to significantly delay or totally avoid action

taking action may violate standard operating practice (e.g., take operator out of usual operating band) 59

taking action may lead to reduced availability of safety systems, equipment, or instruments

taking action may have a potential negative effect on some other safety function (e.g., lead to overfill of pressurizer)

there is significant uncertainty or unknown risk associated with taking the action

(e.g., PORV after being opened may stick open)

taking the action will adversely affect areas within plant and further burden recovery (e.g., contaminate

Auxiliary Building which will increase effort needed to do maintenance)

taking the action will have severe consequences associated with cost (e.g., plant will be shut down for major cleanup after bleed and feed)

taking the action will release radiation to environment

Hinder: Consequences of delay (or omission) of action are perceived to be small

perception that action is not relevant or constitutes "overkill" under the particular circumstances perception that undesirable action can be delayed without negative consequences (i.e., with negligible probability of negative consequences)

criterion for taking action is perceived to be overly conservative process can be monitored and action taken if situation degrades

- delaying action would buy needed time to rectify situation by alternative means

action violated routinely without negative safety consequences (probability of negative safety consequences from failure to take action is extremely small)

\section{Hinder: Criteria for taking action are ambiguous}

criteria for taking action are ambiguous, difficult to determine, or require a judgment call requirement for action is presented in a caution

\footnotetext{
59 Conversely, an action that is outside procedures may be taken (e.g., blocking a safety system) if it is permitted or routinely performed under other circumstances without incurring negative consequences.
} 


\section{Execution}

Will the operator omit a step or execute it incorrectly?

Help: Context, procedures, etc. lead to specific actions

procedure is highly practiced or memorized

action is logically required to proceed in procedure (e.g., interlock or permissive)

controls are labeled or grouped to make them easily identified

execution uses controls with only two settings; controls are clearly marked

Hinder: Procedures incomplete, complex, or poorly formatted

procedure steps are not arranged in logical units (i.e., no higher order grouping)

procedure step contains complex logic that can be misinterpreted

procedure step includes negatives (e.g., "not")

procedure step includes complex conjunctions (e.g., "and" and "or")

action is presented in a caution or note (not in procedure step)

procedure is incomplete or underspecified (i.e., some necessary actions are not explicitly stated)

specific information (e.g., valve control number) is not specified in procedure

execution requires a long list of substeps

order of actions specified in procedure is inefficient (e.g., requires moving back and forth across control board) so that execution is likely to be done in order different from the order specified in the procedure. execution requires the use of more than one operating procedure

Hinder: Displays or controls lead to confusion (i.e., slip or "mode" error)

controls are not placed near important indicators that determine execution

controls are likely to be confused with other similar controls

controls go against standard operational stereotype (e.g., flip a toggle up to turn off)

control system has more than one setting, so that the same control action has different consequences

depending on the setting (i.e., a mode error; this occurs most commonly with soft-controls on computer display systems)

execution requires a control action to be taken outside the control room

a major component or set of actions is strongly associated with another context and

may, therefore, lead to inappropriate actions (capture-type slip)

Hinder: Difficult timing or coordination requirements

execution requires some type of continuous control (e.g., tuning) where feedback is difficult to judge (e.g., delayed in time)

execution requires maintaining a parameter within a tight operating band (e.g., to avoid inadvertent trip or safety system activation)

execution requires rapid response (e.g., a rapid rate of change that requires a quick response)

execution requires a difficult coordination between operators 
Behaviorally Anchored Rating Scales

\section{Z. Execution - Detection of Errore}

Will the operator recognize that an error has been made?

Help: Formal checks to identify errors

procedure has explicit catch steps or verifications other operators are likely to do careful checking of performance

there is a salient indication when error is made or when action was successful (e.g., alarm, interlock)

Hinder: Little or no feedback/indication error was made

other operators are all occupied in some other activity and will not check performance there is poor feedback on effect of control action

\section{Execution - Recovery from Error}

Will the operator be able to recover from error?

Help: Formal procedure to recover

there is procedure written for recovery from error

Hinder: Little or no indication of how error has changed situation; recovery actions unclear incorrect execution cannot be recovered due to damage done recovery requires a set of actions different from the set of actions done incorrectly there are severe time constraints for executing recovery actions 

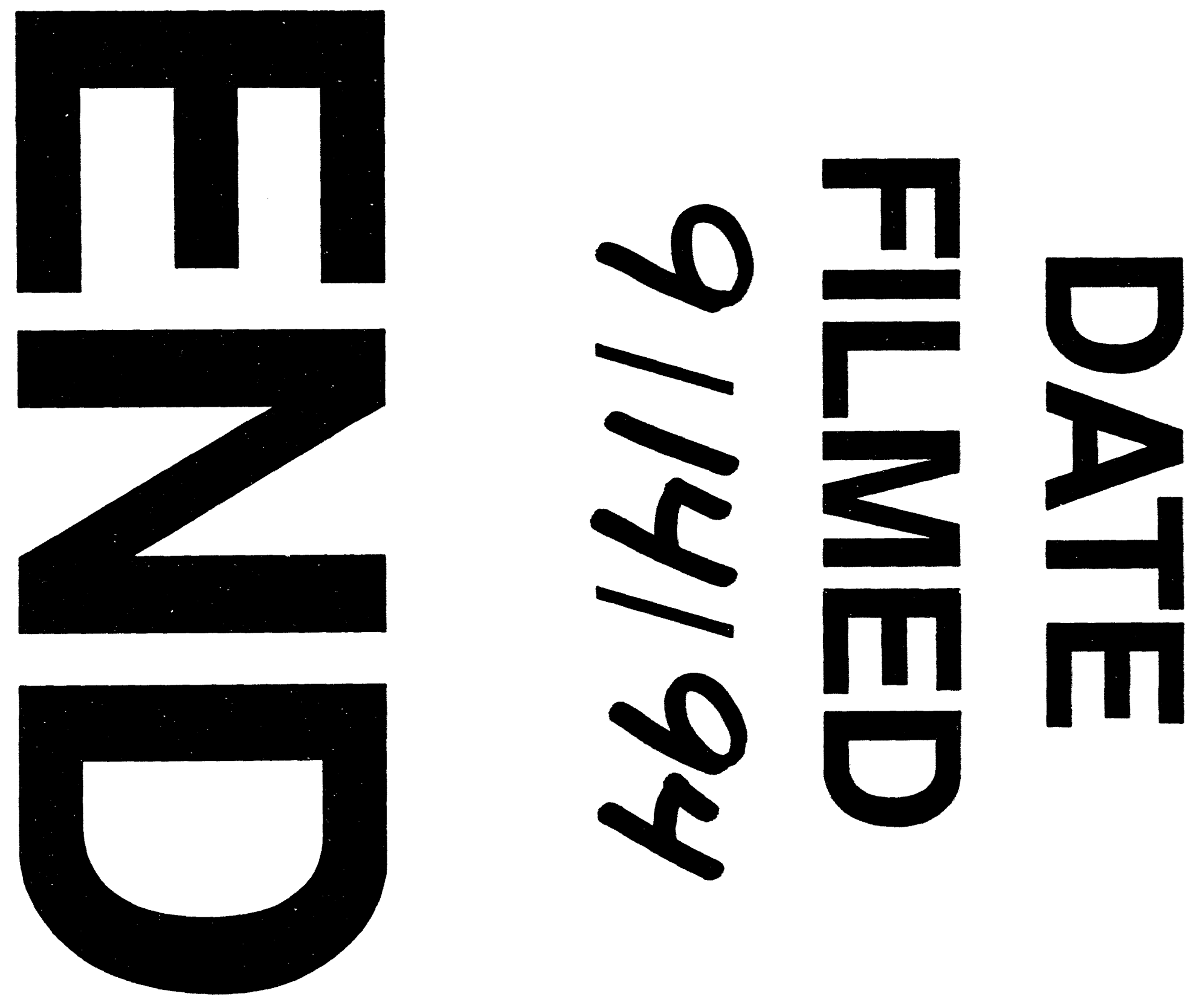
